\title{
Sportverletzungen des Kniegelenks
}

\author{
Meniskus - Bandapparat - Knorpel
}

Michael J. Strobel

\section{Zusammenfassung}

Das Kniegelenk ist sehr häufig bei Sportverletzungen betroffen. Neben Meniskusläsionen finden sich häufig Kapsel-Band-Läsionen mit Beteiligung der Seiten- und Kreuzbänder. Um eine möglichst optimale Wiederherstellung der sportlichen Aktivität zu gewährleisten, gilt es zunächst die Läsion exakt zu diagnostizieren. Anschließend ist eine differenzierte und abgestimmte Therapie erforderlich. Hierbei gilt es die konservativen Optionen gegen die operativen Möglichkeiten abzuwägen. Beim operativen Vorgehen müssen die verschiedenen Rekonstruktions- und Fixationstechniken gegeneinander abgewogen werden, um das optimalste und sicherste Vorgehen individuell für den jeweiligen Sportler zu wählen. Gleichzeitig muss den Anforderungen des Sportlers Rechnung getragen werden, möglichst schnell wieder mit seinem Sport beginnen zu können.

\section{Einleitung}

Das Kniegelenk ist sehr häufig von Sportverletzungen betroffen. Daher muss der sorgfältigen Diagnostik und Therapie besonderes Augenmerk geschenkt werden, um weitreichenden Spätschäden, Funktionseinschränkungen oder einem fortschreitenden Gelenkverschleiß vorzubeugen.

Zunächst gilt es eine definitive Diagnose zu stellen und dann zu entscheiden, ob eine konservative oder operative Therapie erfolgen soll. Im Falle der operativen Therapie gilt es festzulegen, ob diese so-

OP-JOURNAL 2008; 24: 96 - 134

(c) Georg Thieme Verlag KG Stuttgart • New York DOI 10.1055/s-2008-1038954

\section{Sports-Related Injuries of the Knee Joint}

The knee joint is very often affected by sports injuries. Besides lesions of the meniscus, lesions of the capsular ligaments involving both the lateral and the cruciate ligaments are very common. In order to achieve an as optimal as possible restoration of sports activities, it is at first necessary to exactly diagnose the damage. This is followed by a differentiated and individually adapted therapy. The pros and cons of both conservative options and surgical interventions must be carefully considered. In the case of surgical treatment again the various reconstruction and fixation techniques must be assessed and that procedure that is optimal and safest for the individual sportsman/-woman chosen. At the same time the requirement of the athlete to be able to take up his/her as sports activity as quickly as possible must be taken into consideration.
Ursache

Meniskusläsionen können durch banale Bewegungen wie Stolpern, Ausgleiten oder Sturz auf das Kniegelenk, aber auch durch forcierte Rotationsbewegungen, wie sie bei zahlreichen Sportarten vorkommen, verursacht werden. Nicht selten finden sich degenerative Vorschädigungen in Abhängigkeit vom Alter des Sportlers, der vorliegenden Beinachse und sportartspezifischer Bewegungsabläufe.

\section{Symptomatik}

Die Symptomatik reicht von Schmerzen im betroffenen Gelenkspalt bis hin zu akut auftretenden Streck- und/oder Beugehemmungen. Eine Gelenksperre kann durch Einklemmung eines abgerissenen Meniskusanteiles z.B. eines Korbhenkels oder Meniskuslappens hervorgerufen werden.

Das Rupturausmaß ist dabei nicht proportional zum vorliegenden Streckdefizit, wie man leicht annehmen könnte. Eine Streckhemmung von 50-60 findet sich bei begrenzten Rupturen, z.B. einem hinteren Längsriss. Reißt der Meniskus weiter in Richtung des Vorderhorns, wird hierdurch das Streckdefizit reduziert. Ein Knie mit geringem Streckdefizit ist daher besonders sorgfältig zu untersuchen.

Im Folgenden werden die wichtigsten Verletzungsmuster abgehandelt und sowohl das diagnostische Vorgehen, das therapeutische Management als auch die konservativen und operativen Therapieoptionen beschrieben. Von den möglichen Operationstechniken werden die vom Autor bevorzugten und bewährten Vorgehensweisen beschrieben.

\section{Meniskus}

Meniskusverletzungen zählen zu den häufigsten Verletzungen.

\section{Diagnostik}

Die Meniskusdiagnostik ist beim akut verletzten Kniegelenk nicht immer einfach, da das Gelenk äußerst schmerzhaft sein kann. Die Diagnostik erfolgt mit den bekannten Meniskustests (Tab.1). Die Symptomauslösung bei den Tests basiert auf 2 Faktoren:

1. Druckschmerz im Gelenkspalt, der durch Kompression des betroffenen Meniskusteils verstärkt wird. 
Tab. 1 Meniskustests (mod. nach Strobel et al. 1995).

1. Druckschmerz im Gelenkspalt

2. Hyperflexion und Hyperextensionsschmerz

3. Steinman-I-Zeichen

4. Steinman-II-Zeichen

5. Payr-Zeichen

6. Böhler-Zeichen

7. Krömer-Zeichen

8. Bragard-Zeichen

9. Merke-Zeichen

10. McMurray-Zeichen

11. Fouche-Zeichen

12. Childress-Zeichen

13. Apley-Test

14. Cabot-Zeichen

15. Turner-Zeichen

16. Tschaklin-Zeichen

17. Finochietto-Zeichen

\section{Bewegung, die den abgerissenen} Meniskus zum Subluxieren bringt. Diese Subluxation wird vom Patienten bemerkt, ist auch vom Untersucher im Gelenkspalt tastbar oder hörbar. Bei stärkerer Flexion wird der Hinterhornbereich, in streckungsnahen Positionen der Vorderhornbereich untersucht.

Die wichtigsten Meniskuszeichen sind der Druckschmerz im Gelenkspalt sowie der Rotationsschmerz in stärkerer Beugung. Wird das Kniegelenk gebeugt und außenrotiert, dann anschließend gestreckt, kommt es bei einer Innenmeniskushinterhornläsion $\mathrm{zu}$ einem Schnapp-Phänomen (McMurray-Test).

Differenzialdiagnostisch gilt es verschiedene Verletzungen zu unterscheiden [82]. Schmerzen auf der Medialseite können bei einer Verletzung des medialen Seitenbands oder einer Patellaluxation auftreten. Besonders die Teilruptur des medialen Seitenbands ist von der Meniskusläsion abzugrenzen, da auch hierbei ein schmerzhaftes Streckdefizit auftreten kann. Bei der Teilruptur des medialen Seitenbands tritt dies aber erst einige Stunden nach der Verletzung, meist am Morgen danach auf. Der Untersucher findet ein nicht komplett streckbares, schmerzhaftes Kniegelenk. Für die Streckhemmung wird sehr häufig eine Meniskusläsion angeschuldigt. Die genaue Anamnese zeigt aber, dass der Sportler seine sportliche Aktivität nach dem Ereignis noch fortsetzen konnte und erst am Morgen die Symptomatik aufgetreten ist. Bei Meniskusläsionen tritt die Streckhemmung da- gegen sofort nach dem Unfallereignis auf.

Die Prüfung der medialen Aufklappbarkeit führt zur Schmerzverstärkung (Dehnungsschmerz des medialen Seitenbands). Häufig findet sich ein Druckschmerz über dem medialen Femurepikondylus (Ursprung des medialen Seitenbands). Da diese Verletzung häufig beim Skifahren auftritt, wird dieser Schmerzpunkt auch „Skipunkt“ genannt. Ein intraartikulärer Erguss liegt meist nicht vor.

\section{Bildgebende Diagnostik}

\section{Röntgen}

Die Röntgenuntersuchung ist bei jeder Meniskusverletzung obligat. Radiologische Veränderungen finden sich nicht. Besteht die Meniskusverletzung über mehrere Monate, können kleine zipflige Ausziehungen am Tibiaplateau oder Femurkondylus als radiologischer Hinweis auf eine Meniskusläsion zu finden sein (Rauber-Zeichen).

\section{MRT}

Mit der MRT lässt sich eine Meniskusläsion mit hoher Sicherheit ausschließen bzw. bestätigen. Ausgedehnte Rissformationen oder Korbhenkelläsionen sind klar darzustellen. Schwieriger wird die Situation bei intrameniskealen Veränderungen. Oft findet sich eine sichelförmige Aufhellung im Meniskusgewebe (Abb.1). Diese als Typ-II-Läsion bezeichnete Veränderung ist im Zentrum des Meniskus lokalisiert, und kann, auch wenn sie nicht bis zur Unter- oder seltener Oberfläche des Meniskus reicht, zu einer klinischen Symptomatik führen. Gerade bei Sportlern, die das Kniegelenk im Rahmen ihrer sportlichen Aktivität häufig maximal beugen und belasten müssen, kann eine ausgeprägte Symptomatik bestehen.

Bei der klinischen Untersuchung finden sich oft ein diffuser Druckschmerz und ein Schnappen im Gelenkspalt (positives McMurray-Zeichen). Findet sich in der MRT keine typische Meniskusläsion, wird das weitere Augenmerk häufig nicht mehr auf den Meniskus gerichtet, insbesondere dann nicht, wenn der Radiologe die Veränderung wohlmeinend als intrameniskeale Aufhellung ohne Rupturzeichen beschreibt. Bei Sportlern sollte bei diesen Veränderungen und vorhandener klinischer Symptomatik eine Arthroskopie erwogen werden (s. unten).

\section{Arthroskopie}

Die Arthroskopie ist die diagnostische Technik, mit der Meniskusläsionen am zuverlässigsten diagnostiziert und in gleicher Sitzung therapiert werden können. Zunächst gilt es normale von pathologischen Befunden zu unterscheiden. Finden sich die klassischen Rissformationen, wie ein Längsriss (inkomplett oder komplett), Lappenrisse oder Korbhenkelläsionen, ist die Diagnostik einfach (Abb. 2). Gleiches gilt für degenerative Veränderungen, die lokalisiert sein können, aber auch den gesamten Meniskuskörper umfassen können. Derartige Läsionen sind häufig im Hinterhornbereich lokalisiert (Abb. $\mathbf{3}$ ).

Schwieriger wird die Diagnostik, wenn der Meniskus inspektorisch intakt erscheint. Veränderungen sind dann nur durch die Palpation (Tasthaken) zu diagnostizieren. Dies gilt für intrameniskeale Vernarbungen und Auswalzungen. Letzteres findet sich oft auch bei Typ-IILäsionen. Problematisch kann die Situation dann werden, wenn palpatorisch keine intrameniskealen Veränderungen oder Rissformationen nachweisbar sind, trotzdem ein Meniskusschnappen bei der klinischen Untersuchung auslösbar ist. In diesem Fall wird die Meniskusbasis sorgfältig palpiert, um einen hypermobilen Meniskus auszuschließen.

Seltener finden sich bei Sportlern Horizontalrisse im Außenmeniskus. Wird eine derartige Läsion zufällig entdeckt und besteht keine klinische Symptomatik, sollte diese Veränderung belassen werden. Bei alten Läsionen mit instabilen Meniskusfragmenten erfolgt eine Resektion der instabilen Anteile (Abb.4). Nach der Diagnose erfolgt in gleicher Sitzung auch die definitive Therapie im Sinne einer Meniskusresektion und/oder -refixation.

\section{Therapeutisches Management}

Findet sich bei der klinischen Untersuchung eine typische Meniskussymptomatik, ist die Indikation zur Arthroskopie gegeben. Eine MRT ist bei eindeutiger klinischer Symptomatik nicht obligat. Trotzdem bietet die MRT einige Vorteile zur Beurteilung der knöchernen Strukturen, hier insbesondere zum Ausschluss von Knochenödemen (Bone Bruises, s. unten). 


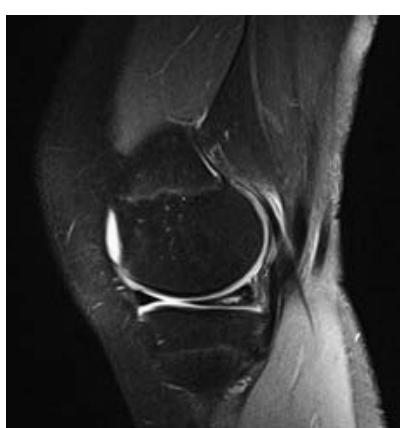

Abb. 1 Typ-II-Läsion am Innenmeniskushinterhorn be 16-jähriger Patientin (aktive Leichtathletin). Bei einer Resektion ist mit einer ausgedehnteren Meniskusentfernung zu rechnen, da sich die Läsion bis in das basisnahe Meniskusdrittel erstreckt.

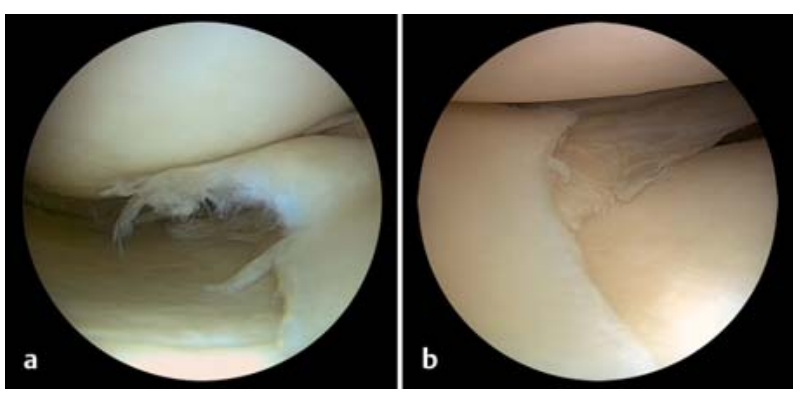

Abb. $4 \mathbf{a}$ und $\mathbf{b}$ Lappenriss des Innenmeniskus (a) und des Außenmeniskus bei 4 Jahre bestehender VKB-Insuffizienz (b).

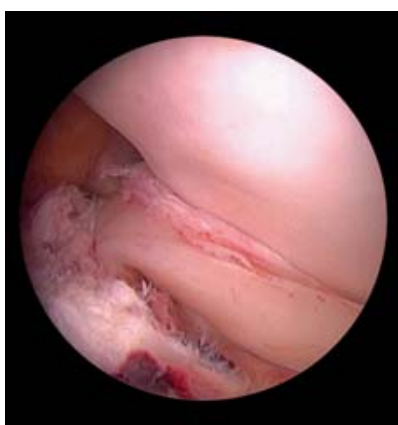

Abb. 2 Korbhenkelläsion am Außenmeniskus. Der abgelöste Meniskusteil ist in die Fossa intercondylaris eingeschlagen.

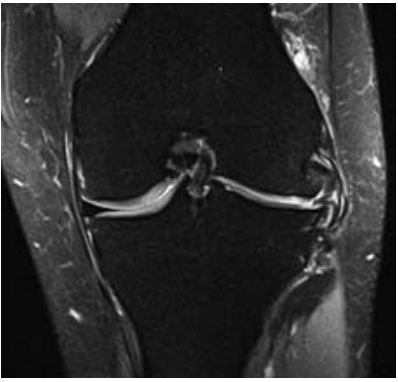

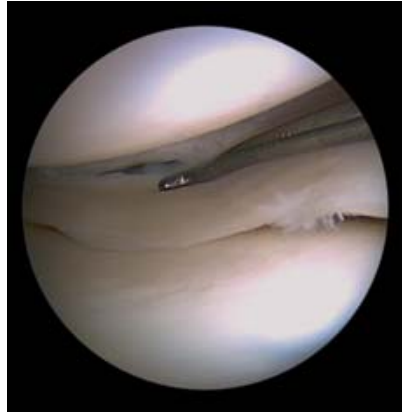

Abb. 3 Hinterer Längsriss am Innenmeniskus.
Bei einem positiven klinischen Meniskusbefund und einer unauffälligen MRT sollte trotzdem zur Arthroskopie geraten werden, da der klinischen Untersuchung und dem subjektiven Beschwerdebild die höhere Wertigkeit zuzuordnen ist.

Sind Berufssportler von einer Meniskusläsion betroffen, wird häufig in Absprache mit dem Trainer, dem Vereinsarzt und dem Manager das weitere therapeutische Vorgehen diskutiert. Nicht selten fühlt sich der Spieler trotz massiver klinischer Symptomatik verpflichtet, die Saison noch zu beenden. Vor diesem gut gemeinten Vorgehen muss gewarnt werden, insbesondere wenn eine signifikante klinische Symptomatik mit rezidivierender Ergussbildung vorliegt. Gerade chronische Ergüsse führen durch den hohen Gehalt an Zytokinen zur Verschlechterung bestehender Knorpelschäden.

Finden sich Meniskusläsionen bei jugendlichen Sportlern oder Kindern, muss zum operativen Vorgehen geraten werden, insbesondere wenn der Verdacht auf eine Außenmeniskusläsion besteht.

\section{Konservative Therapie}

Die konservative Therapie einer gesicherten Meniskusläsion bei bestehender klinischer Symptomatik ist nicht zu empfehlen. Sie erfolgt nur dann, wenn Kontraindikationen zum operativen Vorgehen vorliegen.

\section{Operative Therapie}

Bei einem Sportler, der seine Sportfähigkeit wiedererlangen will, wird die Indikation zum arthroskopischen Vorgehen eher großzügig gestellt, insbesondere wenn eine deutliche klinische Symptomatik, subjektive Beschwerden und auch ein positiver MRT-Befund vorliegen.

Präoperativ sind Begleitverletzungen wie Kapsel-Band-Verletzungen auszuschließen. Findet sich z. B. eine alte VKBLäsion, sollte mit dem Sportler unbedingt diskutiert werden, ob nicht in gleicher Sitzung mit der Meniskustherapie auch die Bandstabilisierung erfolgen soll. Bei einer Meniskusrefixation hat diese eine wesentlich bessere Prognose, wenn die Bandstabilisierung in gleicher Sitzung erfolgt.
Ist ein Berufssportler oder Hochleistungssportler betroffen, muss bei der Indikationsstellung zur Meniskusrefixation immer die folgende lange Nachbehandlungszeit und die damit bedingte lange Sportpause berücksichtigt werden (s. unten). Daher wird gerade bei Innenmeniskusläsionen die partielle Resektion favorisiert.

Die Indikation zum operativen Vorgehen ist besonders dann gegeben, wenn bei Kindern und Jugendlichen eine Außenmeniskusläsion vorliegt, da auch heute immer noch keine suffizienten therapeutischen Möglichkeiten bestehen, nach einer ausgedehnten Resektion am Außen- und/oder Innenmeniskus das Meniskusgewebe mit einem Transplantat zu ersetzen. Nach einer Meniskusresektion kann man sich sehr einfach an einer einfachen Faustregel orientieren. Werden $20 \%$ des Innenmeniskus reseziert, steigt der Druck im medialen Gelenkkompartment um 20\%. Werden 20\% vom Außenmeniskus reseziert, steigt der Druck im lateralen Kompartment dagegen um 40\% - also doppelt so stark wie auf der medialen Seite an. Dies unterstreicht die besondere Bedeutung des Außenmeniskus. 


\section{Operationstechnik}

Meniskusläsionen sind die häufigste Indikation zur Arthroskopie. Es bestehen verschiedene therapeutische Möglichkeiten.

1. Belassen der Läsion. Wird bei einer Arthroskopie z.B. ein Horizontalriss am Außenmeniskus zufällig entdeckt und besteht keine klinische Symptomatik, kann die Läsion belassen werden.

2. Anfrischen der Läsion. Rupturen, die kleiner als $1 \mathrm{~cm}$ sind, wie z.B. inkomplette Längsrisse, werden mit einer kleinen Raspel, einem Mikrofrakturmeißel, einer kleinen Kürette oder dem Shaver angefrischt.

3. Resektion. Die Meniskusresektion ist die häufigste arthroskopische Operation. Hierbei wird der instabile Meniskusanteil bzw. das abgerissene Meniskusfragment entfernt, bis eine stabile Restbasis verbleibt. Nach der Resektion wird diese mit dem Tasthaken palpiert. Zeigen sich hier instabile Anteile, müssen diese auch entfernt werden. Auf eine komplette Entfernung der Restbasis wird aber immer verzichtet. Eine besondere Problematik stellen alte komplette und inkomplette Längsrisse dar, die mit einer chronischen VKB-Läsion verbunden sind. Hier gerät man bei der Resektion des instabilen Meniskusteils in die Meniskusbasis, die häufig ausgedehnte horizontale Risskomponenten aufweist. Es ist dann nicht immer einfach zu entscheiden, wie viel Meniskusgewebe reseziert werden soll und wie viel belassen werden kann. Die Entscheidung hängt im Wesentlichen von den Begleitparametern (Alter des Patienten, Beinachse, Begleitläsionen, sportliche Aktivität etc.) ab, um das genaue Vorgehen hinsichtlich der Resektionstiefe $\mathrm{zu}$ bestimmen. Eine horizontale Risskomponente kann durchaus belassen werden, da diese eher selten zu Einklemmungen führt. Dies trifft insbesondere auf den Außenmeniskus zu.

Eine Resektion am Außenmeniskus muss besonders vorsichtig erfolgen. Da dieser sehr fleischig aufgebaut ist, führt ein sehr aggressives Vorgehen, z.B. mit dem Shaver, schnell zu einer ausgedehnten Meniskusresektion, die sehr leicht in das basisnahe Drittel reicht [83]. Dieser Zustand muss unbedingt vermieden werden. Daher sollten motorisierte Instrumente (Shaver) am Außenmeniskus sehr zurückhaltend eingesetzt werden. Siche- rer ist das Vorgehen mit einem ovalen Punch. Eine ausreichende Übersicht über den Außenmeniskus muss bei der Resektion immer gegeben sein. Ansonsten kann die Resektion sehr leicht zu ausgedehnt geraten oder sogar posterior des Meniskus gelegene Strukturen wie z.B. die Popliteussehne gefährden.

Lässt sich am Außenmeniskus eine ausgedehnte Resektion nicht vermeiden, muss der Sportler auch im Hinblick auf seine weitere sportliche Aktivität beraten werden. Handelt es sich nicht um einen Berufs- oder Hochleistungssportler, muss gegebenenfalls ein Wechsel der sportlichen Aktivität oder eine Reduktion diskutiert werden, da immer mit einem zunehmenden degenerativen Verschleiß im lateralen Gelenkbereich (laterale Kompartmentarthrose) zu rechnen ist (Abb. 5).

Bei einer Typ-II-Läsion ( Abb.1) kann der Meniskus völlig intakt aussehen. Findet sich eine intrameniskeale Aufweichung oft mit Auswalzung und lässt sich der Meniskus weit in das Gelenk hineinziehen, erfolgt bei bestehender klinischer Symptomatik eine partielle Meniskektomie. Hierbei wird der Meniskusrand gekürzt, sodass sich der Meniskus nicht mehr zwischen Femur und Tibiaplateau einklemmen kann. Alle Techniken der Refixation oder Anfrischung haben sich bei diesen Veränderungen nicht bewährt, insbesondere nicht bei Sportlern, bei denen die Beschwerden im Rahmen ihrer sportlichen Aktivität auftraten. Trotzdem muss man sich bewusst sein, dass in einigen Fällen die Resektion ausgedehnter wird als geplant, da die Veränderungen bis in das basisnahe Meniskusdrittel reichen können.

4. Refixation. Wenn möglich, sollte eine frische Meniskusläsion, insbesondere wenn sie ausgedehnt ist, refixiert werden. Es existiert kein besseres Gewebe, das den Meniskus ersetzen kann, wie das Meniskusgewebe selbst. Verschiedene Techniken stehen zur Verfügung.

Outside-in-Technik. Die einfachste und kostengünstigste, gleichzeitig auch stabilste Technik ist die Outside-in-Technik. Hierzu werden lediglich 3 normale Injektionskanülen und 2 PDS-Fäden der Stärke 2-0 benötigt. Eine Kanüle dient als Orientierungskanüle. In die beiden anderen Kanülen werden jeweils PDS-Fäden eingefädelt. Ein PDS-Faden wird mittels einer Klemme zu einer Schlaufe gelegt (Schlaufenkanüle). Bei der anderen Kanüle steht das Fadenende ca. $5 \mathrm{~mm}$ über die Kanülenspitze (Kanüle mit freiem Fadenende). Zuerst wird die Orientierungskanüle eingestochen, um die richtige Höhe für die Refixation auszuloten. Hat die Orientierungskanüle die Meniskusbasis und das abgerissene Meniskusteil passiert, wird die Kanüle mit dem freien Fadenende eingestochen. Direkt neben dem Einstich erfolgt eine sparsame Hautinzision. In diese wird die Kanüle mit der Fadenschlaufe vorgeschoben. Es sollte immer eine Vertikalnaht angestrebt werden, da sich hiermit eine bessere Adaptierung des abgelösten Meniskusfragments zur Meniskusbasis erreichen lässt. Alternativ bieten sich Horizontalnähte an, die jedoch abwechselnd supra- und inframeniskeal verlaufen sollten, um eine Dehiszenz der adaptierten Meniskusteile zu vermeiden.

Inside-out-Technik. Bei dieser Technik werden spezielle Nadeln mit Faden von innen durch das abgelöste Meniskusfragment und dann durch die Meniskusbasis nach außen vorgeschoben. Um eine Läsion der posterior verlaufenden Gefäß-Nerven-Stränge zu vermeiden, wird dorsomedial bzw. dorsolateral eine Hilfsinzision angelegt. Diese Technik findet nur eine sehr eingeschränkte Verwendung.

All-inside-Technik. Bei den All-inside-Techniken erfolgt die Naht rein intraartikulär. Zuerst wurden Pfeile (Meniskusdarts, Meniskus-Arrows) entwickelt. Diese Fixationsimplantate wurden durch das abgelöste Meniskusfragment in die Meniskusbasis eingebracht. Die Applikatoren waren jedoch teilweise sehr groß, dass schon hierdurch Knorpelschäden am betroffenen Femurkondylus entstehen konnten. Da der Meniskus eine mobile Struktur ist, kommt es unter Flexion und Extension zu ausgedehnten Meniskusbewegungen. Dies führte bei Vorliegen einer Bandinstabilität dazu, dass derartige Fixationsimplantate sich komplett aus dem Meniskus gelöst haben. Zudem bleibt zu berücksichtigen, dass Meniskusgewebe ein relativ bradytrophes Gewebe ist. Resorbierbare Implantate finden hier sehr ungünstige Umgebungsbedingungen. Daher ist von der Verwendung derartiger Implantate abzuraten.

Bei sehr weit posterior lokalisierten Meniskusläsionen, die an der Aufhängung im Hinterhornbereich lokalisiert 

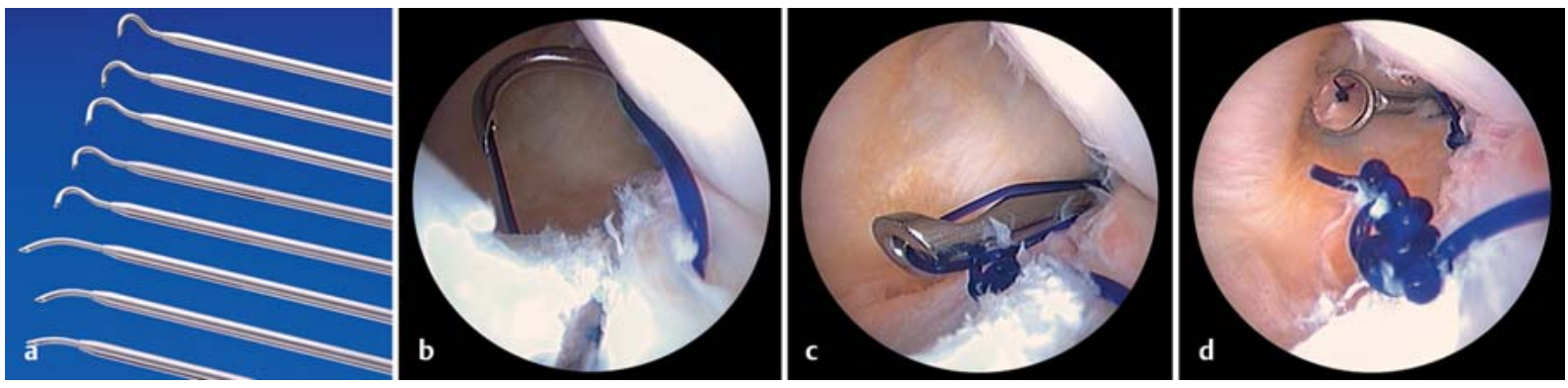

Abb. 6 a bis d All-inside-Naht einer Rampenläsion. Zur Naht sind verschieden geformte Nahthaken erforderlich (Fa. Karl Storz, Tuttlingen) (a). Platzieren der Naht über den dorsomedialen Zugang nach Anfrischen der Rupturzone (b) und Vorschieben des Knotens mit dem Knotenschieber (c). Kontrolle des Endzustandes mit dem Spiegel (d).
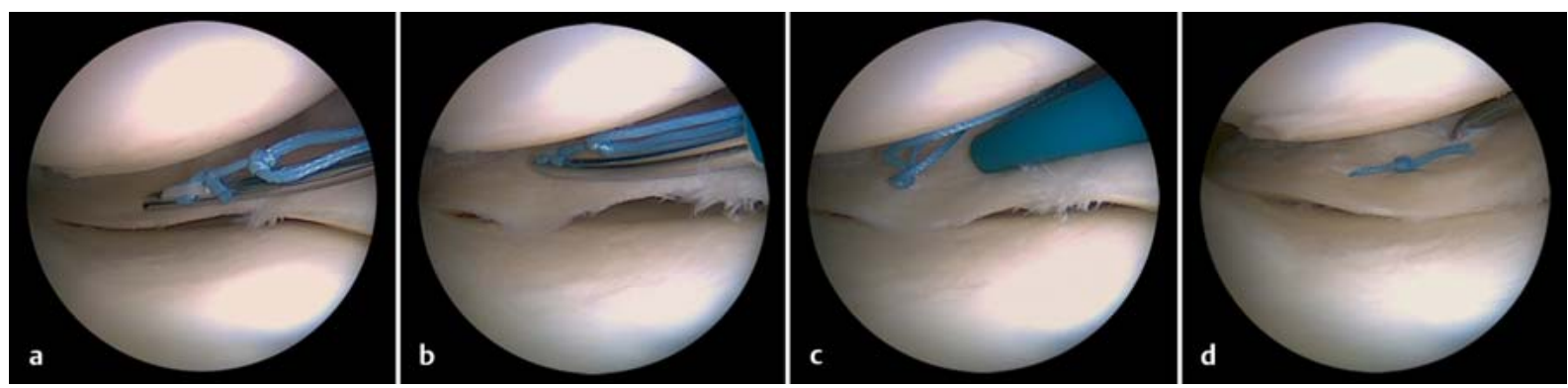

Abb. 7 a bis d All-inside-Naht mit Fast-Fix (Fa. Smith \& Nephew, Hamburg). Das Nahtinstrumentarium wird eingeführt (a) und in den abgerissenen Meniskusteil eingestochen (b), dann zurückgezogen und etwas weiter anterior superior erneut eingestochen (c). Nach dem Setzen der beiden Nahtanker, die mit einem speziellen Knoten verbunden sind, wird der Faden angezogen. Hierdurch zieht sich das Doppelankersystem zusammen und fixiert das abgerissene Meniskusfragment. Palpation nach Fixation (d).

sind, empfiehlt sich eine All-insideNahttechnik mit einem speziellen Nahtinstrumentarium (Abb. 6).

Bei posterioren Längsrissen oder Korbhenkelläsionen ist die Fixation im posterioren Meniskusdrittel mit einem Doppelankersystem, das mit einem speziellen vorgelegten Knoten in Verbindung steht (Fast-Fix, Fa. Smith \& Nephew, Hamburg) sehr einfach und schnell durchzuführen (Abb. 7).

Für die Meniskusrefixation im vorderen und mittleren Meniskusdrittel (Pars intermedia) hat sich die Outside-in-Technik bewährt. Im posterioren Meniskusdrittel (Hinterhorn) wird die All-insideTechnik z.B. mit der Fast-Fix-Technik oder einer All-inside-Naht mit einem speziellen Nahtinstrumentarium favorisiert (Abb. 6 und 7).

\section{Nachbehandlung}

Nach einer Meniskusresektion kann der Sportler voll belasten, wenn keine Schmerzen bestehen. Die Vollbelastung dient zudem auch als Thromboseprophylaxe. Postoperativ sind zirkuläre, nur das Knie umfassende Verbände zu vermeiden. Bei Schwellungen werden Lymphdrainagen rezeptiert. Sportfähigkeit ist in Abhängigkeit der Sportart, des Leistungsniveaus und der Ausdehnung der Meniskusresektion zwischen der 3. und 6. postoperativen Woche wieder möglich.

Nicht selten nehmen Sportler, insbesondere wenn es sich um Berufssportler handelt, die Trainingsaktivitäten sehr früh wieder auf, insbesondere wenn keine Schmerzen vorhanden sind. Hierbei ist der Zustand des Gelenks nach den Trainingseinheiten bzw. nach der Belastung zu kontrollieren. Treten z.B. 1-2 Wochen postoperativ Schmerzen auf, muss an eine Überbelastungsreaktion gedacht werden. Die Schmerzcharakteristik unterscheidet sich meist von derjenigen der Meniskusläsion. In diesem Fall ist zu einer MRT-Untersuchung zu raten. Nicht selten findet sich ein mehr oder minder ausgedehntes Knochenödem (Bone Bruise). In dieser Situation ist die Belastungsintensität zu reduzieren, bis sich das Knochenödem zurückgebildet hat und das klinische Beschwerdebild abgeklungen ist.
Nach einer Refixation wird in Abhängigkeit von Begleitverletzungen und Lokalisation der Refixation (Innen- oder Außenmeniskus) vorgegangen. Im Allgemeinen ist eine Schienenbehandlung von 4 bis 6 Wochen unter axialer Belastung angezeigt. Die Patienten dürfen mehrmals am Tag die Schiene abnehmen und Pendelbewegungen durchführen (maximal $90^{\circ}$ Flexion). Unbedingt sind Beugung und gleichzeitige axiale Belastung zu vermeiden. Mit derartigen Belastungen darf erst nach 6 bis 8 Wochen begonnen werden. Die tiefe belastende Hocke sollte für mindestens 3 Monate nicht erfolgen.

Beim Sportler ist mit einer Sportpause von mindestens 8-12 Wochen zu rechnen. Besonders vorsichtig ist vorzugehen, wenn es sich um eine Refixation eines Außenmeniskuskorbhenkels bei Jugendlichen und Kindern handelt. Hier ist eine Sportpause von mind. 6 Monaten zu empfehlen. 


\section{Vorderes Kreuzband}

Läsionen des vorderen Kreuzbands (VKB) sind die häufigste Bandverletzung des Kniegelenks [30,44,53,82]. In Anbetracht der steigenden sportlichen Aktivität, auch im Breitensportbereich, nehmen Diagnostik, Therapie und Rehabilitation einen großen Stellenwert auch in sozioökonomischer Hinsicht ein [71].

\section{Ursache}

Rotationstraumen, eine forcierte Flexion-Valgus-Außenrotation oder Flexion-Varus-Innenrotation aber auch Hyperextensionsmechanismen oder reine Valgustraumata können zu einer VKBLäsion führen [52,59]. Manchmal sind aber auch Bagatelltraumata wie ein plötzliches Hängenbleiben mit dem Fuß oder eine schnelle Richtungsänderung verantwortlich. Sehr häufig sind Verletzungsmechanismen mit einem Richtungswechsel, wie sie z.B. beim Fußball und bei vielen anderen Ballsportarten, aber auch beim Skifahren vorkommen, verbunden.

Frauen sind, im Vergleich zu Männern, bei manchen Sportarten (Fußball, Basketball, Volleyball) besonders verletzungsanfällig, da sie eine größere Knielaxität und eine allgemeine Bandlaxität aufweisen. Wie Myar et al. [54] nachweisen konnten, sprechen die erhöhte passive anteroposteriore Schubladenbewegung und eine passive Hyperextension des Kniegelenks für ein erhöhtes Verletzungsrisiko des VKB.

\section{Symptomatik}

\section{Akute Läsion}

Bei einer akuten Verletzung wird über einen heftigen Schmerz, oft auch über ein Krachen berichtet, das Gelenk schwillt kurz nach der Verletzung an $[52,82]$. Ein derartig schnell auftretendes Hämarthros ist typisch bei dieser Verletzung. Zudem kann der Patient seine sportliche Aktivität meist nicht weiter fortsetzen. Ein Hämarthros ist aber nicht obligat. Insbesondere bei komplexen Bandverletzungen kann das Blut durch die rupturierte Gelenkkapsel in die peripheren oder hinteren Weichteile austreten.

\section{Chronische VKB-Läsion}

Bei chronischen VKB-Läsionen stehen die Folgen der resultierenden Patho- mechanik durch den Verlust des RollGleit-Mechanismus im Vordergrund. Hieraus entstehen exzessive anteriore Tibiabewegungen in Relation zum Femur. Dies führt zur vermehrten Belastung des Knorpels, der Menisken - hier insbesondere der Hinterhornbereiche aber auch der peripheren Kapsel-BandStrukturen [52,72].

Die Patienten klagen über Schmerzen und/oder Unsicherheitsgefühl. Das Instabilitätsgefühl äußert sich im Wegknicken beim Richtungswechsel z.B. beim Aussteigen aus dem Auto oder auf unebenen Böden. Sportliche Aktivitäten können, da hierbei eine bessere neuromuskuläre Kontrolle vorliegt, manchmal noch ohne signifikante Beschwerden ausgeübt werden. Bisweilen stehen Meniskusbeschwerden, rezidivierende Gelenkschwellungen (chronische Synovitis) und/oder intermittierende Blockaden im Vordergrund der Beschwerden. Sekundäre Veränderungen wie eine chronische Ergussneigung oder eine Baker-Zyste können die gesamte Symptomatik darstellen, ohne dass der Patient subjektiv über eine Instabilität klagt. Erst die differenzierte klinische Untersuchung mit gezielter Anamneseerhebung führt zur eindeutigen Klärung.

Jede anteriore Tibiabewegung wird durch die Meniskushinterhörner gebremst. Diese repetitiven Abbremsmechanismen führen zu deren Überbelastung mit konsekutiven Rupturen. Diese können unterschiedliche Ausmaße annehmen und reichen von inkompletten Längsrissen bis zu komplexen Rupturformen und degenerativen Zerfaserungen. Nicht selten ist die daraus entstehende Symptomatik die Erstmanifestation der chronischen VKB-Instabilität, da viele Patienten die VKBInstabilität subjektiv nicht spüren und sogar sportliche Aktivitäten ausführen können.

\section{Klinische Diagnostik}

\section{Akute VKB-Läsion}

Klinisch stehen Schmerzen und oft pralle Gelenkergüsse im Vordergrund. Bei einer massiven Schwellung ist das Gelenk kaum zu untersuchen. In diesem Fall empfiehlt sich die Punktion des Hämarthros. Eine Akutarthroskopie zur Abklärung der intraartikulären Veränderungen ist nicht zu empfehlen (s. unten).
Der typische Test für eine VKB-Insuffizienz, der Pivot-Shift-Test ist im akuten Stadium wegen der schmerzhaften Abwehrspannung kaum zu prüfen. Demgegenüber ist der Lachman-Test, die extensionsnahe vordere Schublade in $20-30^{\circ}$ Knieflexion sehr einfach zu untersuchen (Abb.8). Der Patient wird dabei aufgefordert zu entspannen, ein ablenkendes Gespräch oder eine Frage z.B. nach den sportlichen Aktivitäten ist hier hilfreich. Lässt sich kein fester Anschlag finden, ist die Diagnose einer VKB-Ruptur gegeben. Manchmal lässt sich der Lachman-Test aber erst nach Entlastung eines „schmerzhaften“ Hämarthros prüfen. Sollten starke Schmerzen die Beurteilung verhindern, wird die Untersuchung nach 2 bis 3 Tagen wiederholt.

\section{Chronische Läsionen}

Es wird sowohl der Lachman-Test als auch der Pivot-Shift-Test geprüft. Hierbei wird das Kniegelenk unter leichten Valgusstress gesetzt und aus gestrecktem Zustand in die Beugung bzw. umgekehrt bewegt. Bei einer VKB-Insuffizienz kommt es extensionsnah zur anterioren Subluxation und zu einer spontanen Reposition ab ca. $40-45^{\circ}$ bedingt durch die Änderung der Zugrichtung des Tractus iliotibialis. Die Auslösung des Pivot-Shift-Tests ist sehr von der Erfahrung des Untersuchers abhängig. Gleichfalls wird der Lachman-Test geprüft (s. oben). Weist dieser keinen festen Anschlag auf, ist die Diagnose einer VKBInsuffizienz gegeben. Gleichfalls werden Meniskusläsionen, eine Lockerung des medialen und lateralen Kapsel-BandApparates, aber auch ein intraartikulärer Erguss ausgeschlossen bzw. bestätigt.

Bei einem positiven Lachman-Test $(3+)$ mit festem Anschlag muss auch an eine HKB-Läsion gedacht werden.

\section{Instrumentelle Stabilitätsprüfung}

Die Quantifizierung des vorderen Schubladenausmaßes ist durch die klinische Untersuchung nur unzureichend möglich. Vielfach wird in die Grade $+(2-5 \mathrm{~mm})$, ++ (6-10 mm) und +++ (mehr als $10 \mathrm{~mm}$ ) unterteilt. Eigene Untersuchungen zeigten, dass gerade in den Grenzbereichen der einzelnen Gradzuordnungen nur zu 50\% richtig quantifiziert wird. Für einen Untersucher ist es extrem schwer zu unterscheiden, ob das vordere Schubladenausmaß 8 oder $11 \mathrm{~mm}$ beträgt. Daher empfiehlt sich 


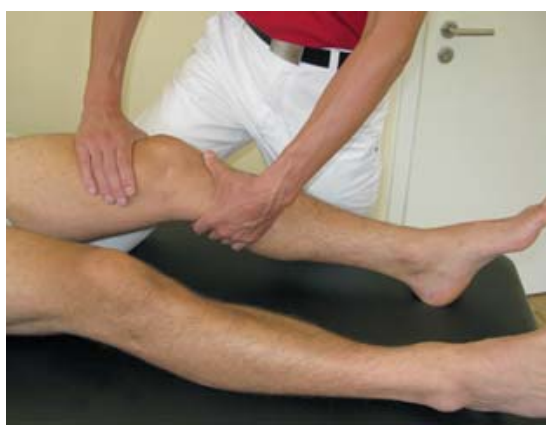

Abb. 8 Lachman-Test. Das Knie des Patienten liegt auf dem Oberschenkel des Untersuchers. Somit ist bei der Prüfung des sog. "stabilen Lachman-Tests" eine Abwehrspannung des Patienten leicht festzustellen.

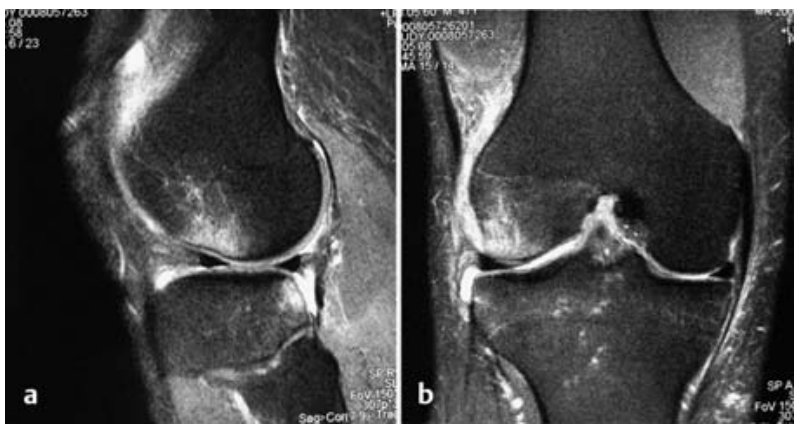

Abb. $10 \mathrm{a}$ und $\mathbf{b}$ Bone Bruise 2 Tage nach VKBRuptur im vorderen Anteil des lateralen Femurkondylus und posterior im lateralen Tibiaplateau (a). Im seitlichen Strahlengang zeigt sich die Größe des Knochenödems im lateralen Femurkondylus (b).

zur Objektivierung der pathologischen Tibiabewegung die instrumentelle Stabilitätsprüfung mit dem KT-1000 [15, 16] (Abb.9). Der Test wird als maximal manueller Test geprüft. Das Ausmaß der Tibiaverschiebung ist auf der Anzeigeskala abzulesen. Da immer beide Kniegelenke untersucht werden, wird durch Bildung der Seit-zu-Seit-Differenz die pathologische Schubladenbewegung bestimmt. Der Referenzwert der intakten Seite gilt als Hinweis auf die physiologische Laxität des Gelenks. Zu bedenken bleibt aber, dass bei Werten von $>10 \mathrm{~mm}$ auf der „gesunden Seite“ der Verdacht einer beidseitigen VKB-Läsion besteht. In diesen Fällen wird auch die gesunde Seite differenziert untersucht (Anamnese, Lachman-Test, Pivot-ShiftTest).

\section{Bildgebende Diagnostik}

Röntgen

Nach jeder frischen Verletzung erfolgen Röntgenaufnahmen in 2 Ebenen zum Ausschluss einer knöchernen Läsion. Knöcherne Bandausrisse, insbesondere bei Jugendlichen und Kindern, sind nicht selten.
Auch bei chronischen VKB-Läsionen ist die Röntgenuntersuchung obligat. Die Aufnahmen können als traditionelle a.-p. und seitliche Röntgenaufnahme angefertigt werden. Bei uns hat es sich jedoch bewährt, die a.-p. Aufnahme als p.-a. Belastungsaufnahme in der sog. „Rosenberg-Technik“ anzufertigen. Hierbei erfolgt die Aufnahme im p.-a. Strahlengang in $45^{\circ}$ Flexion im Stehen [68]. Bei diesem Flexionsgrad werden genau Hauptbelastungszone entsprechen. Damit sind Gelenkspaltverschmälerungen als Hinweis auf Knorpelläsionen spezifischer und sensitiver zu dokumentieren. Ebenfalls erhält man eine Übersicht über die Area intercondylaris.

Die seitliche Röntgenaufnahme wird je nach Fragestellung als $30^{\circ}$ gehaltene Aufnahme unter vorderem Schubladenstress im Scheuba-Apparat (gehaltener Lachman-Test) [58] oder als Hyperextensionsaufnahme angefertigt.

Die maximale Extensionsaufnahme ist bei hyperlaxen Patienten mit einseitiger oder beidseitiger Überstreckung, aber auch bei Streckdefiziten oder nach bereits erfolgter VKB-Rekonstruktion, hilfreich [83]. Es ist nicht nur das Ausmaß die Knorpelareale belastet, die der des Streckdefizits bzw. der Hyperextension zu bestimmen, sondern man findet auch mögliche Ursachen für pathologische Veränderungen wie z.B. Osteophyten in der Area intercondylaris anterior oder fehlplatzierte Bohrkanäle nach Voroperationen.

\section{MR-Tomografie (MRT)}

Die MRT nimmt bei der Kreuzbanddiagnostik einen wichtigen Stellenwert ein. Insbesondere nicht erfahrene Untersucher, aber auch Patienten „fordern“ quasi schon bei jedem Verdacht einer Bandverletzung eine MRT. Man muss sich jedoch immer bewusst sein, dass mit der MRT lediglich eine morphologische Darstellung der Bandstrukturen möglich ist. Eine funktionelle Aussage über das Ausmaß einer Instabilität ist nicht möglich. Bei frischen Läsionen findet sich ein breites Befundspektrum, das von Unterblutungen bis hin zu kompletten und inkompletten Rupturen reicht.

Bei frischen VKB-Rupturen sind häufig Knochenödeme (Bone Bruise) nachzuweisen. Diese finden sich bevorzugt im lateralen Femurkondylus, hier im Bereich der Grenzrinne und im dorsalen Teil des lateralen Tibiaplateaus (Abb.10). Bei ausgedehnten Brisanztraumen können diese Knochenödeme aber auch den kompletten lateralen Femurkondylus und das laterale Tibiaplateau, seltener den medialen Femurkondylus umfassen. Bei Hyperextensionstraumen sind auch Impressionsfrakturen im Bereich der lateralen Grenzrinne (lateral notch fracture) zu finden (Abb.11).

Bei chronischen VKB-Läsionen wird die VKB-Struktur vom Radiologen nicht selten als Partialruptur oder Vernarbung beurteilt. Entscheidend für die weitere Therapie ist aber nicht der MRT-Befund, sondern der Befund der klinischen Untersuchung in Korrelation mit der subjektiven Symptomatik des Patienten.

Ein typischer Befund bei einer chronischen VKB-Insuffizienz ist ein syphonartiger Verlauf des HKB. Dieser Verlauf wird manchmal vom Radiologen als Hinweis auf eine HKB-Läsion gedeutet.

Über den Zustand der Menisken, insbesondere im Bereich der Hinterhörner und des Knorpels, kann die MRT zusätzlich hilfreiche Auskünfte geben. 
Arthroskopie

\section{Akute Läsion}

Die Arthroskopie bei der frischen VKBRuptur und einem Hämarthros ist nur aus Gründen der Diagnostik nicht indiziert.

Noch vor Jahren galt die Devise ,jedes Hämarthros soll bzw. muss arthroskopisch abgeklärt werden“. Von dieser Forderung sollte man sich heute distanzieren. Durch die Arthroskopie bei einem Hämarthros wird das Blut zwar aus dem intraartikulären Gelenkraum herausgespült, nicht selten werden aber, um den intraartikulären Zustand $\mathrm{zu}$ beurteilen, Teile des Hoffa-Fettkörpers reseziert. Dies kann zur Fibrose des Fettkörpers mit Narbenzügen und postoperativen Schmerzen führen. Darüber hinaus kann das Gelenk durch die Arthroskopie auch in eine Kapselfibrose überführt werden. Dieser Zustand ist dann sehr schwierig $\mathrm{zu}$ therapieren. Zudem werden leider häufig rupturierte Kreuzbandstümpfe oder elongierte Kreuzbandfasern bei der „Akutarthroskopie“ reseziert, ohne dass hierfür eine therapeutische Notwendigkeit besteht. Die Kreuzbandstümpfe bieten bei der definitiven VKB-Rekonstruktion (s. unten) eine wichtige Orientierungshilfe.

Es lässt sich feststellen, dass oft keine therapeutischen Konsequenzen durch die Arthroskopie des Hämarthros zu erzielen sind (Abb. 12). Die diagnostischen Möglichkeiten lassen sich wesentlich besser durch die sorgfältige klinische Untersuchung und mit der MRT erfassen, unter Ausschluss der oben angegebenen Risiken. Ein schmerzhaftes Hämarthros wird punktiert und Lymphdrainagen rezeptiert. Ein oder 2 Tage danach erfolgt eine erneute klinische Untersuchung, sollte diese nach der Punktion nicht möglich sein.

Findet sich jedoch eine ausgedehnte Bewegungseinschränkung, z.B. eine akut aufgetretene Streckhemmung, ist die Arthroskopie gerechtfertigt. Hierfür sind meist Meniskusläsionen (Korbhenkelläsionen) verantwortlich. In diesem Fall liegt meist eine alte VKB-Läsion vor, da die Kombination von frischer VKB-Läsion und einer Korbhenkelläsion eher selten anzutreffen ist.

Früher wurde häufig von der sog. „unhappy triad“ ausgegangen, bei der das VKB, das mediale Seitenband und der Innenmeniskus verletzt waren. Die heu- tigen klinischen Erfahrungen zeigen aber, dass diese Verletzungskombination eher selten ist. Wesentlich häufiger als der Innenmeniskus ist bei der akuten VKB-Läsion das Außenmeniskushinterhorn verletzt. Dies korreliert auch mit MRT-Befunden (Bone Bruises im dorsalen Bereich des lateralen Tibiaplateaus). Bei der Arthroskopie finden sich häufig Zerstörungen der gesamten posterioren Meniskusstruktur. Bei einer Akutarthroskopie wird hier oft eine Resektion angestrebt, da man nicht glaubt, dass es zu einer Heilung kommen kann. Die klinischen Erfahrungen belegen aber anderes. Bei einer abwartenden Haltung findet sich nach 6 bis 8 Wochen zum Zeitpunkt der definitiven VKB-Versorgung oft eine Reparatur mit nur noch minimalen Zungenläsionen. Daher sollte bei einer vermeintlichen Akutarthroskopie in diesem Bereich äußerst vorsichtig vorgegangen und auf eine Meniskusresektion verzichtet werden.

\section{Chronische Läsion}

Der arthroskopische Befund reicht von einer kompletten Ruptur bis hin zum scheinbar intakten VKB. Bei Rupturen finden sich kolbenförmige Verdickungen der rupturierten VKB-Bündel, die zu einem Streckdefizit führen können (Abb.13). Das VKB kann aber auch komplett fehlen.

Nicht selten ist das rupturierte VKB auf das HKB aufgewachsen, sodass die Innenseite des lateralen Femurkondylus nach dorsal frei passierbar ist. Entscheidende Bedeutung hat die Palpation mit dem Tasthaken. Spannt sich die VKBStruktur nicht an, darf aber nicht automatisch von einer VKB-Läsion ausgegangen werden (Abb.14). Theoretisch könnte auch eine HKB-Läsion vorliegen. Hierbei gerät der Tibiakopf in eine posteriore Position. In dieser zurückgesunkenen Position ist das VKB zwangsweise gelockert bzw. erscheint elongiert. Die Diagnose der „VKB-Elongation“ darf aber nicht automatisch das weitere therapeutische Handeln auf das VKB richten. Vielmehr muss unbedingt zunächst eine HKB-Läsion ausgeschlossen werden $[84,88]$ (s. unten).

Knorpelläsionen finden sich sehr häufig. Diese korrelieren mit dem Alter und dem Ausmaß der Instabilität. Von Knorpelfissuren bis hin zu freiliegenden Knochenarealen reicht das Spektrum der Knorpelveränderungen (Abb.15). An den Menisken sind sowohl inkomplette als auch komplette Längsrisse, insbe- sondere in den Hinterhornbereichen zu finden. Besonderes Augenmerk verdient die sog. „Rampenläsion“. Hierbei handelt es sich um eine Läsion der Aufhängung des Innenmeniskushinterhorns. Bei derartigen Läsionen ist die Resektion gleichbedeutend einer kompletten Meniskektomie. Daher sollte bei derartigen Läsionen eine Refixation angestrebt werden (Abb. 6).

Auch die peripheren Bandstrukturen lockern bei chronischen Instabilitäten konsekutiv aus, sodass nicht selten Insuffizienzen des medialen und posteromedialen und/oder der lateralen und posterolateralen Bandstrukturen vorliegen.

\section{Therapeutisches Vorgehen}

In zahlreichen Studien wurde belegt, dass es nach einer VKB-Ruptur zu tief greifenden Änderungen der Gelenkmechanik kommt $[6,10,42,45,73]$. Zudem steigt durch die Instabilität die Knorpelbelastung, sodass degenerative Knorpelschäden ebenso wie Meniskusläsionen entstehen. Demgegenüber sind auch Untersuchungen bekannt, in denen auch nach einer VKB-Rekonstruktion arthrotische Veränderungen beobachtet wurden [45]. So stellt sich die Frage: „Kann eine VKB-Rekonstruktion die arthrotischen Veränderungen im Gelenk verhindern?" Das Argument oder der Wunsch, dass mit einer VKB-Rekonstruktion Knorpelschäden oder die Arthrose $z u$ verhindern sind, konnte bisher durch keine wissenschaftliche Studie belegt werden. Andererseits muss man feststellen, dass eine Studie, die diese Frage beantworten soll, äußerst problematisch ist. Ein Patient mit einem instabilen Kniegelenk belastet sein Gelenk völlig anders als einer mit einem stabilisierten Kniegelenk. Daher sind zwar die Behandlungsparameter vergleichbar, das patientenspezifische Aktivitätsniveau unterscheidet sich aber erheblich. So können Patienten nach erfolgreicher VKB-Rekonstruktion oft ihre sportliche Aktivität in fast gleichem Niveau wie vor der Ruptur fortführen. Patienten mit einer VKB-Instabilität erreichen dieses Niveau nur extrem selten.

Bei der Therapieentscheidung gilt es den Aktivitätsgrad, d.h. die Art der sportlichen Aktivität, das Alter, die Kooperationsfähigkeit sowie auch die subjektiven Ziele und Erwartungen des Patienten auszuloten. 


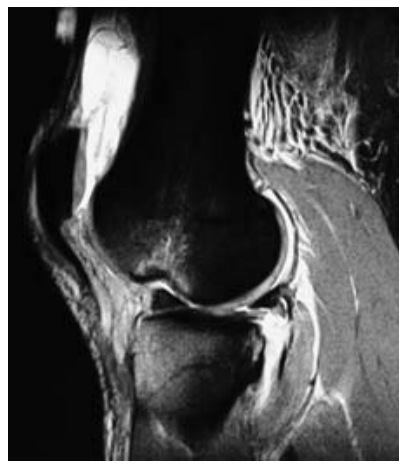

Abb. 11 Laterale Notchfraktur.

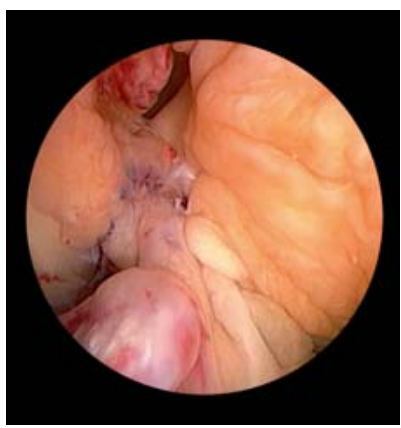

Abb. 13 VKB-Ruptur (4 Wochen alt).

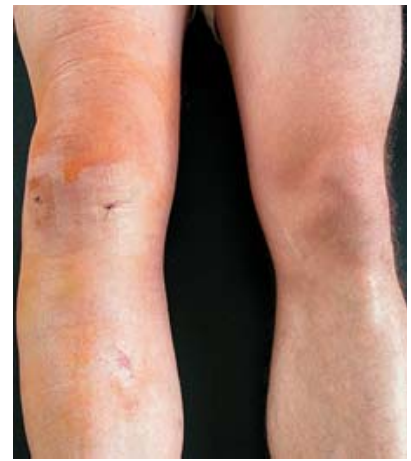

Abb. 12 24-jähriger Patient 6 Tage nach VKB-Ruptur. Am Skiort wurde eine Arthroskopie zur Sicherung der Diagnose durchgeführt und eine VKB-Läsion diagnostiziert sowie abgerissene Kreuzbandanteile entfernt. Gleichzeitig wurde eine ausgedehnte Ruptur der gesamten lateralen Kapsel „diagnostisch gesichert“. Wegen dieser Läsion wurde der Patient zugewiesen. Es findet sich ein deutlich geschwollener Unterschenkel (V.a. Thrombose) und nicht verheilte Zugänge. Insbesondere am lateralen Arthroskopzugang findet sich eine Sezernierung. Daher ist die notwendige primäre Versorgung der komplex zerrissenen lateralen Strukturen extrem risikoreich.

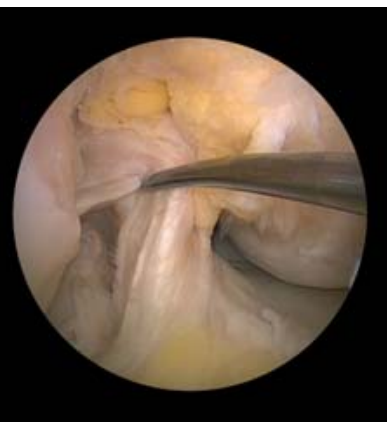

Abb. 14 VKB-Elongation. Die Palpation zeigt ein deutlich elongiertes VKB. Es darf jedoch nicht die Diagnose einer „VKB-Elongation" gestellt werden, bis eine HKB-Läsion ausgeschlossen ist.
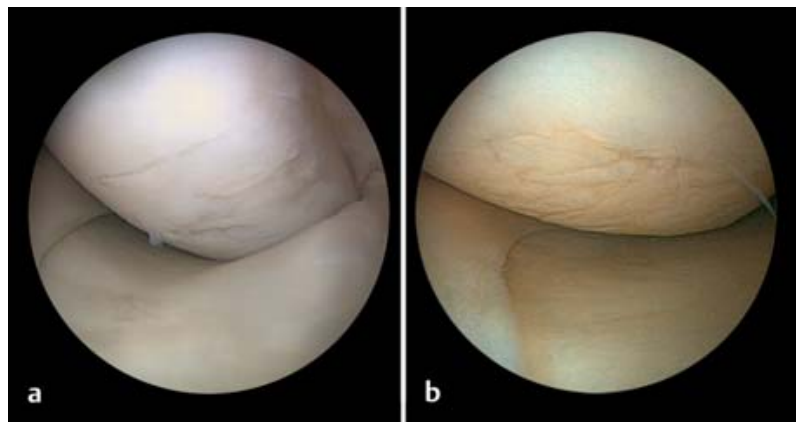

Abb. 15a und $\mathbf{b}$ Knorpelläsionen am medialen Femurkondylus bei chronischer VKB-Insuffizienz. Typische quer verlaufende Knorpelfissuren (VKB-Ruptur vor 8 Monaten) (a). Multiple Knorpelaufbrüche nach VKB-Ruptur vor 3,5 Jahren (b).
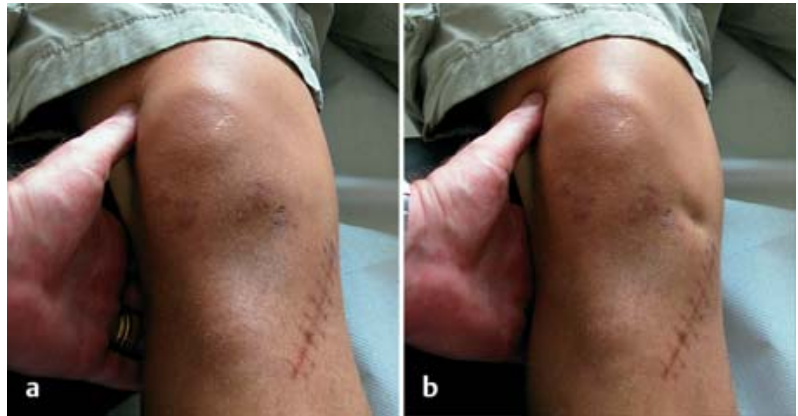

Abb. 16a und b Prüfung der medialen Instabilität. Bei dem 32-jährigen Patienten wurde vor Jahren eine Innenbandruptur operativ versorgt. Vor 6 Monaten zog sich der Patient eine VKBRuptur zu. Die Inspektion zeigt die reizlose Narbe nach operativer Versorgung des medialen Seitenbands (a). Die Prüfung der medialen Aufklappbarkeit führt zu einem Vakuumphänomen als Ausdruck der medialen Bandinsuffizienz (b). Zur VKB-Rekonstruktion ist die Verwendung der ipsilateralen Hamstringsehne nicht indiziert. Vielmehr muss evaluiert werden, ob der mediale Bandapparat zu stabilisieren ist.

Bei einer Sportart besteht insbesondere dann ein hohes Risiko des Pivotierens, wenn diese Elemente von Richtungsänderungen und Rotationsbewegungen beinhaltet. Diese Bewegungen treten eher selten z.B. beim Laufen und Skifahren auf. Betreiben die Sportler diese Sportarten jedoch auf höherem oder höchstem Leistungsniveau, ist auch hier eine operative Versorgung zu empfehlen.

\section{Akute Läsion}

Bei der akuten VKB-Ruptur gilt es zunächst die akute Symptomatik zu therapieren. Ein praller Gelenkerguss wird punktiert, um die Schmerzsymptomatik zu reduzieren. Mit dem Patienten muss dann ein Therapiekonzept erarbeitet werden, das folgende Faktoren berücksichtigt:

1. Sportart. Betreibt der Sportler eine Sportart, die mit Verdrehmechanismen oder schnellem Richtungswechsel verbunden ist, bietet sich eine konservative Versorgung nicht an. Hier sollte eine operative Versorgung angestrebt werden. Der Zeitpunkt der operativen Versorgung hängt im Wesentlichen vom Zustand des Kniegelenks ab (s. unten). Bei Sportarten wie Radfahren und Schwimmen treten plötzliche Richtungsänderungen dagegen kaum auf, sodass hier eine operative Versorgung der VKB-Ruptur nicht akut indiziert ist. Sollten sich im chronischen Stadium Beschwerden einstellen, wird die VKB-Rekonstruktion empfohlen. 
2. Leistungsniveau. Ist der Patient Berufs- oder Hochleistungssportler, ist eine operative Versorgung möglichst schnell anzustreben, um nicht unnötig Zeit zu verlieren. Dennoch gilt es zu berücksichtigen, dass der Sportler nicht primär möglichst schnell wieder sportlich fit sein soll, sondern seine sportliche Aktivität möglichst lange (wenn möglich über Jahre) erhalten soll. Dies ist dem Sportler, seinem Manager bzw. Betreuer nicht immer einfach zu vermitteln.

3. Begleitverletzungen. Begleitverletzungen, die mit der VKB-Läsion aufgetreten bzw. entstanden sind, gilt es ebenfalls zu beachten. Insbesondere gilt es, periphere Instabilitäten zu erfassen (Abb. 16). Findet sich eine deutliche Aufklappung (mediale Aufklappung und/oder laterale Aufklappung) in Streckstellung oder sogar Überstreckung, muss mit einer schwerwiegenden Kapsel-Band-Läsion unter Beteiligung der dorsomedialen bzw. dorsolateralen Kapsel gerechnet werden. In einem derartigen Fall sollte eine primäre Versorgung der rupturierten Strukturen erwogen werden.

4. Zustand des Kniegelenks. Lange Zeit galt es, eine akute Verletzung innerhalb der ersten Stunden oder Tage nach der Verletzung zu versorgen. Dies führte dazu, dass insbesondere bei Verwendung des mittleren Drittels der Patellarsehne (BTB-Technik) erhebliche Bewegungseinschränkungen, manchmal sogar fibroarthrotische Reaktionen im postoperativen Verlauf aufgetreten sind $[51,67,74]$. Daher wurde in den vergangenen Jahren zu einem verzögert primären Vorgehen geraten. Das Kniegelenk sollte sich von der akuten Verletzung „erholen“ und wieder frei beweglich und reizlos sein [74]. Findet sich eine Kapselschwellung und ist das Gelenk schmerzhaft und kaum bewegbar, sollte auf eine primäre Versorgung verzichtet werden. Insbesondere wenn Partialrupturen des Seitenbands und der dorsomedialen Kapsel vorliegen, gestaltet sich zunächst das konservative Vorgehen wesentlich günstiger. Ist ein reizfreier Zustand, z. B. nach 4 bis 7 Wochen erreicht, kann die definitive Versorgung durchgeführt werden.

Findet sich dagegen nach der VKBRuptur ein reizloses, schmerzfreies Gelenk ohne Kapselschwellung mit ausreichender Beugung (Flexion $>110^{\circ}$ ), kann direkt die definitive Versorgung erfolgen [83]. Der Patient ist aber darüber aufzuklären, dass, falls sich postoperativ Probleme einstellen, der Operateur zu kontaktieren ist. Daher ist der enge Kontakt zwischen nachbehandelnden Physiotherapeuten bzw. Vereinsarzt und Operateur anzustreben. Ergeben sich bei der Nachbehandlung Bewegungseinschränkungen oder Reizzustände, muss das weitere therapeutische Vorgehen gemeinsam diskutiert werden.

Je höher der Leistungsstand des Patienten ist, umso eher wird eine akute Versorgung angestrebt. Dennoch sollte man sich nicht dazu verleiten lassen, sehr gereizte, schmerzhafte und bewegungseingeschränkte Kniegelenke zu stabilisieren. Bestehen Zweifel, ob man den Sportler akut operieren oder warten sollte, ist im Zweifelsfall das verzögerte Vorgehen zu empfehlen. Die 3 oder 4 Wochen Zeitverlust lassen sich leicht bei der Nachbehandlung wieder aufholen.

Zeigt sich im MRT ein erhebliches Bone Bruise, sollte die Versorgung nach 4 bis 6 Wochen angestrebt werden. Das Kniegelenk hat dann meist wieder einen reizlosen oder reizärmeren Zustand erreicht.

Bei Patienten mit geringem Sportniveau und bei Freizeitsportlern sollte, wenn ein Alter von 40 Jahren überschritten ist, eher die verzögerte Versorgung angestrebt werden. Aus der eigenen klinischen Erfahrung ist bekannt, dass gerade bei Frauen, die älter als 40 Jahre sind, erhebliche postoperative Bewegungsstörungen auftreten können, wenn die Versorgung innerhalb der ersten Tage erfolgt. Dies ist besonders dann der Fall, wenn das mediale Seitenband partiell oder komplett rupturiert ist. Nicht selten findet man danach eine verzögerte Mobilisierbarkeit, einen relativ lange persistierenden Reizzustand mit Schwellungen, manchmal sogar algodystrophe Reaktionen. Dieses Risiko lässt sich reduzieren, wenn verzögert primär vorgegangen wird. Erst wenn das Gelenk völlig reizlos und wieder nahezu uneingeschränkt beweglich ist, sollte die VKBRekonstruktion erfolgen. Im Zweifelsfall sollte bei Nichterreichen der Beweglichkeit durch die Physiotherapie eine arthroskopische Arthrolyse erfolgen.

Viele Sportler verletzen sich nicht beim Wettkampf, sondern im Urlaub. Der Skisport ist hier besonders verletzungsträchtig. Der akut verletzte Sportler be- gibt sich hier oftmals zum Ausschluss von knöchernen Verletzungen zum nächstgelegenen Krankenhaus oder zum „Spezialisten am Skiort“. Gerade bei privatversicherten Verletzten hat sich in zahlreichen Skiorten eine Akuttherapie entwickelt, die seriösen medizinischen Erkenntnissen oft entgegensteht. So werden häufig diagnostische Arthroskopien mit Resektion rupturierter Bandanteile durchgeführt. Von einigen Kollegen in Skiorten werden sogar Akutarthroskopien an beiden verletzten Kniegelenken in einer Sitzung vorgenommen, ohne jegliche therapeutische Konsequenz. Zudem werden oft akute Kreuzbandverletzungen, geradezu notfallmäßig, versorgt, unabhängig davon, ob Begleitverletzungen vorliegen und unabhängig vom Zustand des Gelenks. Dieses überhastete Vorgehen führt oft zu schwerwiegenden postoperativen Bewegungseinschränkungen und massiven Gelenkfibrosen, die teilweise eine monatelange Nachbehandlung nach sich ziehen. Daher sollte der Sportler, wenn er sich im Skiurlaub verletzt, nicht nur Kontakt mit dem ortsansässigen Behandler aufnehmen, sondern wenn möglich einen Orthopäden „in der Heimat" kontaktieren, um sich das vorgeschlagene therapeutische Konzept bestätigen zu lassen. Oft ist eine abwartende Haltung für das Kniegelenk besser als die forcierte Indikationsstellung am Skiort.

\section{Chronische Läsion}

Bei chronischen VKB-Insuffizienzen wird in der Literatur auch über konservative Therapiekonzepte berichtet [6, $10,33,34]$. Sportler mit geringem oder mittleren Leistungsniveau können recht gut mit der chronischen Instabilität zurechtkommen. Ist bei der sportlichen Aktivität normalerweise eine gute muskuläre Anspannung und Kontrolle vorhanden, wird keine Instabilität verspürt. Beim Gehen auf unebenen Wegen wird dagegen nicht auf das Kniegelenk geachtet. Daher wird hier manchmal über eine Instabilität und ein Wegschnappen (Pivotieren) berichtet. Gerade mit dieser Patientengruppe muss differenziert diskutiert werden, ob eine operative Stabilisierung erfolgen soll oder nicht. Auch bei einer nicht symptomatischen Instabilität treten Meniskus- und Knorpelschädigungen auf. Diese können nach einer Latenzzeit in den Vordergrund der klinischen Symptomatik treten. Wie schnell Meniskus- und Knorpelläsionen auftreten, lässt sich aber nicht vorher- 
sagen. Die gefährliche Problematik der chronischen VKB-Insuffizienz besteht darin, dass oft ein langes symptomfreies Intervall vorliegt. Deshalb sehen diese Patienten auch nicht unbedingt den Sinn der operativen Stabilisierung.

Beim aktiven und vor allem erfolgreichen Sportler mit einer VKB-Insuffizienz ist der Zeitpunkt des operativen Vorgehens schwer festzulegen. Saisonverlauf und das persönliche Sportziel sind wichtige Begleitparameter, die im therapeutischen Management berücksichtigt werden müssen.

Radiologisch sichtbare degenerative Veränderungen wie z.B. Osteophyten oder eine Gelenkspaltverschmälerung in den Belastungsaufnahmen (Rosenberg-Aufnahme, s. oben) sollten nicht Anlass sein, die operative Therapie abzulehnen [73]. Vielmehr muss mit dem Sportler besprochen werden, dass bei der bestehenden Instabilität arthrotische Veränderungen insbesondere Meniskusschäden kaum zu vermeiden sind, besonders wenn das Gelenk weiter sportlich belastet wird. Ist der Sportler (z.B. Hobbysportler) jedoch bereit, seine sportliche Aktivität zu reduzieren und bemerkt er im Alltag und auch bei sportlichen Aktivitäten keine Instabilität, kann durchaus ein operatives Vorgehen gegen ein konservatives Vorgehen abgewogen werden.

Die konservative Therapie bei chronischer VKB-Insuffizienz muss kritisch betrachtet werden. Kann sich jemand aus beruflichen oder sportlichen Gründen nicht zu einem operativen Vorgehen entscheiden, sollte dies Anlass sein, den Patienten engmaschig zu kontrollieren. Bei Auftreten von klinischen Symptomen, wie rezidivierenden Schwellungen, Meniskusläsionen mit Einklemmungen und Knorpelschäden, sollte unbedingt ein operatives Vorgehen favorisiert werden. Der Sportler sollte darüber informiert werden, dass, wenn das Gelenk wiederholt anschwillt, meist Knorpelschäden und Meniskusschäden vorliegen und dies oft der „Anfang vom Ende“ des Gelenks ist.

Das Ziel jeder VKB-Rekonstruktion besteht darin, den natürlichen Verlauf einer VKB-Insuffizienz mit funktioneller Instabilität und sekundärer Schädigung von Meniskus- und Knorpelstrukturen aufzuhalten oder zumindest signifikant zu verzögern. Trotz zahlreicher Studien ist es nach wie vor nicht eindeutig ge- klärt, welche Patienten instabilitätsbedingte Schäden an Menisken und Knorpel entwickeln und welche Patienten nicht. Eine funktionelle Instabilität bei VKB-Insuffizienz findet sich bei 60$100 \%$ der Patienten.

Folgenden Patienten sollte unbedingt eine VKB-Rekonstruktion empfohlen werden:

1. Junge und sportlich aktive Patien-

ten. Insbesondere der junge aktive Sportler strebt häufig das gleiche Aktivitätsniveau wie vor der Verletzung an.

2. Sportler mit funktioneller Instabilität im täglichen Leben oder bei der sportlichen Aktivität. Bei Instabilität im Alltag oder bei sportlicher Aktivität wird die VKB-Stabilisierung empfohlen.

3. Chronische VKB-Insuffizienz und neu aufgetretenen Meniskusläsionen. Intraartikulär finden sich meist erhebliche Veränderungen. Die Meniskussymptomatik ist häufig das erste richtige klinische Zeichen, das zum Arztbesuch führt. Bei dieser Patientengruppe sollte die Indikation zur Meniskusrefixation großzügig gestellt werden, da ein langfristig gutes Ergebnis nach VKB-Rekonstruktion insbesondere dann zu erwarten ist, wenn ein intakter oder refixierter Meniskus vorliegt. Zudem sind die Heilungschancen der Meniskusrefixation wesentlich größer, wenn in gleicher Sitzung eine VKB-Rekonstruktion durchgeführt wird, da hierbei zahlreiche Stammzellen freigesetzt werden.

4. Komplexinstabilitäten. Bei VKB-Instabilität und Insuffizienz einer peripheren Struktur, z.B. Läsion des medialen bzw. lateralen Seitenbands und/oder der posterolateralen Gelenkecke, ist die VKB-Rekonstruktion indiziert, da die VKB-Insuffizienz hierbei wesentlich symptomatischer ist. In gleicher Sitzung sollte aber auch die Versorgung der insuffizienten medialen und/oder lateralen Gelenkecke angestrebt werden. Wird bei einer signifikant peripheren Instabilität nur das VKB rekonstruiert, muss mit einer Auslockerung des Transplantats gerechnet werden.

5. Kinder und Jugendliche. Bei Kindern und Jugendlichen treten gehäuft Knorpel- und Meniskusläsionen auf [4]. In dieser Patientengruppe ist es zudem sehr schwierig, die sportliche und freizeitliche Aktivität so zu limitieren, dass das Gelenk nicht belastet bzw. geschont wird. Eine konservative
Behandlung zur Vermeidung von Subluxationsphänomenen lässt sich nicht erfolgreich durchführen (Abb.17).

Die Befürchtung, dass die VKB-Rekonstruktion gravierende Wachstumsstörungen hinterlässt, hält auch heute noch zahlreiche Kollegen und insbesondere auch Eltern davon ab, Kinder operieren zu lassen. Zahlreiche Untersuchungen haben aber gezeigt, dass ein Durchbohren der Wachstumsfugen nur extrem selten $\mathrm{zu}$ einer Wachstumsstörung führt. Daher sollte die Indikation zur VKB-Rekonstruktion in dieser Patientengruppe eher sehr großzügig gestellt werden $[4,17$, 56]. Es gilt jedoch, die adäquate Operationstechnik zu wählen. Durch Vorabinformationen über verschiedene Medien oder durch nicht ausreichend informierte Kollegen anderer Fachgebiete sind die Eltern häufig verunsichert und ängstlich, ob ihre Kinder auch von der Stabilisierung profitieren, auch in Anbetracht des Risikos eines Fehlwachstums. Diese Unsicherheit lässt sich relativ einfach klären. Man sollte den Eltern 2 Möglichkeiten darlegen. Im Fall einer VKB-Rekonstruktion kam es früher sehr selten zu Wachstumsstörungen. Diese sind aber, entsteht z.B. ein Genu varum oder valgum, später durchaus korrigierbar. Treten bei den jungen Patienten jedoch gravierende Meniskus- und Knorpelläsionen auf, sind die therapeutischen Möglichkeiten äußerst beschränkt. Bei Knorpelläsionen besteht zwar die Möglichkeit der Knorpeltransplantation, sollte jedoch eine Meniskusresektion notwendig sein, ist die Prognose wesentlich ungünstiger. Auch heute ist noch kein adäquates Meniskustransplantat oder ein adäquater Meniskusersatz erhältlich. Erste Erfahrungen und auch Erfolge sind zwar mit einem Kollagenersatz (CMI-Implantat) vorhanden, Erfahrungen an größeren Patientenkollektiven stehen aber noch aus. Stellt man den Eltern diese beiden Möglichkeiten gegenüber, ist die Entscheidung meist relativ einfach.

Ist man sich unsicher, ob der Patient von einer VKB-Rekonstruktion profitiert, sollte man ihn nicht zur Operation überreden. Viel sinnvoller ist es, dem Patienten eine VKB-Orthese (z.B. DonJoy Fource-Point ACL, Fa. Ormed.djo, Freiburg) zu verordnen (Abb. 18). Führt das Tragen dieser Schiene zur Reduktion der klinischen Symptomatik (keine Instabilität, Ergussneigung rückläufig, Schmer- 


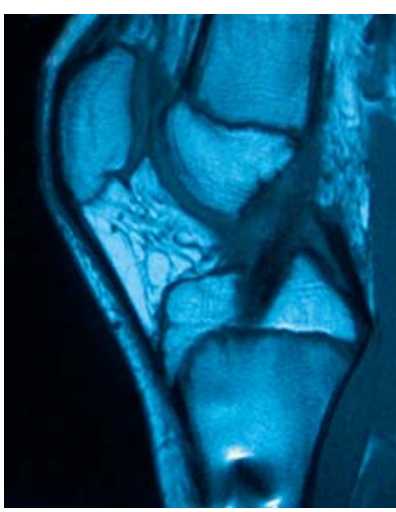

Abb. 17 VKB-Rekonstruktion bei 7-jähriger Patientin. MR postoperativ. Die Patientin ist 1 Jahr postoperativ wieder uneingeschränkt sportfähig.

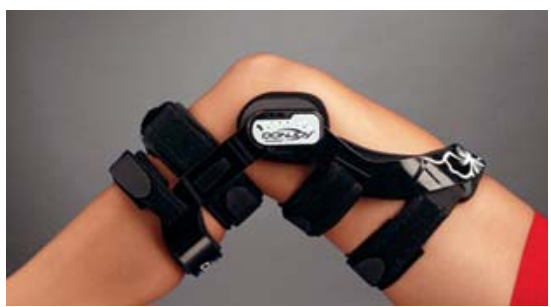

Abb. 18 Donjoy FourcePoint-ACL-Schiene mit speziellem Scharniergelenk und integriertem Abbremssystem (Fa. Ormed.djo, Freiburg).

zen rückläufig), ist eine VKB-Rekonstruktion zu empfehlen. Bleibt eine Verbesserung der klinischen Symptomatik völlig aus, ist die Stabilisierung eher kritisch abzuwägen.

Eine weitere Entscheidungshilfe bei der Indikationsstellung kann eine Arthroskopie sein. Zeigen sich instabilitätsbedingte Knorpel- und Meniskusläsionen, deutet dies darauf hin, dass die vorliegende Instabilität nicht kompensiert werden kann (Abb.15). In diesem Fall wird eine VKB-Rekonstruktion empfohlen.

\section{Konservative Therapie}

Eine konservative Therapie beim sportlich aktiven Patienten ist nur dann gerechtfertigt, wenn keine klinische Symptomatik vorliegt. Meniskuszeichen, arthrotische Veränderungen oder eine Ergussneigung bzw. eine Instabilität sollten nicht bestehen. Trotzdem werden derartige Patienten regelmäßig ca. einmal pro Jahr - kontrolliert, um eine symptomatische Manifestation der VKB-Insuffizienz rechtzeitig zu erken- nen und gegebenenfalls therapeutisch einzugreifen.

Die vielfach geäußerte Meinung es sei ausreichend die Muskulatur zu kräftigen, um eine VKB-Ruptur zu stabilisieren, ist trügerisch. Da die ischiokrurale Muskulatur agonistisch, der M. quadriceps jedoch antagonistisch zum VKB wirkt, führt ein intensives Auftrainieren des M. quadriceps zu pathologischen anterioren Tibiabewegungen. Bei der klinischen Diagnostik ist dies sogar als ein „aktiver Quadrizeps-Test“ bekannt. Hierbei wird der anteriore Tibiavorschub unter gleichzeitiger QuadrizepsAnspannung bei fixiertem Fuß beobachtet $[16,82]$.

Unter einer physiotherapeutischen Therapie kann es zwar zu einer subjektiven Beschwerdereduktion kommen, die grundlegenden pathomechanischen Mechanismen werden aber dadurch nicht positiv beeinflusst.

\section{Operatives Vorgehen}

Das therapeutische Vorgehen bei VKBRupturen hat sich in den letzten 30 Jahren mehrmals verändert. In den 60erJahren des letzten Jahrhunderts wurde die frische VKB-Ruptur kaum diagnostiziert und dementsprechend therapeutisch vernachlässigt. Bei chronischen Instabilitäten wurden aufwendige extraartikuläre Stabilisierungstechniken beschrieben, die eigentliche Ursache die VKB-Insuffizienz wurde aber nicht angegangen. In den 70er- und 80er-Jahren wurde die Therapie auf die rupturierte VKB-Struktur fokussiert. Zunächst wurde eine frische Naht der Bandstruktur propagiert, was aber nur bei ca. $50 \%$ der Patienten zu einer ausreichenden Stabilität führte. Diese Operation wurde häufig sogar notfallmäßig durchgeführt, um einer Retraktion der Bandstümpfe vorzubeugen. $\mathrm{Zu}$ Beginn der 90er-Jahre fand die Naht bei der akuten Verletzung mit gleichzeitiger Augmentation durch eine körpereigene Sehne oder ein synthetisches Bandmaterial zunehmende Verbreitung. Bedingt durch unbefriedigende Ergebnisse der primären Naht wurde das Augenmerk zunehmend auf rekonstruktive Techniken gesetzt. Sowohl akute als auch chronische VKBLäsionen wurden mit einer Rekonstruktion mit dem mittleren Drittel der Patellarsehne (BTB-Technik) versorgt. Parallel mit der Versorgung der frischen Ruptur durch eine Naht oder Augmentation wurden aber auch Kunstbänder als VKB-Ersatz implantiert $[40,66]$. Das Argument hierfür war die fehlende Entnahmemorbidität bei gleichzeitig sehr schneller Rehabilitation. Daher wählten gerade Sportler zu dieser Zeit gerne diese Therapieoption, um möglichst schnell wieder ihre sportliche Aktivität aufnehmen zu können.

Heute gilt die arthroskopische VKB-Rekonstruktion mit autologen Sehnen als Standardtechnik und wird in verschiedenen Techniken durchgeführt, auf die im Weiteren eingegangen wird.

\section{Spezielle Operationstechnik}

Es gibt wohl kein Band am menschlichen Körper, über dessen Rekonstruktion und operative Versorgung so viel geschrieben worden ist wie zum VKB des Kniegelenks.

\section{Transplantatauswahl}

Zur VKB-Rekonstruktion werden heute ausschließlich körpereigene Transplantate verwendet. Die häufigsten Transplantate sind das mittlere Drittel der Patellarsehne (BTB-Technik) und die Verwendung der Semitendinosussehne (ST-Sehne) gegebenenfalls in Kombination mit der Grazilissehne (GT-Sehne) $[8,14,20,43,49,83,94,101]$.

Diese Techniken unterscheiden sich hinsichtlich der Entnahmetechnik und der damit verbundenen Entnahmemorbidität [42]. Zudem gilt es, in Abhängigkeit des Transplantats die optimalen Verankerungstechniken zu wählen. Bei der BTB-Technik sind Knochenblöcke in den Bohrkanälen zu fixieren. Bei Verwendung der Hamstringsehnen (Semitendinosussehne [ST-Sehne] und Grazilissehne [GT-Sehne]) ist eine effiziente Verbindung zwischen Knochen- und Weichteiltransplantat erforderlich, um eine ossäre Transplantatintegration $\mathrm{zu}$ erreichen.

Im Folgenden wird auf die seltenere Verwendung der Quadrizepssehne oder sonstige autologe Transplantate nicht eingegangen.

\section{Patellarsehne}

Das mittlere Patellarsehnendrittel weist eine Reißfestigkeit zwischen 1700 und $2900 \mathrm{~N}$ auf [57]. Die mechanisch schwächste Stelle des Konstrukts ist der tibial entnommene Knochenblock. Dieser kann bei Belastung frakturieren. Wird diese Insertionsstelle wie beim 
Tab. 2 Mögliche Transplantatentnahmeprobleme nach Entnahme des mittleren Patellarsehnendrittels (BTB-Technik).

1. Patellarfraktur

2. Patellarsehnenruptur

3. Schmerzen beim Hinknien (bis zu $60 \%$ der Patienten) (Abb. 19).

4. Patellarspitzensyndrom

5. Tendinitiden der Patellarsehne

6. femoropatellares Krepitieren

7. infrapatellares Kontraktursyndrom

8. Hoffa-Fibrosen

9. Hernien des Hoffa-Fettkörpers

10. Patella infera

Kreuzbandersatz nicht in axialer Zugrichtung belastet, können Scherkräfte zum Versagen der Bandinsertion am Knochenblock führen. Daher ist aus mechanischer Sicht immer das Gesamtkonstrukt und nicht nur der sehnige Teil der Patellarsehne zu analysieren [94].

Die Entnahme des mittleren Drittels der Patellarsehne stellt einen erheblichen Eingriff in die Integrität des Femoropatellargelenks dar. Es sind zahlreiche Probleme und Komplikationen, die als Donor-Side-Morbidity bezeichnet werden, bekannt [42,43] (Tab. 2).

Das eigentliche Problem besteht aber darin, dass derartige Probleme nur sehr schwer zu therapieren sind (Abb.19). Die Inzidenz und Intensität der Probleme variieren je nach Untersuchung zwischen 4 und $40 \%[2,42,43]$. Daher sollte insbesondere bei Sportlern, die das Femoropatellargelenk besonders belasten, die BTB-Technik zurückhaltend eingesetzt werden. Besteht schon eine femoropatellare Symptomatik, sollte auf die Verwendung dieses Transplantats verzichtet werden.

Hamstringsehnen (ST-Sehne, GT-Sehne) Die Reißfestigkeit der Hamstringsehnen (ST-Sehne, GT-Sehne) wird vom Gesamtquerschnitt des Sehnenkonstrukts bestimmt. Ein einfacher Strang der STSehne weist eine Reißfestigkeit von etwa $1200 \mathrm{~N}$ auf. Bei einem Doppelstrang steigert sich dieser Wert auf $2400 \mathrm{~N}$ und mit einem Vierfachstrang können bis über $4000 \mathrm{~N}$ erreicht werden [22].

Die Entnahmemorbidität ist geringer und meist nur von kurzer Dauer. Manchmal tritt ein Hämatom im Bereich der tibialen Entnahmestelle auf, dass aber meist innerhalb der ersten 2 Wochen

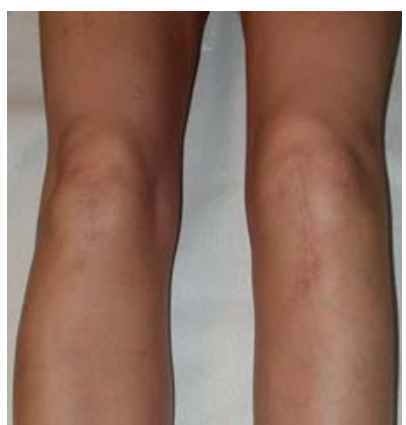

Abb. 19 Donor-Side-Morbidity nach BTB-Technik. 26-jährige Patientin mit beidseitiger VKB-Rekonstruktion in BTB-Technik. Die Patientin klagt beidseits über massive femoropatellare Schmerzen und ist nicht mehr in der Lage sich hinzuknien. Sämtliche konservativen Therapieversuche sind leider fehlgeschlagen.

nach der Operation abklingt. Bei der Nachbehandlung muss auf eine vorsichtige Aufdehnung der Ischiokruralmuskulatur geachtet werden. Bei forcierten Dehnübungen können die Restfasern im Muskelbauch einreißen und zu Einblutungen auf der Oberschenkelrückseite und so zu Schmerzen führen.

Von zahlreichen Operateuren wird standardmäßig die gleichzeitige Entnahme von ST- und GT-Sehne favorisiert, um ein ausreichend langes Transplantat für die Fixation mit einer bestimmten Fixationstechnik zu erhalten [70,92]. Generell ist aber zu hinterfragen, ob die routinemäßige Entnahme der GT-Sehne wirklich notwendig und sinnvoll ist. Die Sehne ist sicherlich nicht deswegen angelegt, um die Anforderungen bestimmter Fixationstechniken $\mathrm{zu}$ befriedigen. Daher wird von uns immer versucht, nur die ST-Sehne als Transplantat zu verwenden. Weist die ST-Sehne eine Länge von 24-26 mm auf, kann ein 4-StrangTransplantat mit einer Länge von 6$6,5 \mathrm{~cm}$ präpariert werden. Lediglich bei größeren Patienten $(>185 \mathrm{~cm}$ ) sollte eine Transplantatlänge von mindestens $70 \mathrm{~mm}$ angestrebt werden. Die GT-Sehne wird, wenn möglich, geschont. Bei schlanken Patienten, insbesondere $\mathrm{Pa}$ tientinnen, kann sie bei einer posterolateralen Instabilität als Transplantat für die posterolaterale Stabilisierung verwendet werden.

Bei einer medialen Instabilität und gleichzeitig gerader oder valgischer Beinachse sollten die Hamstringsehnen der betroffenen Seite geschont werden
(Abb. 16). Als Alternativtransplantat bietet sich das mittlere Drittel der Patellarsehne oder die ST-Sehne der Gegenseite an. Eine chronische mediale Instabilität kann durch Entnahme der ST-Sehne in ein dekompensiertes Stadium überführt werden, das äußerst schwierig zu therapieren ist.

\section{Fixationstechnik}

BTB-Transplantate weisen an beiden Enden einen Knochenblock auf, der im angelegten Bohrkanal fixiert werden muss. Dies gelingt am einfachsten mit einer Schraube (Interferenzschraube), die in den Spalt zwischen Knochenblock und Kanalwand eingedreht wird. Früher wurden Titanschrauben verwendet. Heute hat sich zunehmend die Verwendung von bioresorbierbaren Interferenzschrauben durchgesetzt. Alternativ besteht auch die Möglichkeit einer Verankerung in der Press-fit-Technik [49]. Hierbei wird ein größer dimensionierter Knochenblock entnommen und nach exakter Präparation in den femoralen Kanal eingeschlagen. Der Vorteil liegt in der implantatfreien Verankerung. Nachteilig ist die Entnahme größer dimensionierter Knochenblöcke, sodass mit einer erhöhten Entnahmemorbidität (s. oben) zu rechnen ist.

Der Knochen-Knochen-Fixation steht die Sehnen-Knochen-Fixation gegenüber. Diese ist erforderlich, wenn ein Weichteiltransplantat, das aus einer oder mehreren Sehnen besteht, im Bohrkanal fixiert werden muss. Da diese Transplantate keine Knochenblöcke aufweisen, ist die Transplantatpräparation und die Fixation aufwendiger.

In Abhängigkeit des Fixationsorts sind verschiedene Verankerungskonzepte $\mathrm{zu}$ unterscheiden. Bei einem sehr langen Transplantat kann dieses außerhalb des Gelenks (extraartikuläre Verankerung) direkt im metaphysären Tibia- bzw. Femurbereich am Knochen fixiert werden. Wenn ein sehr langes Transplantat erforderlich ist, müssen hierfür immer STund GT-Sehne entnommen werden. Verwendet man dagegen Fadenmaterial oder vorgefertigte Schlaufen an den Transplantatenden, kann auch ein kürzeres Transplantat derart fixiert werden. Hierbei wird das Fadenmaterial (linkage material) am Knochen fixiert (indirekte Fixation). Typisches Beispiel ist die Verankerung mit einem femoralen und tibialen Fixationsknopf bzw. Fixationsbutton (s. unten). 
In Abhängigkeit vom Fixationsort ist eine semianatomische von einer anatomischen Fixation zu unterscheiden. Bei der semianatomischen Fixation erfolgt die Fixation nicht auf Höhe des Gelenkniveaus, sondern z.B. im Bohrkanal. Typische Beispiele sind die Transfixationssysteme und die tibiale Interferenzschraubenfixation bei der BTB-Technik.

Bei einer anatomischen Verankerung erfolgt die Fixation sehr gelenknah. Typisches Beispiel ist die femorale Fixation mit einer Interferenzschraube. Auch im tibialen Kanal lässt sich die Schraube bis zum Gelenkspalt vordrehen, wenn z.B. ein Weichteiltransplantat verwendet wird.

In früheren Zeiten wurden Fixationssysteme einzig und allein nach der Ausreißkraft beurteilt. So beschäftigten sich zahlreiche Studien mit der Ermittlung der Ausreißkraft unter maximaler Krafteinwirkung und zyklischer Bewegung. Die höchsten Ausreißkräfte zeigten sich bei den Transfixationssystemen. Somit wurden diese nach dem Motto „viel hilft viel“ von der Industrie angepriesen. Es muss jedoch die kritische Frage erlaubt sein: Wieviel Fixationskraft wird benötigt? und Ist eine hohe Fixationskraft bei der femoralen Fixation sinnvoll?

Die Spongiosa im Femur ist im Vergleich zum Tibiakopf wesentlich härter. Damit erklärt sich, dass bei identischer Fixationstechnik die Fixations- bzw. Ausreißkräfte im femoralen Bereich größer sind als tibial. Mit verschiedenen Transfixationssystemen wurden in experimentellen Untersuchungen Ausreißkräfte über $1500 \mathrm{~N}$ ermittelt. Dennoch garantiert eine extrem hohe maximale Ausreißkraft, wie bei den Transfixationssystemen, keinesfalls eine optimale knöcherne Transplantateinheilung. Femoral treten große Winkelbewegungen des Transplantats gegenüber der Bohrkanalwand auf. Daher kann bei unzureichender biologischer Fixation Flüssigkeit in den Spalt zwischen Transplantat und Bohrkanalwand einströmen und damit zu ausgedehnten Kanalerweiterungen führen, was insbesondere bei Transfixationssystemen, der alleinigen femoralen Buttonfixation und bei Verwendung überdimensionierter Interferenzschrauben $\mathrm{zu}$ beobachten ist [13, $19,25,35,96]$. Im femoralen Kanal bestehen demnach mehr biologische, im tibialen Kanal sind dagegen mehr mechanische Probleme, da hier die mecha-

Tab. 3 Vor- und Nachteile bei der Fixation mit dem sog. Fixationsbutton (z. B. EndoButton [Fa. Smith \& Nephew], Flipptack [Fa. Karl Storz], Suture plate [Fa. B. Braun]).

Vorteile
- reduzierte Transplantatlänge
- einfache Anwendung
- Belassen der Grazilissehne (wenn ST-Sehne > $26 \mathrm{~cm}$ )
- keine störenden Implantate bei der Revision
- Variabilität bei der femoralen Bohrkanalplatzierung (transtibiale und anteromediale
- Technik)
- geeignet für HKB-Rekonstruktion
Nachteile
- langes Gesamtkonstrukt (viskoplastische Deformation, geringe Steifigkeit)
- indirekte Verankerung (linkage material)
- aufwendige Transplantatvorbereitung
- präzise Bohrkanalkalibrierung (0,5-mm-Schritte) notwendig
- erhöhtes Risiko der Kanalerweiterung (vermehrte Transplantat-Tunnel-Bewegungen)

Tab. 4 Vor- und Nachteile bei der Fixation mit Interferenzschrauben.

Vorteile
- einfache Transplantatvorbereitung
- Variabilität bei der femoralen Bohrkanalplatzierung (transtibiale und anteromediale
Technik)
- weniger präzise Bohrkanalkalibrierung (1-mm-Schritte) notwendig
- gelenknahe, anatomische Transplantatverankerung
- einfache Anwendung
- Verhinderung des synovialen Einstroms in den Bohrkanal
- Neutralisierung von Transplantat-Tunnel-Bewegungen
- Korrekturmöglichkeit der Transplantatposition durch Position der Schraube
- geeignet für Revisionschirurgie
- geeignet für HKB-Rekonstruktion
Nachteile
- längeres Transplantat erforderlich
- bei Revisionseingriff störendes Implantat (Metall-Interferenzschraube)
- Gefahr der Transplantatrotation und -schädigung bei der Schraubenapplikation
- Probleme der Verankerungsfestigkeit bei geringer Knochendichte
- Gefahr des dorsalen femoralen Tunnelausbruchs
- mechanische Überbelastung des Transplantats bei nicht optimaler Tunnelposition

nischen Grundparameter bedingt durch die weiche tibiale Spongiosa schwieriger sind. Die Spongiosa kann selbst bei jungen Sportlern extrem weich sein.

Die häufigsten Fixationstechniken sind Fixationsbuttons (Fixationsknöpfe), Interferenzschrauben sowie die Transfixationssysteme. Jede dieser Fixationstechniken weist spezifische Vor- und Nachteile auf (Tab. 3 bis $\mathbf{5}$ ). Demnach gilt es auch im Hinblick auf das Operationsspektrum abzuwägen, welche Fixationstechnik verwendet wird. Werden neben VKB- und HKB-Rekonstruktionen auch zahlreiche Revisionen durchgeführt, empfiehlt sich eine Fixationstechnik, mit der sämtliche Probleme zu lösen sind.
Bioresorbierbare Fixationsimplantate In den letzten Jahren hat sich zunehmend die Fixation mit bioresorbierbaren Fixationsmaterialien (Schrauben, Pins) bewährt. Folgende Anforderungen sind zu stellen:

1. Komplette Resorption. Die Materialien sollten nach 1-2 Jahren resorbiert sein. Eine sehr schnelle Resorption innerhalb einiger Monate ist nicht anzustreben, da dies zu Fremdkörperreaktionen führen könnte. Schrauben aus einem einfachen Polylaktid (PLLA) sind hoch kristallin und hydrophob. Daher ist eine Resorption in den ersten 3-4 Jahren kaum zu beobachten. Von uns wurden Schrauben nach 4-6 Jahren ohne die geringsten Zeichen einer Resorption entfernt. Bei der Entfernung von PLLA-Schrauben 
Tab. 5 Vor- und Nachteile bei der Fixation mit Transfixationssystemen (z. B. TransFix [Fa. Arthrex, Karlsfeld], RigidFix ${ }^{\mathrm{TM}}$ [Fa. Mitek, Norderstedt], Bone Mulch Screw ${ }^{\mathrm{TM}}$ [Fa. Biomet, Berlin]).

\begin{tabular}{l} 
Vorteile \\
- einfache Transplantatvorbereitung \\
- extrem hohe initiale Verankerungsfestigkeit \\
- einfache Anwendung \\
\hline Nachteile \\
\hline - grundsätzlich langes Transplantat erforderlich (zusätzliche Entnahme der GT-Sehne) \\
- keine gelenknahe Transplantatverankerung \\
- keine Neutralisierung von Transplantat-Tunnel-Bewegungen \\
- Risiko von extremen Tunnelaufweitungen (besonders bei Transfix) \\
- femorale Tunnelposition nicht korrigierbar (meist transtibiale Technik) \\
- aufwendiges femorales Zielinstrumentarium \\
- hohes Verletzungsrisiko der lateralen Strukturen (Popliteussehne etc.) bei anteromedialer \\
Portaltechnik \\
- Verletzungsrisiko des medialen Seitenbands bei zu weit medialem Beginn des tibialen \\
Bohrkanals \\
- extrem schwierig und aufwendig bei Revisionen (Metall-Pins sehr schwierig zu entfernen, \\
oft erhebliche Tunnelaufweitungen, Röntgendurchleuchtung muss möglich sein) \\
- laterale Inzision erforderlich (erhöhte Morbidität, Traktusirritation) \\
- starker synovialer Einstrom in den Spalt zwischen Transplantat und Bohrkanal (Tunnel- \\
erweiterungen) \\
- mechanische Überbelastung des Transplantats bei nicht optimaler Tunnelposition \\
- eingeschränkte Anwendung bei Revisionen \\
- nicht geeignet für HKB-Rekonstruktion
\end{tabular}

ist zudem festzustellen, dass sich zwischen Schraube und umgebenden Knochen eine „matschige“ Weichteilschicht ausgebildet hat, die eine komplette Abtrennung zwischen Schraubenkörper und Knochen darstellt. Bei der Verwendung von bioresorbierbaren Fixationsimplantaten sollte auf das verwendete Material geachtet werden. Wird ein amorphes Stereokopolymer (z.B. Poly-L-co-D,L-Laktid 70:30) - auch kurz als PLDLLA bezeichnet - verwendet, ist mit einer kompletten Resorption nach 14-20 Monaten zu rechnen.

2. Knöcherner Ersatz des Implantatlagers. Nach kompletter Resorption sollte das Implantatlager knöchern ersetzt sein.

3. Ausreichende Verankerungsfestigkeit. Zahlreiche Studien konnten belegen, dass resorbierbare Interferenzschrauben eine ähnliche Verankerungsfestigkeit aufweisen wie Titanschrauben. Nicht die Interferenzschraube ist die mechanische Schwachstelle, sondern die umgebende Spongiosa im Bohrkanal. Dies gilt insbesondere für den tibialen Kanal.

\section{Ausreichende Torsionsfestigkeit.} Bioresorbierbare Materialien weisen eine geringere Torsionsfestigkeit auf als Metall. Daher müssen Material, Schraubendesign und die Form des Antriebes bei bioresorbierbaren Schrauben optimal aufeinander abgestimmt sein. Nur so ist auch bei har- ten knöchernen Verhältnissen, z.B. beim jungen Patienten, eine ausreichende Antriebskraft gewährleistet. Die erste Generation resorbierbarer Schrauben besaßen meist eine Kopie von Antriebssystemen, wie sie bei Metallschrauben angewendet wurden. Hiermit ließ sich meist nur ein Drehmoment von maximal 4-5 Nm erzielen. Die Torsionsfestigkeit wird zudem wesentlich vom Schraubendesign und der Passform des Antriebssystems bestimmt. Bioresorbierbare Materialien weisen eine reduzierte Biegestabilität bei zyklischer Belastung auf. Daher muss bei bioresorbierbaren Transfixationsstiften und -stäben mit einer deutlich erhöhten Bruchrate gerechnet werden (Abb. 20).

5. Gewindedesign. Das Schraubengewinde sollte so konstruiert sein, dass es einerseits beim Eindrehen das Transplantatgewebe nicht lädiert, andererseits eine ausreichende Fixationskraft gewährleistet ist. Scharfkantige Gewindegänge können in das Transplantatgewebe einschneiden. Schrauben mit sehr stumpfen Gewinde starten dagegen sehr schlecht. So sind bei einem derartigen Schraubentyp sehr hohe Antriebskräfte erforderlich, was wiederum einen extrem stabilen Antrieb erfordert. Die ideale Kombination besteht aus 2 unterschiedlichen Gewindetypen (Abb.21a).
6. Gute Biokompatibilität. Die Schraube sollte aus einem Material beschaffen sein, das eine gute Biokompatibilität besitzt. Eine Trennschicht zwischen Schraube und Bohrkanal wie bei PLLA-Schrauben muss unbedingt vermieden werden, um die Resorption nicht zu beeinträchtigen.

Bei Verwendung großlumiger Schrauben, z.B. bei einem Durchmesser von mehr als $8 \mathrm{~mm}$, besteht das Problem, dass der Knochen nur unzureichend in das Schraubenlumen einwachsen kann. Es stehen nur die Öffnungen des Antriebs und die Öffnung im Bereich der Schraubenspitze als „Eintrittsorte“ zur Verfügung. Daher wurde eine perforierte Interferenzschraube entwickelt [88]. Durch die Perforationen kann Knochen in den Schraubenkörper einwachsen. Hierdurch wird ein 3-dimensionales Durchwachsen des gesamten Schraubenkörpers erreicht, wie tierexperimentell nachgewiesen werden konnte (Abb. 21 b). Diese perforierten Schrauben erfreuen sich zunehmender Verbreitung, da sie auch hinsichtlich der Torsionsstabilität nahezu gleiche mechanische Eigenschaften aufweisen wie unperforierte Schrauben der gleichen Größe [88].

In neuester Zeit werden auch Schrauben und Fixationsstifte aus einer Mischung von Knochenersatzstoff und bioresorbierbarem Grundstoff verwendet. Als Knochenersatzstoff hat sich Trikalziumphosphat (TCP) bei diesen sog. Composite-Implantaten bewährt [88] (Abb. 22). Bezüglich der Anforderungen gilt Gleiches wie bei den bioresorbierbaren Implantaten (s. oben).

\section{Hybridfixation}

Da keine Fixationstechnik nur Vorteile, sondern auch spezifische Nachteile aufweist, gewinnt die Hybridfixation zur femoralen und tibialen Fixation zunehmende Verbreitung. Hierbei werden die Vorteile der jeweiligen Fixationstechnik genutzt und deren Schwächen vermieden. Meist wird eine extrakortikale mit einer anatomischen Verankerungstechnik kombiniert. Gerade bei der Fixation von Weichteilimplantaten bietet sich dieses Vorgehen an, da sich biologische und biomechanische Vorteile ergeben. Eine hohe initiale Verankerungsfestigkeit wird durch die indirekte extrakortikale Verankerung erreicht [95]. Dieser Verankerungstyp weist jedoch Nachteile im Hinblick auf die gelenknahe Fixation auf. Daher wird gleichzeitig zur ge- 

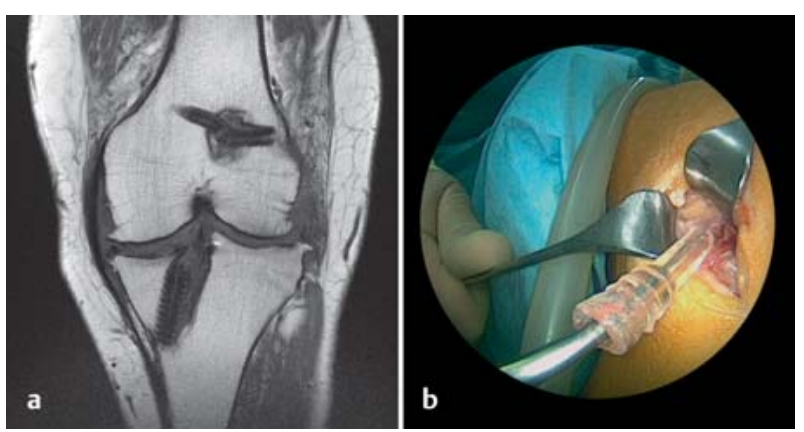

Abb. 20 a und b

Gebrochener Transfixationsstift. MRT-Befund (a), Entfernung eines gebrochenen Transfixationsstifts über eine laterale Inzision. Der Stift hat zur Irritation des Tractus iliotibialis geführt (b).
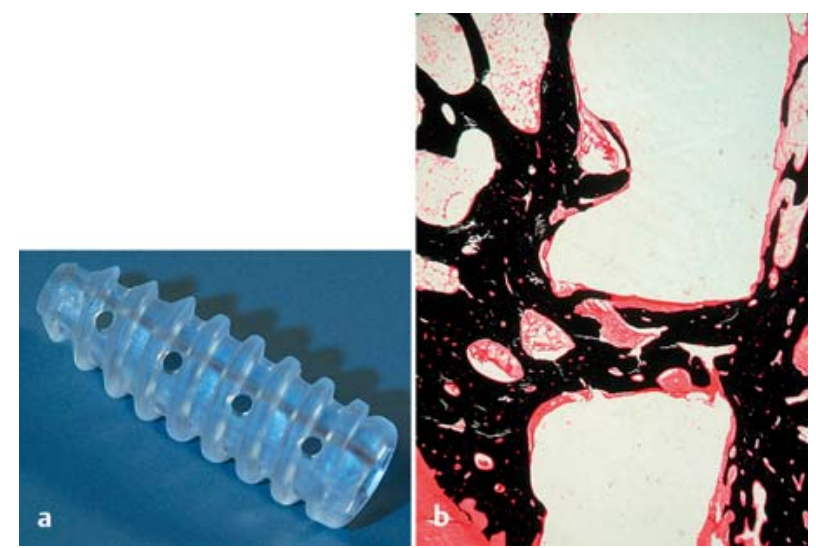

Abb. $21 \mathrm{a}$ und $\mathrm{b}$

Perforierte Interferenzschraube mit scharfen Gewindegängen an der Spitze und stumpfen Gewindegängen am Schraubenkörper (Megafix-P, Fa. Karl Storz, Tuttlingen) (a). Die histologische Untersuchung zeigt ein knöchernes Durchwachsen der Perforation und ein Einwachsen von Knochen in das Schraubenlumen (b).

lenkfernen Fixation eine kleine unterdimensionierte Interferenzschraube gelenknah eingebracht, um das Transplantat an die Bohrkanalwand zu pressen und eine Konstruktlockerung (Slippage) zu minimieren. Untersuchungen haben gezeigt, dass unter dem Ausschluss von Scherkräften eine direkte Sehnen-Knochen-Heilung möglich wird [32,93].

Bei der Hybridfixation ist die Verwendung von überdimensionierten Schrauben nicht mehr erforderlich. Auch heute noch werden von einigen Operateuren Interferenzschrauben mit einem Durchmesser von 10, 11 und sogar $12 \mathrm{~mm}$ zur tibialen Fixation verwendet. Derartig große Schrauben können durch das sehr hohe Drehmoment beim Eindrehen zur Transplantatschädigung führen. Zudem bestehen Probleme, wenn eine Revision, z.B. wegen einer Reruptur des Transplantats, erforderlich wird.

\section{Platzierung der Bohrkanäle}

Die Platzierung des femoralen und tibialen Bohrkanals ist der wichtigste Faktor zur Erzielung eines optimalen Operationsergebnisses $[9,26,28,36,40,46,48$, 78]. Zahlreiche Studien zeigten, dass bei einer neu aufgetretenen Instabilität in den meisten Fällen eine falsche oder suboptimale Bohrkanalposition vorlag $[78,98]$. In früherer Zeit waren 2 Hauptprobleme zu finden:

1. Femoraler Bohrkanal zu weit anterior. Da zur femoralen Fixation häufig eine Metall-Interferenzschraube verwendet wurde, fürchtete der Operateur ein posteriores Ausbrechen des femoralen Bohrkanals. In diesem Fall hätte er keine Möglichkeit mehr gehabt, das Transplantat adäquat zu fixieren. Daher wurde der femorale Kanal, schon aus Sicherheitsgründen, etwas weiter nach anterior platziert (Abb. 23). Zudem wurde die Over-theTop-Position nicht eindeutig dargestellt bzw. war durch eine inadäquate arthroskopische Technik optisch nur sehr schwierig zu evaluieren.

2. Tibialer Bohrkanal zu weit anterior. Die sehr anteriore Platzierung des tibialen Bohrkanals führte in einem sehr hohen Prozentsatz bei Verwendung der BTB-Technik zu Streckdefiziten $[26-28,36,53,67]$. Daher wurde das Hauptaugenmerk auf eine mehr posteriore Tunnelplatzierung, die Anspannung des Transplantats in Streckstellung und ein möglichst frühzeitiges Erreichen der vollen Streckung in der Nachbehandlung gelegt.

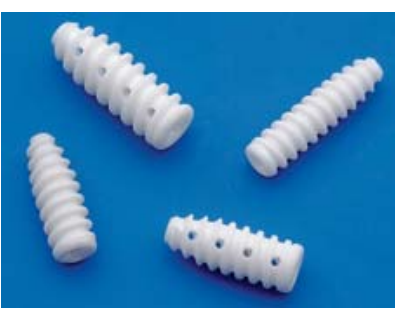

Abb. 22 Unperforierte und perforierte Composite-Schrauben aus einer Mischung von Beta-TCP und PLDLLA (z. B. Megafix C und CP, Fa. Karl Storz, Tuttlingen).

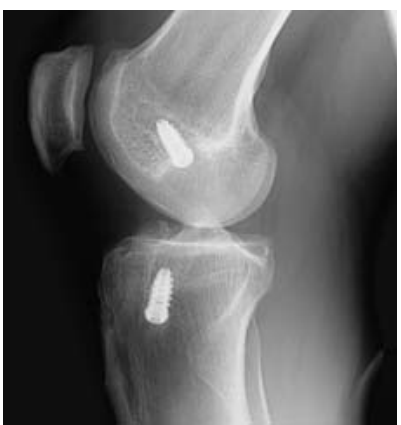

Abb. 23 Extrem anteriore Position des femoralen Kanals.

Unter Verwendung der Weichteiltransplantate (ST- und GT-Sehne) hat sich die rein arthroskopische Technik in den letzten Jahren zunehmend verbreitet. Bei den meisten Techniken wird der femorale Kanal durch den zuvor angelegten tibialen Bohrkanal platziert. Hierzu wird das femorale Zielgerät durch den tibialen Kanal vorgeschoben [5,48, $96,98]$. Vielfach war es dann schwierig, die Over-the-Top-Position zu erreichen. Um diese Position mit dem Zielgerät überhaupt erreichen zu können, war es notwendig, den tibialen Kanal sehr weit posterior anzulegen. Dies kam den Forderungen von Howell et al. [26-28] entgegen, der in zahlreichen Publikationen ein Transplantatimpingement bedingt durch einen zu anterioren Bohrkanal als Ursache eines Transplantatversagens bzw. eines Streckdefizits beschrieb. Die Lage des tibialen Bohrkanals rückte damit sehr weit nach posterior.

Bei den sehr verbreiteten Transfixationstechniken wird der femorale Kanal nach Vorgabe der Hersteller ebenfalls über den tibialen Kanal angelegt. Hierzu wird das femorale Zielgerät durch den tibialen Kanal eingeführt [11]. Da die Zielgeräte aber einen sehr großen Durchmesser aufweisen, muss der Ope- 


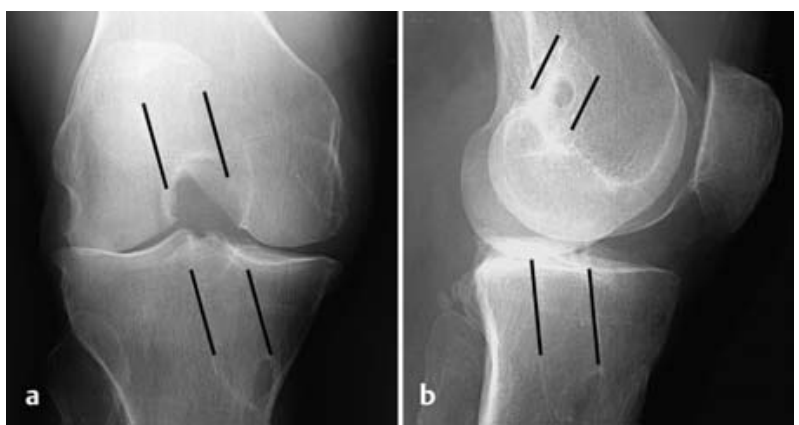

Abb. 24a und b VKB-Rekonstruktion und femorale Fixation mit einem Transfixationssystem (Transfix). Im p.-a. Strahlengang (Rosenberg-Technik) zeigt sich eine deutliche Verschmälerung des medialen und lateralen Gelenkspalts. Gleichzeitig besteht eine massive Erweiterung des tibialen und femoralen Kanals. Der femorale Kanal liegt nahe der High-Noon-Position (a). Im seitlichen Strahlengang lässt sich die Erweiterung des tibialen Kanals, der sehr weit posterior positioniert wurde, deutlich erkennen (b).
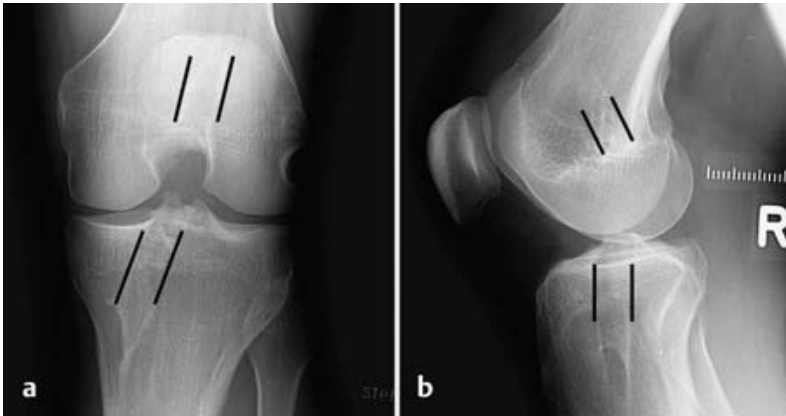

Abb. 25a und b VKB-Rekonstruktion und femorale Fixation mit der Transfixationstechnik. Der femorale Kanal liegt in der HighNoon-Position (a). Auch im seitlichen Strahlengang zeigt sich, dass mit dem tibialen Kanal sehr weit medial begonnen wurde, um bei der transtibialen Vorgehensweise den Femur in adäquater Position zu erreichen. Dies hat zur Irritation des Innenbands bei diesem Patienten geführt. Zudem liegt der tibiale Bohrkanal zu weit posterior (b). rateur den tibialen Kanal sehr weit posterior platzieren, um mit dem Zielgerät überhaupt an die Hinterkante des lateralen Femurkondylus zu gelangen (Abb. 24). Beginnt der tibiale Kanal nicht weit medial, gelangt man mit dem transtibialen Zielgerät sehr leicht in eine hohe Position (sog. High-NoonPosition) (zwischen 11.00-Uhr- und 1.00-Uhr-Position). Daher finden sich bei Verwendung von Transfixationstechniken häufig sehr hohe femorale Tunnel, die mechanisch äußerst problematisch sind (s. unten). Wird mit dieser Technik der femorale Kanal über den medialen Instrumentenzugang angelegt, muss mit einer erhöhten Inzidenz von Knorpelschäden gerechnet werden, wie experimentelle Studien zeigten [11] sowie mit Läsionen lateraler und posterolateraler Strukturen.

Die femorale Transplantatfixation in oder nahe der High-Noon-Position, bei gleichzeitig sehr weit posterior platziertem tibialen Kanal, führt zu einem sehr stabilen Transplantat, insbesondere wenn eine Transfixationstechnik verwendet wurde (Abb.25). Femur und Tibia werden bei dieser Fixationstechnik axial aufeinander gepresst. Daher ist die AP-Laxität (vordere und hintere Schublade) manchmal sogar geringer als am intakten Gelenk. Zahlreiche Patienten sind mit dieser Situation aber nicht zufrieden. Sie beklagen ein permanentes Druckgefühl im Gelenk, manche beschreiben trotz der Stabilität ein Wegknicken oder Wegdrehen des Gelenks. Dies liegt in der unzureichenden Rotationsstabilisierung begründet. Es zeigt sich nicht selten ein einfach positiver

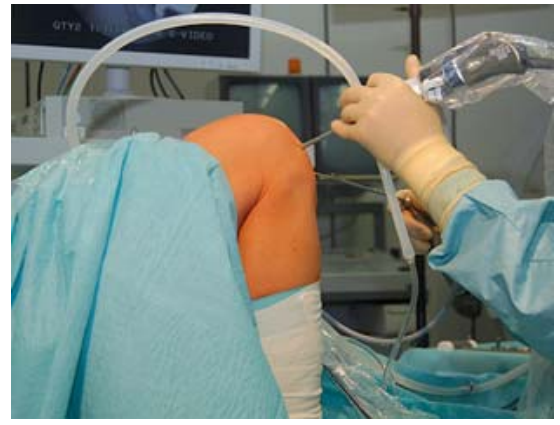

Abb. 26 Anlage des femoralen Bohrkanals in $120^{\circ}$ Flexion über den medialen Instrumentenzugang.

Pivot-Shift-Test. Diese unzureichende Rotationsstabilisierung liegt im Wesentlichen in der Positionierung des femoralen Bohrkanals begründet $[13,98$, 102]. Aus dieser unzureichenden Rotationsstabilisierung wird unter anderem auch die Begründung für die VKBDoppelbündel-Rekonstruktion abgeleitet (s. unten).

Heute sind im Gegensatz zu früher 2 wesentliche Probleme der Tunnelposition zu diskutieren:

1. Tibialer Kanal zu weit posterior

2. Femoraler Kanal zu steil, d.h. nahe der High-Noon-Position (12.00-UhrPosition)

Schon aus diesen Gründen ist eine differenzierte Analyse der Anlagetechnik der Bohrkanäle erforderlich. Die transtibiale femorale Kanalanlage wird heute zunehmend verlassen und durch die Anlage über den medialen Instrumentenzugang ersetzt.

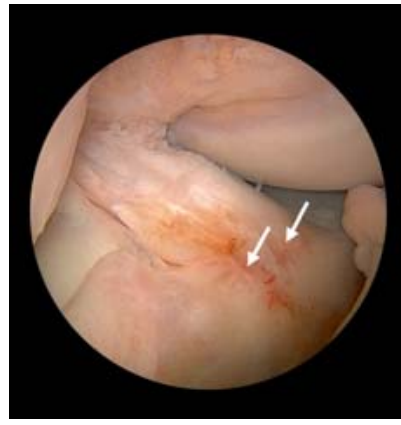

Abb. 27 Inspektion der tibialen VKB-Insertion. Die Insertion reicht sehr weit nach anterior, fast bis zur Tibiavorderkante (Pfeile).

\section{Femoraler Kanal}

Die Positionierung des femoralen Bohrkanals ist wesentlich für den isometrischen Transplantatverlauf verantwortlich. Zur Orientierung wird die Fossa intercondylaris bzw. die Notch nach dem Uhrzeiger eingeteilt. Hierbei ist für die Position des femoralen Kanals der Bereich von 9.00 Uhr bis $12.00 \mathrm{Uhr}$ beim rechten Kniegelenk und von 12.00 Uhr bis 3.00 Uhr beim linken Kniegelenk von Bedeutung. Bei transtibialer Platzierung des femoralen Bohrkanals gelangt man oft in die Nähe der High-Noon-Position (12.00-Uhr-Lage). Bei der Anlage des femoralen Kanals durch den medialen Instrumentenzugang und gleichzeitig einer Beugung von $120^{\circ}$, kann der femorale Kanal dagegen leicht zwischen 9.00 Uhr und 10.00 Uhr bzw. zwischen 2.00 Uhr und 3.00 Uhr angelegt werden (Abb. 26). Zudem bietet die Anlage des Bohrkanals über den medialen Zugang zusätzlich die Möglichkeit, dass dessen Position leicht korrigiert werden kann. 

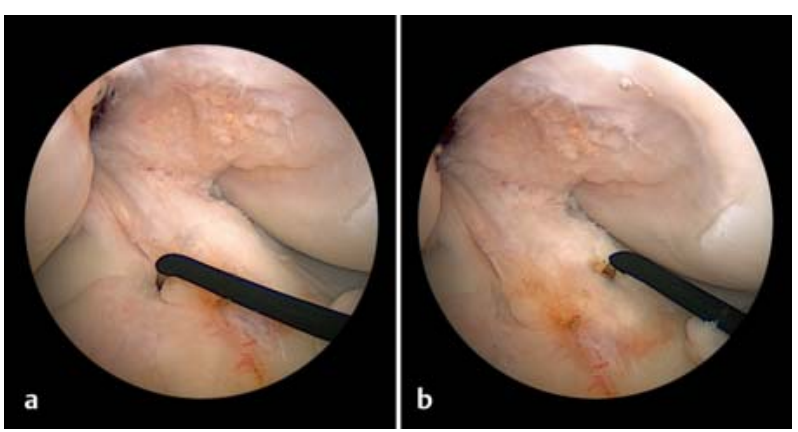

Abb. 28a und $\mathbf{b}$ Orientierung zur Platzierung des tibialen Bohrkanals. Mit dem HF-Messer oder dem Tasthaken wird der hintere Rand des Außenmeniskusvorderhorns palpiert (a) und auf der Verlängerungslinie in Richtung medialer Femurkondylus der Zentrumspunkt des Bohrkanals markiert (b).
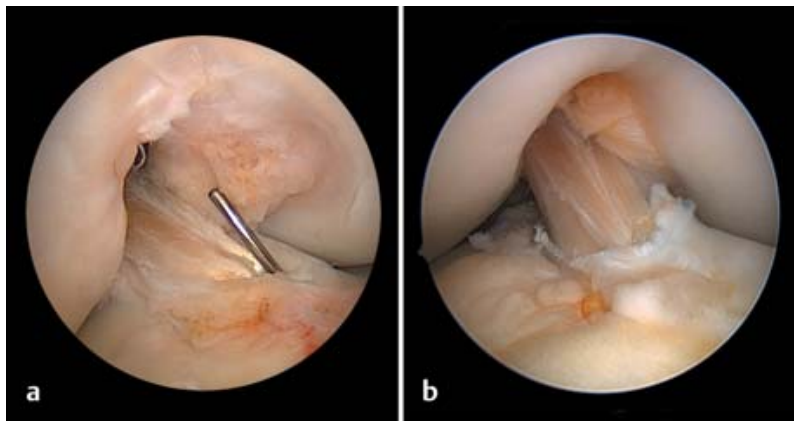

Abb. $\mathbf{3 0}$ a und $\mathbf{b}$ Transplantatverlauf. Nach Einzug des Transplantats zeigt sich, dass die Ausrichtung des tibialen Kanals anders ist als der Transplantatverlauf. Der K-Draht zielt in Richtung des Notchdaches und nicht in Richtung des femoralen Bohrkanals (a). Bei Streckung findet sich kein Transplantatimpingement (b).

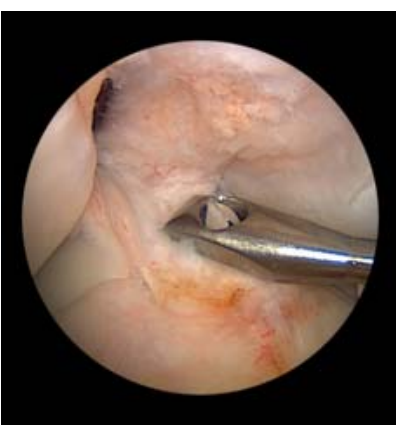

Abb. 29 Einsetzen des Zielgeräts und Vorbohren des Zieldrahts. Dieser erreicht den markierten Zentrumspunkt.
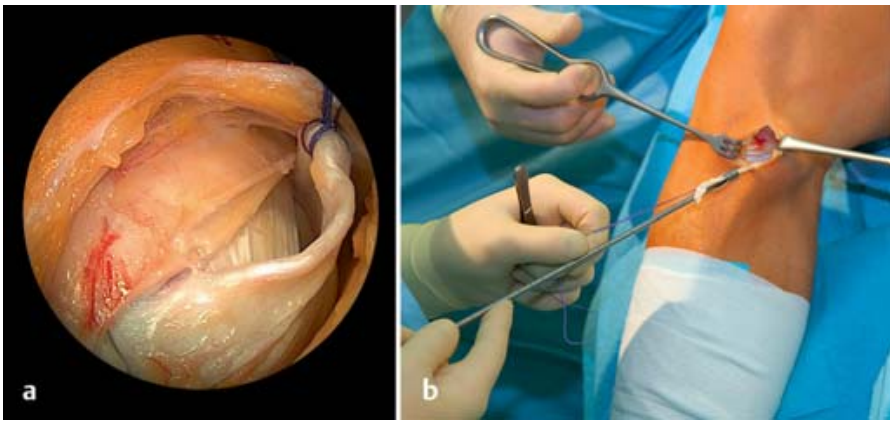

Abb.31 a und b Anschlingen der ST-Sehne mit einem Faden (a). Anschließend wird die tibiale Insertion abgelöst und die Sehne mit dem Sehnenstripper entnommen (b).

\section{Tibialer Bohrkanal}

Zur Platzierung des tibialen Bohrkanals bieten sich verschiedene Orientierungspunkte an. Der alte VKB-Stumpf dient als ideale Positionshilfe. Wurde dieser jedoch bei der Vorarthroskopie entfernt, ist die Orientierung schwieriger. Orientiert man sich an den posterioren VKBFasern oder an der anterioren HKB-Zirkumferenz, wie in früheren Jahren üblich, wird der tibiale Bohrkanal eher zu weit posterior platziert. Einen Eindruck, wie weit anterior das VKB wirklich inseriert, erhält man dann, wenn man ein intaktes Kniegelenk sowohl in Beugung als auch in Streckung arthroskopisch untersucht (Abb. 27). Es ist hier immer wieder erstaunlich, wie weit anterior die tibiale VKB-Insertion liegt. Als wichtige Orientierungshilfe für den tibialen Kanal dient die Verlängerung der Hinterkante des Außenmeniskusvorderhorns. Verlängert man diese Linie nach medial, liegt auf ihr der Zentrumspunkt des tibialen Bohrkanals (Abb. 28). Diese Bohrkanalposition erscheint relativ anterior, insbesondere wenn man die transtibiale Anlage des femoralen Kanals ge- wohnt ist (Abb. 29). Führt man einen K-Draht durch einen derart angelegten tibialen Bohrkanal, gelangt man meist nicht in die Region des zuvor angelegten femoralen Bohrkanals. Der in dieser Position durchgeführte Impingement-Test lässt ebenfalls befürchten, dass es $\mathrm{zu}$ einem massiven Transplantatimpingement kommt. Dies findet sich aber in der Regel nicht, da das Transplantat nicht in Verlängerung des tibialen Bohrkanals, sondern nach lateral kaudal verläuft, um den femoralen Bohrkanal zu erreichen (Abb.30a). Streckt man das Knie nach dem Transplantateinzug, zeigt sich trotz des relativ weit anterioren tibialen Bohrkanals kein Impingement (Abb. 30 b).

Einbündel- versus Doppelbündeltechnik

Das VKB besteht anatomisch aus einem anteromedialen und posterolateralen Bündel [12,39,61,62,102]. Es sind zahlreiche experimentelle und klinische Studien bekannt, die die Einbündel- und Doppelbündeltechnik miteinander vergleichen $[1,3,39,80,100]$. Die postulier- ten Vorteile der Doppelbündeltechnik sind die verbesserte Stabilität, die bessere Sicherung gegen Rotationskräfte sowie eine geringere Rate an pathologischen Pivot-Shift-Tests. Dies konnte in zahlreichen Studien belegt werden [39, 100].

Demgegenüber steht ein zeitlich erhöhter Aufwand bei der Operation, möglicherweise eine erhöhte Komplikationsrate mit Arthrofibrosen, Bewegungseinschränkungen und algodystrophen Reaktionen, sowie ein erhöhter Bedarf an Implantaten.

Bei der viel zitierten Studie von Yasuda et al. [100] wurde eine Einbündeltechnik mit einer nicht anatomischen und einer anatomischen Doppelbündeltechnik verglichen. Als Ergebnis berichten diese Autoren, dass bei der Einbündeltechnik 50\%! der operierten Patienten einen einfach- oder zweifach positiven Pivot-Shift-Test aufwiesen. Dieser Prozentsatz ist in der Gruppe mit der anatomischen Doppelbündeltechnik deutlich geringer. Es ist jedoch festzustellen, dass 
diese Einbündeltechnik in einer Art erfolgte, die heute kaum mehr praktiziert wird bzw. werden sollte (alleinige femorale Fixation mit Fixationsbutton, High-Noon-Position des femoralen Bohrkanals, alleinige extraartikuläre tibiale Fixation). Derartige Ergebnisse würde wohl kein Referent auf einem Kongress präsentieren, sodass man sich fragt, wie die Ergebnisse der Autoren vor der Zeit der Doppelbündeltechnik waren.

Der Vergleich zwischen Einbündel- und Doppelbündeltechniken ist nur dann sinnvoll und wissenschaftlich eindeutig zu bewerten, wenn die Einbündeltechnik in einer Technik durchgeführt wird, die den aktuellen Anforderungen an die Tunnelpositionierung und Transplantatfixation entspricht. In anderen Studien wurde kein Unterschied zwischen Doppel- und Einbündeltechnik nachgewiesen [80].

Kriterien, bei denen eine Doppelbündeltechnik möglicherweise sinnvoll ist, müssen noch eindeutig herausgearbeitet werden. Es ist sicherlich nicht jeder Patient für eine Doppelkanaltechnik geeignet, insbesondere wenn es sich um sehr kleine Patienten oder kleine Gelenke handelt. Andererseits sind bei chronischen Instabilitäten und großen Patienten mit ausgedehnten Rotationsinstabilitäten durchaus Doppelbündeltechniken zu erwägen. Bis der definitive Vorteil in wissenschaftlichen Studien hinreichend belegt ist, sollte die Einbündeltechnik als Standard gelten.

\section{Spezielle Operationstechnik}

Im Folgenden wird die vom Autor verwendete Operationstechnik beschrieben. Modifikationen sind in jeglicher Richtung möglich. Die beschriebene Technik hat sich seit Jahren im klinischen Alltag bewährt.

\section{Transplantatentnahme}

Zur Transplantatentnahme wird ein Längsschnitt medial und etwas distal der Tuberositas platziert. Palpatorisch wird der Bereich des Pes anserinus identifiziert. Die zunächst zu palpierende Sehne entspricht meist nicht der STSehne, sondern der GT-Sehne. Distal der tastbaren GT-Sehne wird die Faszie des Pes anserinus in Längsrichtung inzidiert. Distal der Inzision lässt sich dann die STSehne auffinden (Abb.31a). Die Sehne wird mit dem Sehnenstripper entnommen (Abb.31 b). Ihre Länge beträgt

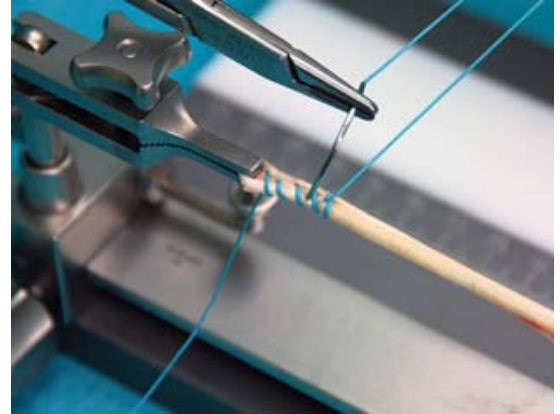

Abb.32 Armierung der Sehnenenden auf dem Sehnenboard.
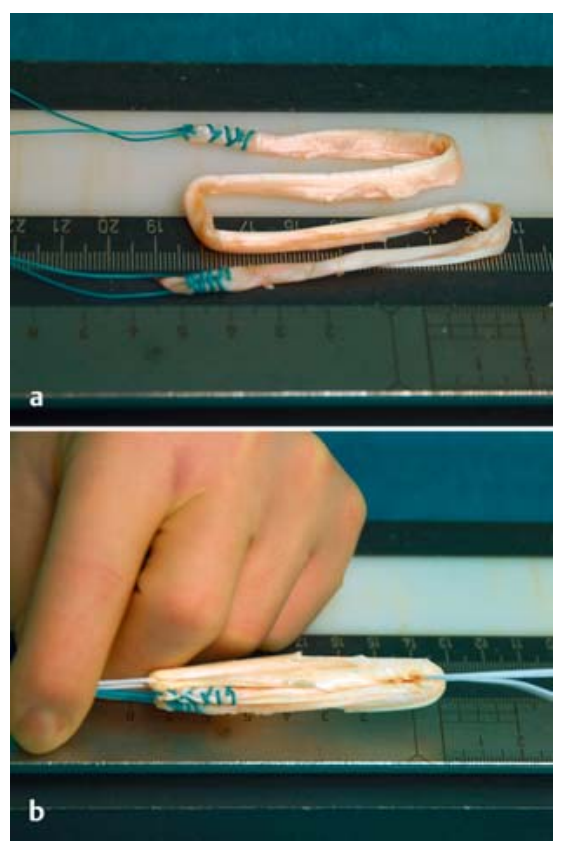

Abb.33a und b Transplantatvorbereitung. Legen der ST-Sehne als sog. M (a). In dieser Form wird die Sehne in die Doppelschlaufe gelegt (b).

durchschnittlich 26 bis $28 \mathrm{~mm}$, die der GT-Sehne 20 bis $22 \mathrm{~cm}$. Das weitere Vorgehen hängt im Wesentlichen von der Fixationstechnik ab.

Bei der Transplantatentnahme wird darauf geachtet, dass die GT-Sehne geschont wird. Die alleinige Verwendung der ST-Sehne reicht bei über $90 \%$ der Patienten aus. Besitzt der Patient eine sehr kurze ST-Sehne $(<24 \mathrm{~cm})$ oder liegt ein VKB-Revisionseingriff vor, wird die GTSehne entnommen.

\section{Transplantatvorbereitung}

Die ST-Sehne wird von Muskelgewebe befreit. Überstehende instabile Anteile werden auch im Bereich der Sehnen-

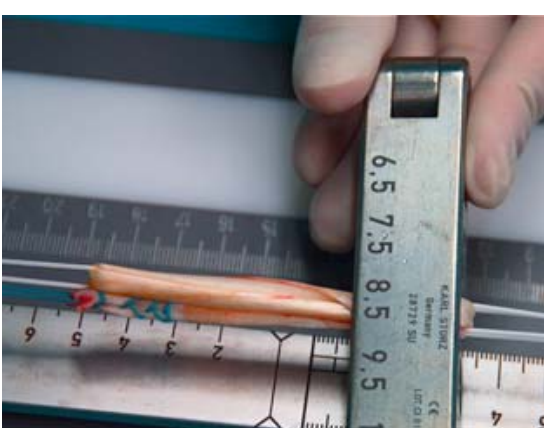

Abb. 34 Bestimmung des Transplantatdurchmessers mit dem Sehnenstärketester.

enden entfernt. Die Sehne wird dann in die Sehnenhalter des Sehnenboards eingespannt und mit Fixationsnähten an jeder Seite fixiert (z.B. PremiCron, Stärke 2, Fa. B. Braun, Melsungen) (Abb. 32). Die Sehne wird in der M-Technik präpariert, sodass sie nicht durchtrennt werden muss. Damit wird nicht nur die Transplantatvorbereitung vereinfacht, sondern auch das Linkage-Material reduziert (Abb. 33). Nach Einspannen des Transplantats auf dem Sehnenboard wird der Transplantatdurchmesser bestimmt (Abb.34). Insbesondere im femoralen Bereich wird auf eine sehr präzise Bestimmung des Durchmessers geachtet. Die Bestimmung des Durchmessers erfolgt auf $0,5 \mathrm{~mm}$ genau.

\section{Femoraler Bohrkanal (Abb. 35)}

Nach Anlage des hohen anterolateralen Arthroskopzugangs erfolgt die Inspektion des Gelenks. Zur Anlage des femoralen Bohrkanals wird ein tiefer medialer Instrumentenzugang platziert. Eine eventuell vorhandene Plica infrapatellaris und instabile VKB-Reste werden entfernt. Dann wird das femorale Zielgerät durch den tiefen medialen Instrumentenzugang eingeführt und das Kniegelenk auf mindestens $120^{\circ}$ gebeugt. In dieser Position ist sehr leicht die 2.00-3.00-Uhr- bzw. 9.00-10.00-UhrPosition zu erreichen. Korrekturen in dieser Position sind sehr einfach, da das Zielgerät nur durch die Weichteile des medialen Instrumentenzugangs in seinen Bewegungen limitiert wird.

Bei zunehmender Beugung wird der anteriore Raum durch das Eindringen des Hoffa-Fettkörpers in die Fossa intercondylaris verkleinert. Trotzdem sollte man immer versuchen, eine ausreichende Übersicht zu behalten. Der angelegte Bohrkanal liegt weit posterior in einer 9.30-Uhr-Position (Abb. 36). 

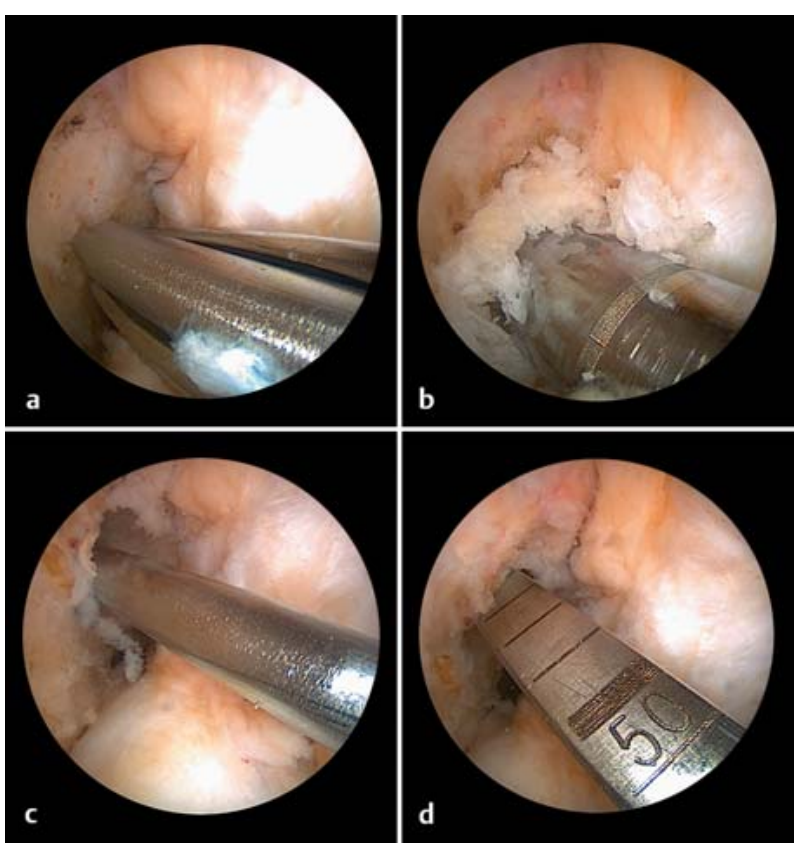

Abb. 35 a bis d Platzierung des femoralen Bohrkanals. Einführen des Zielgeräts und Vorbohren eines K-Drahts, bis die laterale Femurkortikalis perforiert ist (a). Dann wird das Zielgerät zurückgezogen und der Draht mit einem Kopfbohrer überbohrt. Dieser weist den gleichen Durchmesser wie der femorale

Transplantatanteil auf. Ein Durchbrechen der lateralen Femurkortikalis muss vermieden werden (b). Nach Zurückziehen des Kopfbohrers (c) wird der Draht mit einem 4,5-mmBohrer überbohrt und die laterale Femurkortikalis durchbrochen. AbschlieBend erfolgt die Längenmessung des Bohrkanals. Es ergibt sich hier eine Länge von $42 \mathrm{~mm}$ (d).

\section{Tibialer Bohrkanal}

Nach Anlage des femoralen Bohrkanals wird das Kniegelenk in eine Flexion von ca. $50^{\circ}$ zurückgelagert. In dieser Position erhält man einen sehr guten Überblick über die tibiale VKB-Insertion. Es hat sich bewährt, zunächst den Zentrumspunkt des tibialen Kanals zu markieren (zur Lokalisation des Kanals s. Abb. 28 bis 30 ).

Ist das Zielgerät platziert, wird der Bohrdraht vorgebohrt. Die Länge des tibialen Bohrkanals kann am Zielgerät abgelesen werden und sollte nicht weniger als $40 \mathrm{~mm}$ betragen. Im Normalfall liegt sie zwischen 45 und $50 \mathrm{~mm}$. Der Bohrdraht wird zuerst mit einem 6-mm-Bohrer überbohrt. Führt man dann wieder einen Bohrdraht ein oder den stumpfen Trokar, zeigt sich, dass es oft in Verlängerung des tibialen Bohrkanals zu einem Impingement mit dem Dach der Fossa intercondylaris kommt (Abb. 37). Das Transplantat verläuft jedoch nicht in Verlängerung des tibialen Kanals, sondern in Richtung des zuvor angelegten femoralen Kanals (s. unten).

Dann wird erneut ein K-Draht eingeführt und mit dem Kopfbohrer auf den definitiven Transplantatdurchmesser erweitert. Der Durchmesser des tibialen Kanals beträgt meist $1 \mathrm{~mm}$ mehr als der femorale Kanal.

\section{Transplantatpräparation}

Da die Transplantatlänge meist zwischen 65 und $70 \mathrm{~mm}$ beträgt, ist eine optimale Ausrichtung des Transplantats auf den femoralen Kanal, die intraartikuläre Strecke und den tibialen Kanal Voraussetzung für die optimale Fixation. Die intraartikuläre Transplantatstrecke beträgt ca. 25 bis $27 \mathrm{~mm}$. Das Transplantat sollte femoral mindestens $16 \mathrm{~mm}$ eingezogen werden. Sämtliche Strecken, wie die Länge des Transplantats im femoralen Kanal, die intraartikuläre Transplantatlänge, aber auch die Länge des Transplantats im tibialen Kanal, sind auf der Skala des verwendeten Sehnenboards einfach abzulesen. Die Einziehtiefe des Transplantats in den femoralen Kanal lässt sich durch Anziehen der kräftigen Fäden der Doppelschlaufe (Ethibond $1 \mathrm{~mm}$ ) leicht kontrollieren. Hat das femorale Transplantatende die gewünschte Einziehtiefe erreicht, wird der Ethibondfaden mehrfach geknotet. Abschließend erfolgt die Armierung des Transplantats mit einem Vicrylfaden (2-0) im Bereich des femoralen und tibialen Anteils. Auf dem Transplantat wird zum Abschluss noch eine Flippmarkierung aufgebracht (Abb. 38).

\section{Transplantateinzug und femorale Fixation}

Zunächst wird ein Bohrdraht mit Öse und eingelegter Fadenschlaufe bei $120^{\circ}$ Flexion durch den tiefen medialen In-

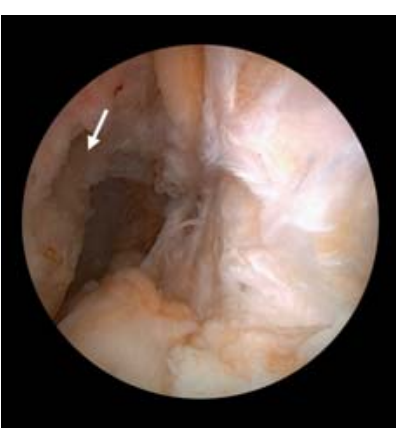

Abb.36 Femoraler Bohrkanal sehr weit posterior lokalisiert (Pfeil).

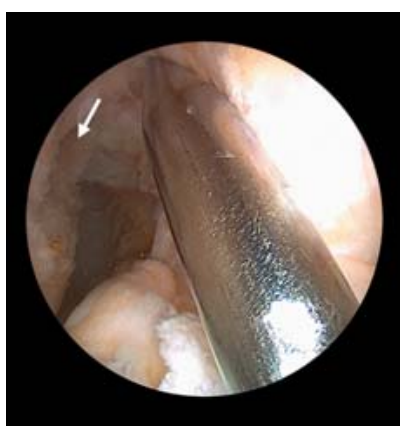

Abb. 37 Der durch den tibialen Kanal eingeführte stumpfe Trokar erreicht das Dach der Fossa und nicht den Eingang des femoralen Kanals, der wesentlich weiter posterior lateral (Pfeil) liegt.

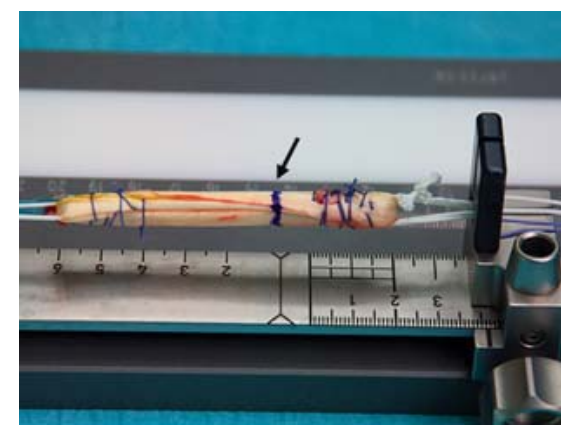

Abb. 38 Eingespanntes Transplantat. Der Transplantatanteil, der in den femoralen Kanal eingezogen wurde, wurde mit zirkulären Fäden armiert. Eine Flippmarkierung (Pfeil) wurde aufgebracht.

strumentenzugang in den femoralen Kanal eingeführt. Dann wird die Fadenschlaufe mit einer Fadenfasszange, die durch den tibialen Kanal eingeführt wird, durch den tibialen Kanal ausgezogen. Wird das Kniegelenk abwechselnd gestreckt und gebeugt, zeigt sich, dass der Transplantatverlauf dem Fadenverlauf entspricht und nicht der Verlängerung des tibialen Bohrkanals (Abb. 39). In die Fadenschlaufe werden dann der Zug- und Flipp-Faden des Fixationsbut- 

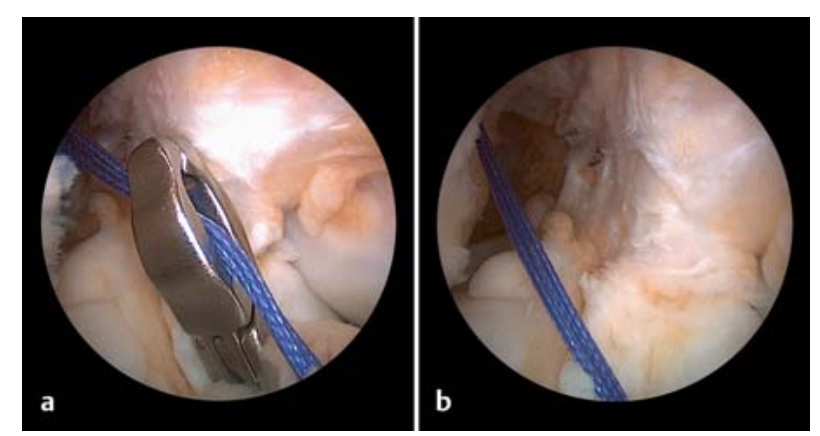

Abb.39a und b Einzug des Durchzugsfadens. Der Durchzugsfaden wurde über den medialen Instrumentenzugang in den femoralen Kanal eingeführt. Mit der Fadenfasszange wird er durch den tibialen Kanal ausgezogen.
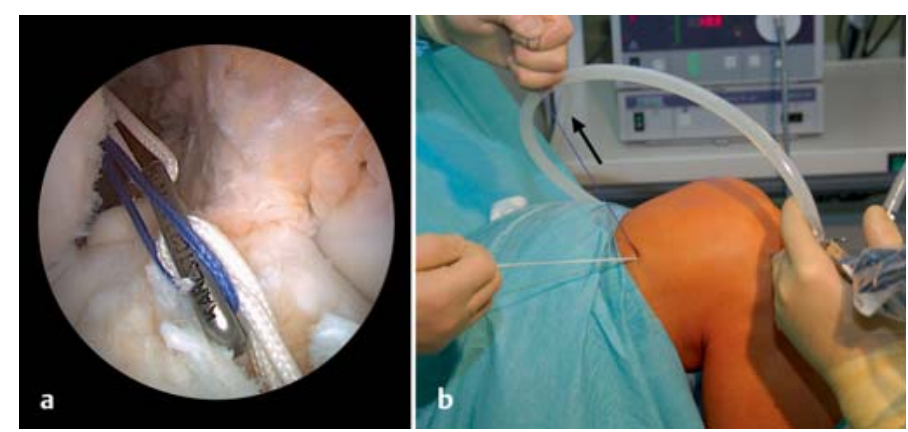

Abb. 40 a und $\mathbf{b}$ Transplantateinzug. Erscheint der Fixationsbutton im Gelenk, wird noch einmal seine Stellung kontrolliert. Der Zugfaden (weiß) muss führen (a). Flippen des Buttons durch Zug am Flippfaden (Pfeil), wenn die Flippmarkierung auf dem Transplantat den Eingang des femoralen Kanals erreicht hat $(\mathbf{b})$.
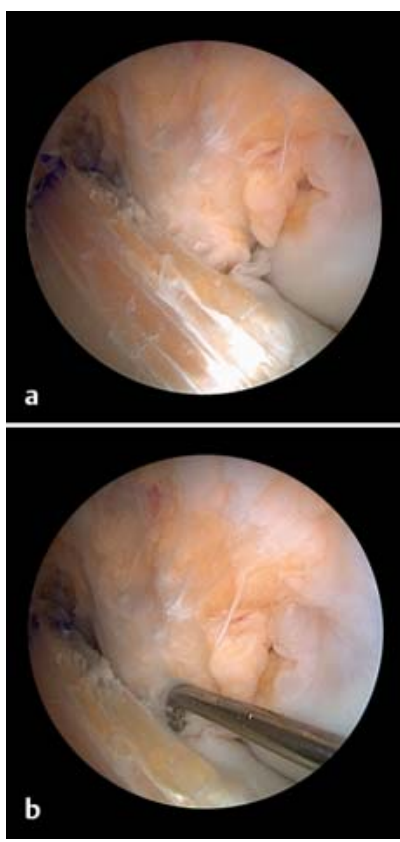

Abb.41a und b Transplantatverlauf. Das VKB-Transplantat verläuft sehr flach in Richtung des femoralen Bohrkanals (a). Es kommt auch zu keinem Impingement zwischen VKB und HKB. Der Spalt ist mit dem Tasthaken leicht zu verifizieren (b).
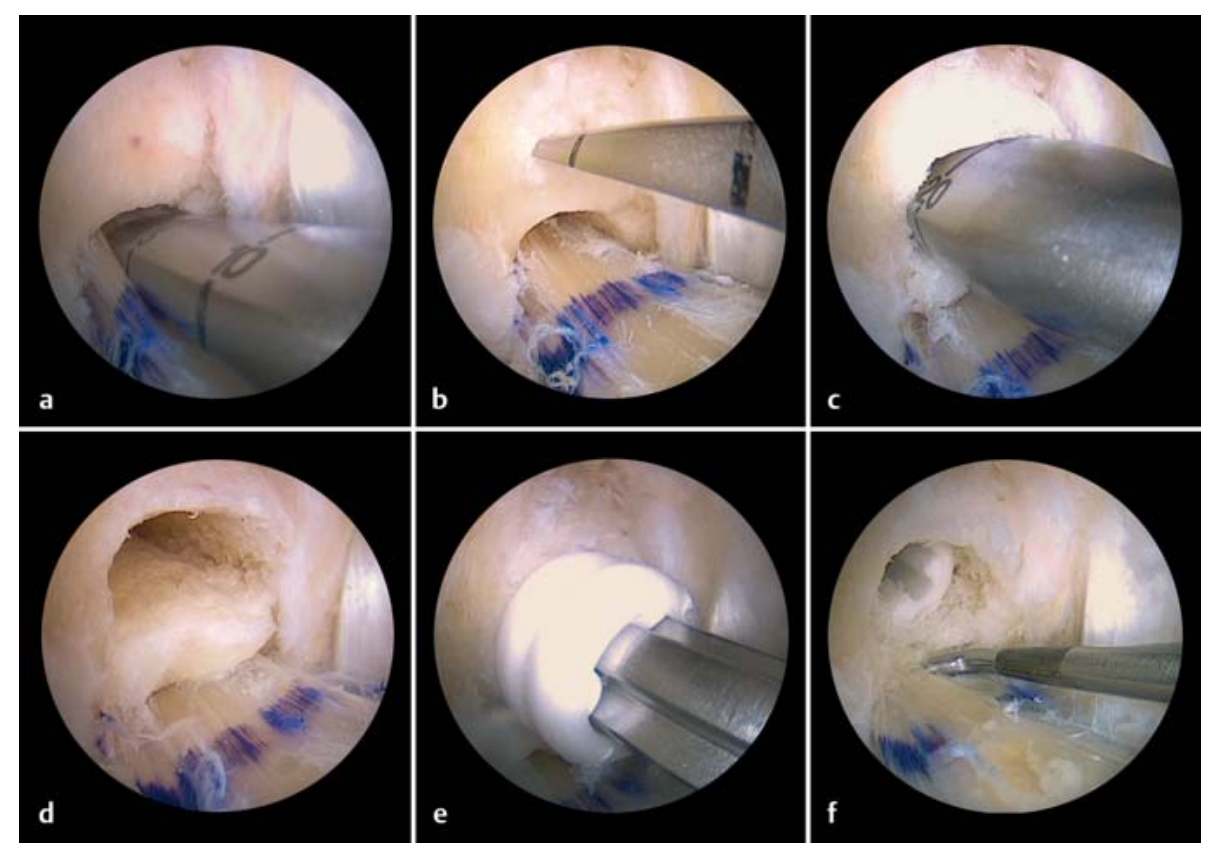

Abb. 42 a bis f Femorale Bone-Wedge-Technik. Wird das Transplantat mit dem Meißel nach unten gedrückt, lässt sich der Spalt zwischen Transplantat und Bohrkanal leicht darstellen (a). Etwa 3 bis $4 \mathrm{~mm}$ oberhalb des Bohrkanals wird der Meißel bis auf eine Tiefe von $20 \mathrm{~mm}$ eingeschlagen (b,c). Durch leichte Rotation des Meißels wird eine Knochenschuppe mobilisiert (d) und in den entstandenen Spalt eine kleine bioresorbierbare Interferenzschraube oder wie hier eine Composite-Interferenzschraube (Megafix C, Fa. Karl Storz, Tuttlingen) eingedreht (e). Der Schraubenkopf wird $2 \mathrm{~mm}$ unter das Kortikalisniveau versenkt und abschließend mit dem Tasthaken der enge Kontakt von Transplantat und Bohrkanalwand überprüft (f). tons eingelegt. Es ist darauf $\mathrm{zu}$ achten, dass der Zugfaden führt (Abb. 40).

Das Transplantat wird bei $120^{\circ}$ gebeugtem Kniegelenk so weit eingezogen, bis die Flippmarkierung den Eingang des femoralen Kanals erreicht. Dann wird der Fixationsbutton geflippt, d.h. es wird am Flippfaden gezogen (Abb. 40 b). Hierunter stellt sich der Fixationsbutton quer, durch Zurückziehen des Transplantats legt sich dieser auf die laterale Femurkortikalis auf. Die Flippmarkierung erscheint wieder im Gelenk. Das
Gelenk wird mehrmals durchbewegt. Der optimale Verlauf des Transplantats zeigt sich auch daran, das kein Impingement mit dem HKB vorliegt (Abb. 41).

Palpiert man den Spalt zwischen Transplantat und femoralem Bohrkanal, zeigt sich, dass dieser mit dem Tasthaken oder auch mit einem stumpfen Trokar zu erweitern ist. Dies lässt auf einen inkompletten Sehnen-Knochen-Kontakt schließen. Es ist nun das Ziel, diesen Spalt zu schließen. Hierzu bietet sich die Bone-Wedge-Technik an (Abb.42).
Diese wird als zusätzliche Fixation verwendet. In den präparierten Spalt wird eine kleine bioresorbierbare oder Composite-Interferenzschraube eingedreht. Bei einem Transplantatdurchmesser von $7 \mathrm{~mm}$ und weniger wird eine $6 \times 19-\mathrm{mm}$ Schraube, bei einem Durchmesser von $8 \mathrm{~mm}$ und mehr genügt eine $7 \times 19-\mathrm{mm}$ Schraube, um eine Anpressung des Transplantats an die Bohrkanalwand zu erzielen. Bei VKB-Revisionen kann eine größer dimensionierte Schraube notwendig sein. Vorteile der Bone-WedgeTechnik sind eine zirkuläre knöcherne 


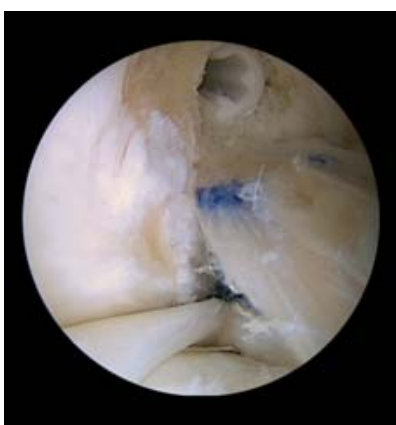

Abb. 43 Die abschließende Inspektion in der Halb-Vier-Position zeigt eine ovaläre Form des Transplantats, ähnlich dem nativen VKB, am Eintritt in den femoralen Bohrkanal. Ein lateraler Scheibenmeniskus findet sich als Nebenbefund.

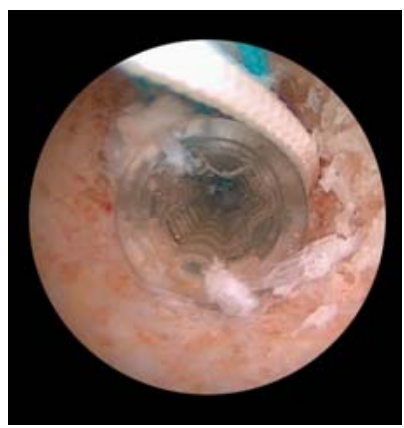

Abb. 44 Kontrolle der Schraubenposition im tibialen Bohrkanal. Die Schraube liegt unterhalb des Transplantats.

Umschließung des Transplantats. Zudem kommt es nicht zur Rotation des Transplantats wie bei einer Interferenzschraube, die direkt in den Spalt zwischen Transplantat und Bohrkanalwand eingedreht wird. Zudem lässt sich eine mehr ovaläre Transplantatkonfiguration unmittelbar am Austritt des femoralen Kanals erreichen (Abb. 43). Damit ähnelt der Transplantatdurchmesser dem nativen VKB. Tomihara et al. [90] konnten 2007 in tierexperimentellen Untersuchungen mit einer sehr ähnlichen Fixationstechnik zeigen, dass durch Einbringen eines freien Knochenblocks zwischen Transplantat und Interferenzschraube die maximale Ausreißfestigkeit deutlich erhöht werden konnte. Sie lag deutlich höher als bei einer direkten Fixation mit einer Interferenzschraube. Histologisch zeigte sich ein organisierteres und reiferes Gewebe zwischen Transplantat und Knochenblock als bei der direkten Fixation. Auf diesem Prinzip beruht auch die BoneWedge-Technik.
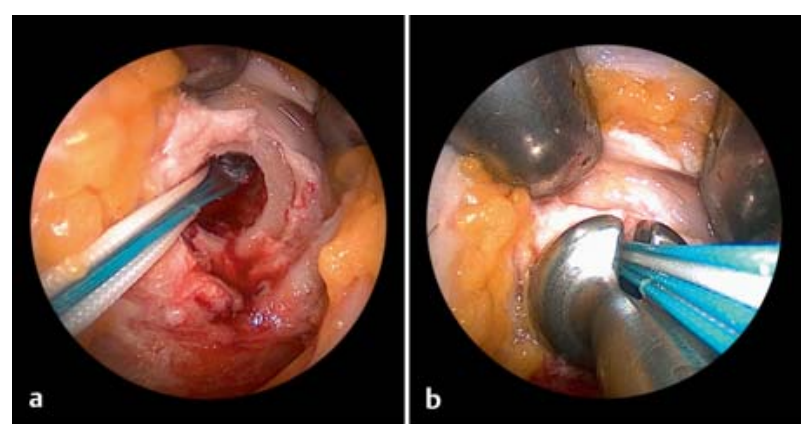

Abb. 45 a bis $\mathbf{d}$ Tibiale Fixation mit Fixationsbutton. Der tibiale Kanaleingang wird von Periost befreit (a). Mit dem Setzgerät erfolgt das zirkuläre Eindrücken der Spongiosa, um ein „Bett“ für den Endotack zu schaffen (b). Dann wird der Endotack (Fa. Karl Storz, Tuttlingen) eingesetzt und mit dem Fadenhal-
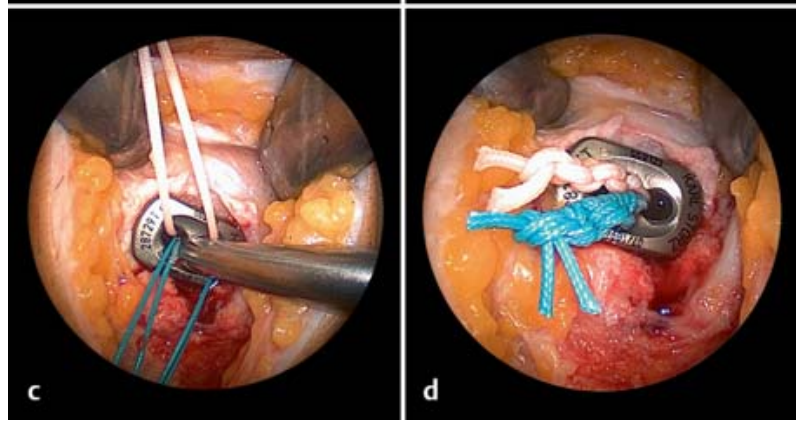
ter fixiert (c). Die Armierungsfäden (grün) werden verknotet, ebenso wie der weiße Faden (Ethibond, Stärke $1 \mathrm{~mm}$ ), der durch die distale Sehnenschlaufe verläuft (d).

Dann werden die tibialen Fixationsfäden angezogen und das Knie mehrmals durchbewegt. Dies führt zu einem Setzen der Fixationsfäden im Transplantat, dient aber auch der Überprüfung eines Impingements.

\section{Tibiale Fixation}

Die tibiale Fixation wird ebenfalls als Hybridfixation vorgenommen. Zunächst erfolgt die gelenknahe Fixation mit einer unterdimensionierten bioresorbierbaren Interferenzschraube. Im tibialen Kanal stehen in der Regel 20 bis $25 \mathrm{~mm}$ Transplantatanteil zur Fixation bereit. Würde man eine alleinige Fixation mit einer bioresorbierbaren Interferenzschraube anstreben, müsste man bei einem tibialen Kanaldurchmesser von z. B. $9 \mathrm{~mm}$ eine überdimensionierte Schraube von 10 oder sogar $11 \mathrm{~mm}$ verwenden. Zudem erscheint der 25-mm-Transplantatanteil sehr kurz, sodass bei derartigen Techniken die zusätzliche Entnahme der GT-Sehne notwendig wäre, um ein längeres Transplantat zu erhalten. Dies soll jedoch vermieden werden. In Sonderfällen kann die GT-Sehne sogar für eine posterolaterale Stabilisierung bei chronischen Instabilitäten verwendet werden. Dies ist sicherlich sinnvoller als die GT-Sehne beim VKB-Ersatz „zu opfern".

In $10^{\circ}$ Flexion erfolgt das Eindrehen der tibialen Interferenzschraube. Bei einem Tunneldurchmesser von 8 oder $9 \mathrm{~mm}$ wird standardmäßig eine $8 \times 23-\mathrm{mm}$ -
Schraube (z. B. Megafix P, Fa. Karl Storz, Tuttlingen) verwendet. In Abhängigkeit der tibialen Transplantatposition wird die Schraube anterior, medial oder lateral oder auch posterior des Transplantats platziert. Hierfür erfolgt zunächst eine Dilatation mit dem stumpfen Trokar. In den Spalt wird ein NitinolDraht eingeführt. Die Schraube wird unter gleichzeitiger manueller Anspannung des Transplantats eingedreht. Somit kann nochmals eine Korrektur der Transplantatposition erreicht werden. Optimal ist die Schraubenpositionierung unterhalb des Transplantats, da hier eine längere Kontaktstrecke zur Verfügung steht (Abb. 44).

Nach Einbringen der Interferenzschraube kann deren Position durch Einführen der Optik in den tibialen Bohrkanal kontrolliert werden (Abb.44). Liegt die Schraube in einer suboptimalen Position, kann sie noch einmal nachkorrigiert werden.

Da diese unterdimensionierte Schraube alleine nicht ausreicht, eine suffiziente Transplantatfixation auch bei zyklischen Belastungen zu gewährleisten, muss das Transplantat noch mit einer 2. Fixation gesichert werden. Hierzu wird der Eintritt des tibialen Bohrkanals vom Periost befreit und mit einem Setzgerät das „Bett“ für den tibialen Button geschaffen. Dieser wird in das Bett gedrückt und die Fixationsfäden des Transplantats verknotet. Mit einem speziellen Schlüssel kann der Fixationsbut- 
ton gegenüber dem Transplantat verdreht werden, was zur Anspannung der Armierungsfäden und damit zur Erhöhung der Fixationskraft führt (Abb. 45).

\section{Nachbehandlung}

Das Kniegelenk wird für 1 Woche in eine $0^{\circ}$-Schiene gelegt, sofern keine anderen intraoperativen Maßnahmen erfolgt sind, die eine modifizierte Nachbehandlung erfordern. Die Schiene bleibt für 1 Woche Tag und Nacht. Aus dieser heraus werden erste Mobilisationsübungen durchgeführt. Die Schiene wird nach der ersten postoperativen Woche für weitere 3 Wochen nachts angelegt. Tagsüber erhält der Patient eine ACLSchiene, z. B. Don-Joy-Fource-Point ACL (Abb.18). Diese wird auf einen Bewegungsbereich von $0-0-90^{\circ}$ limitiert. Die Besonderheit dieser Schiene (ACL FourcePoint, Fa. Ormed.djo, Freiburg) besteht darin, dass durch das speziell konstruierte Scharniergelenk streckungsnah ein Abbremseffekt gegeben ist, der dem natürlichen Abbremsen des Kniegelenks durch die Schlussrotation nachempfunden ist. Hierdurch wird das Gelenk zudem vor einer unkontrollierten Überstreckung geschützt (Abb.18).

Der Patient darf ab dem ersten postoperativen Tag mit halbem Körpergewicht belasten. Diese Belastung kann ab der 3. bis 4. Woche auf Vollbelastung gesteigert werden. Schwellungszustände werden mit Lymphdrainagen behandelt. Die Mobilisation wird zunehmend erhöht, sodass ab der 6 . Woche die intensive Nachbehandlung mit Muskelkräftigung und forcierter Mobilisation beginnen kann.

Der Eintritt der Sportfähigkeit hängt im Wesentlichen vom Zustand des Gelenks, aber auch von der Sportart ab. Man muss sich an dieser Stelle vergegenwärtigen, dass eine Bandruptur nicht nur zu einer mechanischen Störung, d. h. Instabilität führt, sondern dass auch propriozeptive Regelkreise zerstört sind. Selbst wenn das Transplantat das native VKB an Festigkeit und Querschnitt übertrifft, weist es trotzdem den gravierenden Nachteil auf, keine propriozeptive Ankopplung (Schutzreflex) an die Oberschenkelmuskulatur zu besitzen $[6,7]$.

Im Allgemeinen beträgt der Zeitraum zwischen Operation und Eintritt der Sportfähigkeit 6 bis 12 Monate. Gerade Berufssportler drängen auf eine schnelle Wiederaufnahme der Sportfähigkeit.
Bedingt durch die meist bessere und intensivere Rehabilitation ist dies oft auch möglich.

\section{Hinteres Kreuzband}

Verletzungen des hinteren Kreuzbands (HKB-Verletzungen) zählen zu den schwersten Bandverletzungen. Oft werden diese Verletzungen erst spät oder gar nicht diagnostiziert, sodass eine verzögerte oder adäquate Therapie erfolgt [18,23,69,86,88]. Folgen können schwerste, die Gelenkfunktion deutlich beeinträchtigende Störungen, bis hin zur Invalidität der Patienten sein. Nach wie vor bestehen hinsichtlich des therapeutischen Managements auch heute noch erhebliche Kontroversen [85,88]. Dies liegt sicherlich am geringen Wissensstand bei HKB-Verletzungen im Vergleich zu den sehr viel häufigeren VKB-Läsionen. Andererseits wurde eine Vielzahl verschiedener therapeutischer Vorgehensweisen in der Literatur nur in sehr geringen Fallzahlen untersucht, sodass keine klaren Schlussfolgerungen bezüglich des therapeutischen Konzepts abzuleiten sind.

Folgende Einteilung hat sich bewährt:

1. akut (weniger als 3 Wochen)

2. subakut (3 Wochen bis 3 Monate)

3. chronisch (3 Monate bis 5 Jahre)

4. langzeit-chronisch (mehr als 5 Jahre)

\section{Ursache}

Als Hauptursache für eine HKB-Läsion wurde über lange Jahre ein direktes Anpralltrauma wie z.B. eine Armaturenbrettverletzung (Dash Board Injury) verantwortlich gemacht. Hierbei wirkt eine direkt nach posterior gerichtete Kraft auf die proximale Tibia bei gebeugtem Kniegelenk ein [18,23].

Die Analyse unseres Patientenguts zeigte aber, dass sich $42 \%$ der Patienten die HKB-Läsion bei einer sportlichen Aktivität zugezogen haben [69]. Hier ist die häufigste Ursache mit über $21 \%$ beim Fußball zu suchen. Ein Sturz mit gebeugtem Kniegelenk z.B. auf den Boden oder einen am Boden liegenden Gegenspieler führt zu einer nach posterior gerichteten Kraft und in der Folge nicht selten zu einer HKB-Läsion. Überproportional häufig ist der Torwart betroffen, bei dem 2 typische Verletzungsmechanismen anzutreffen sind. Der Torwart steht mit leicht gebeugtem Knie in der „Warteposition“ und der Gegenspieler springt oder fällt gegen das gebeugte
Knie im Bereich der proximalen Tibia. Damit findet ein ähnlicher Krafteinstrom wie bei der Armaturenverletzung statt. Ebenfalls kann er mit gebeugtem Knie gegen den Torpfosten prallen. Auch hierbei wirkt eine nach posterior gerichtete Kraft auf den Tibiakopf. Daher sollte jede Knieverletzung beim Fußballspieler differenzialdiagnostisch hinsichtlich einer HKB-Läsion abgeklärt werden.

Nicht selten treten HKB-Läsionen auch bei Stürzen vom Motorrad oder Fahrrad auf. Seltener sind beidseitige HKB-Läsionen, die jedoch dann differenzialdiagnostisch abgeklärt werden müssen, wenn beide Kniegelenke verletzt sind.

\section{Symptomatik}

\section{Akute Läsion}

Die Symptomatik bei der akuten Läsion ist unterschiedlich und kann von völliger Schmerzfreiheit bis $\mathrm{zu}$ hin $\mathrm{zu}$ massiven Schmerzen im gesamten Gelenk reichen. Manchmal finden sich nur Schmerzen im Bereich der Kniekehle mit einer Unterblutung, wenn die Verletzung einige Tage zurückliegt (Abb. 46). Periphere Bandverletzungen sind, falls sie vorliegen, oft symptomatisch prominent und können $\mathrm{zu}$ sehr ausgedehnten Hämatomen führen (Abb. 47). Oft wird bei akuten Knieverletzungen aber nicht an eine HKB-Läsion gedacht.

\section{Chronische Läsion}

Häufig werden chronische HKB-Läsionen trotz auffälliger Symptomatik nicht adäquat diagnostiziert $[18,23]$. Hauptsymptome sind Instabilität und Schmerzen. Schmerzen treten bevorzugt im medialen Gelenkkompartment auf, sodass häufig an eine Meniskusläsion oder beginnende mediale Kompartmentarthrose gedacht wird. Untersuchungen von Skyhar et al. (1993) zeigen jedoch, dass jede HKB-Läsion zur Druckerhöhung im medialen Kompartment und femoropatellaren Gelenk führt.

Die Instabilitätssymptomatik ist bei den Patienten oft nicht so gravierend ausgeprägt wie bei einer VKB-Insuffizienz. Dies liegt darin begründet, dass die Hauptinstabilität bei einer HKB-Läsion in höheren Flexionsgraden zu finden ist, insbesondere dann, wenn eine isolierte HKB-Läsion vorliegt. Bei kombinierten und komplexen Verletzungen mit Beteiligung des HKB und peripherer Band- 
strukturen, z. B. posterolateralen Strukturen, tritt die Instabilität dagegen auch in extensionsnahen Stellungen auf und ist damit klinisch mehr prominent. Beklagen Patienten eine Instabilität bei gleichzeitig vorliegender HKB-Läsion, muss immer an eine periphere Begleitläsion (z.B. posterolaterale Instabilität) gedacht werden.

\section{Diagnostik}

Die Diagnostik der HKB-Läsion ist nicht einfach. Bei frischen Verletzungen werden derartige Läsionen leicht übersehen, da das Hauptaugenmerk auf andere Verletzungen, z.B. Frakturen gerichtet wird $[23,84]$.

\section{Akute Läsion}

Inspektorisch findet sich oft ein unauffälliger Befund, ein intraartikulärer Erguss ist nicht obligat. Blutungen treten meist in die posterioren Weichteile aus (Abb. 46 und 47). Palpatorisch findet sich häufig ein Druckschmerz in der Kniekehle. Prätibiale Kontusionsmarken und Schürfwunden weisen auf den prätibialen Anprall bei der Verletzung hin.

\section{Chronische Läsion}

Der Untersuchungsbefund bei chronischen Läsionen reicht von einem völlig unauffälligen Kniegelenk, über eine ausgedehnte spontane hintere Schublade bis hin zu massiven arthrotischen Veränderungen (Abb.48). Früher wurde das Zeichen einer spontanen hinteren Schublade als eindeutiger Hinweis auf eine HKB-Läsion gewertet. Dies ist aber nur unzureichend, da bei einer spontanen hinteren Schublade von einer kombinierten Instabilität, d.h. von einer Läsion des HKBs und einer peripheren Struktur, meist der posterolateralen Gelenkecke, auszugehen ist.

Bei jedem Verdacht auf eine HKB-Läsion ist die Prüfung der sog. palpatorischen hinteren Schublade zu empfehlen [84, 88] (Abb.49). Der Untersucher legt seine Hand auf die vordere Kniekontur, wobei Zeige- und Mittelfinger auf der Patella liegen. Die Handinnenfläche wird auf die Tuberositas tibiae gelegt. Ein Zurücksinken der Tuberositas tibiae wird sehr leicht durch die Überstreckung in den Fingergrundgelenken vom Untersucher wahrgenommen. Dieser Test dauert im Seitenvergleich nur we-

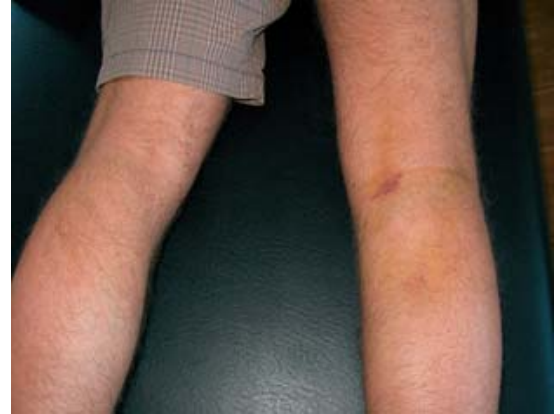

Abb. 46 Hämatom im Bereich der Kniekehle bei HKB-Ruptur (7 Tage alt).

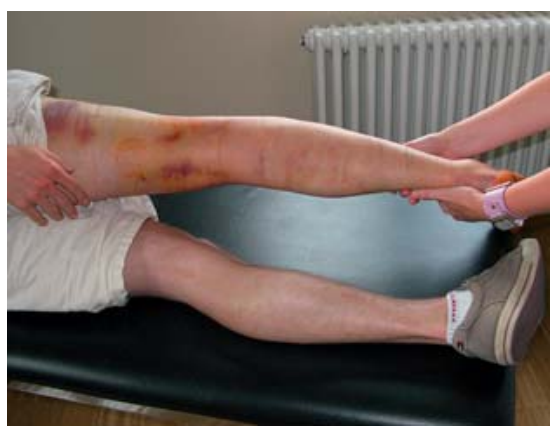

Abb. 47 Komplexe Läsion mit HKB-Ruptur und lateraler Kapselruptur (Varustrauma beim Fußball, 23-jähriger Patient). Ausgedehnte Hämatome im Bereich des Oberschenkels, der Kniekehle, aber auch des Unterschenkels bis zum oberen Sprunggelenk reichend.

nige Sekunden. Findet sich ein Seitenunterschied, wird dies sehr schnell vom Untersucher registriert und muss als Hinweis auf eine HKB-Läsion gewertet werden.

\section{Stabilitätsprüfung}

Die Untersuchung der hinteren Schublade in $90^{\circ}$ Flexion ist der klassische klinische Test. Es werden sowohl das Ausmaß der posterioren Tibiaverschiebung als auch die Qualität des Anschlags beurteilt. Oft findet sich ein gewisser Restanschlag selbst bei sehr ausgeprägter HKB-Insuffizienz.

Die Prüfung der hinteren Schublade erfolgt auch in Innen- und Außenrotation. Findet sich eine ausgeprägte hintere Schublade in Außenrotation, die durch Innenrotation der Tibia reduziert wird, liegt zusätzlich eine posterolaterale Instabilität vor. Findet sich sowohl in Innen- als auch in Außenrotation eine ausgedehnte hintere Schublade, muss von einer posteromedialen Instabilität ausgegangen werden.

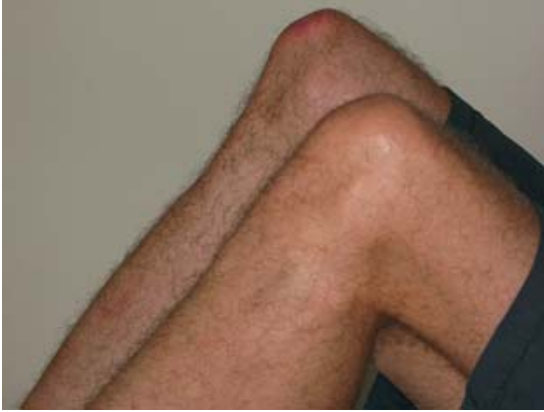

Abb. 48 Spontane hintere Schublade.
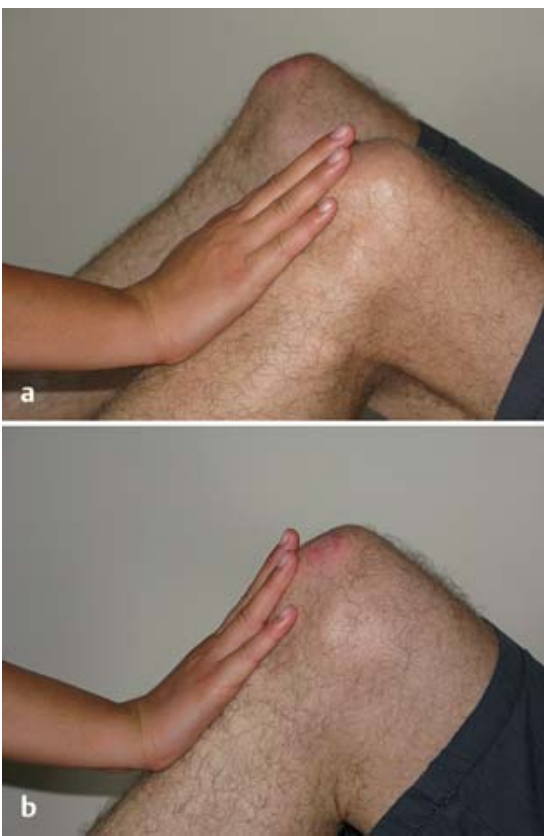

Abb.49a und b Prüfung der „palpatorischen hinteren Schublade“. Der Untersucher legt seine Hand auf die vordere Kniekontur, wobei Zeige- und Mittelfinger auf der Patella liegen. Die Handinnenfläche wird auf die Tuberositas tibiae gelegt. Wird die Hand auf das gesunde Gelenk gelegt, findet sich keine Überstreckung der Fingergrundgelenke (a). Ein Zurücksinken der Tuberositas tibiae wird sehr leicht durch die Überstreckung in den Fingergrundgelenken vom Untersucher wahrgenommen (b).

Bei der Stabilitätsprüfung ist auch der Lachman-Test zu berücksichtigen (Abb.8). Dieser wird bekanntlich bei jeder Verletzung als „VKB-Test“ geprüft. Die Beurteilung des Lachman-Tests bei einer HKB-Insuffizienz ist jedoch problematisch. Der Unterschenkel wird dabei aus einer posterioren Tibiaposition nach anterior gezogen. Dies führt oft zur Interpretation eines deutlich positiven Lachman-Tests mit endgradig festem Anschlag. Als Verdachtsdiagnose wird nicht selten von einer VKB-Elonga- 
tion gesprochen. Da bei diesem Zustand jedoch der Unterschenkel aus einer posterioren Tibiaposition in die Neutralposition gezogen wird, muss bei jeder HKB-Insuffizienz mit einem positiven Lachman-Test mit festem Anschlag gerechnet werden, sofern das VKB intakt ist. Daher muss jeder deutlich positive Lachman-Test mit endgradig festem Anschlag an eine HKB-Läsion denken lassen.

Da das therapeutische Management im Wesentlichen auch durch die Begleitläsionen im Bereich der medialen, dorsomedialen bzw. lateralen und dorsolateralen Kapsel beeinflusst wird, umfasst die Untersuchung auch die Prüfung der medialen und lateralen Instabilität in Streckung und leichter Flexion (Abb.16). Die Außenrotation des Unterschenkels in $30^{\circ}, 60^{\circ}$ und $90^{\circ}$ im Seitenvergleich wird ebenfalls geprüft. Findet sich eine vermehrte Außenrotation, muss mit einer posterolateralen Instabilität gerechnet werden.

\section{Bildgebende Diagnostik}

\section{Röntgen}

Zum Ausschluss einer knöchernen Verletzung erfolgen bei der frischen Verletzung Röntgenaufnahmen in 2 Ebenen. Wenn möglich, erfolgt keine a.-p. Aufnahme, sondern eine p.-a. Aufnahme in der Rosenberg-Technik (s. oben).

Knöcherne Bandausrisse, meist tibial lokalisiert, sind nicht immer eindeutig auf den seitlichen Aufnahmen erkennbar. Besteht der Verdacht einer derartigen Läsion, sollte eine MR-Tomografie oder besser eine Computertomografie erfolgen. Differenzialdiagnostisch gilt es Verkalkungen im HKB-Verlauf, wie sie bei alten Läsionen anzutreffen sind, abzugrenzen.

\section{Gehaltene Röntgenaufnahmen}

Das Ausmaß der posterioren Tibiaverschiebung ist durch die klinische Untersuchung sowohl bei frischen als auch bei chronischen Verletzungen schwierig zu bestimmen $[82,87]$. Testgeräte, wie das KT-1000 (Abb.9), haben sich zur Quantifizierung bei HKB-Läsionen nicht bewährt [24]. Als wichtigste diagnostische Technik muss die gehaltene Röntgenaufnahme angesehen werden.

Bei frischen Verletzungen kann bis zum 14. Tag eine gehaltene Röntgenaufnahme für die hintere Schublade angefertigt werden. Jedoch sollte mit einem dosierten hinteren Schubladenstress von $5 \mathrm{~kg}$ untersucht werden. Nach dieser Zeit sind gehaltene Aufnahmen nicht zu empfehlen, da sie zur Dehnung des sich in Heilung befindlichen HKBs führen können. Die Untersuchung erfolgt im Scheuba-Apparat in $90^{\circ}$ Kniebeugung (Abb. 50). Kann der Patient das Gelenk nicht bis $90^{\circ}$ beugen, sollte die Untersuchung beidseits bei $80^{\circ}$ oder $75^{\circ}$ erfolgen. Bestehen starke Schmerzen, sollte auf die Untersuchung verzichtet werden.

Bei chronischen HKB-Läsionen hat sich die gehaltene Röntgenaufnahme zur Erfassung der posterioren Tibiaverschiebung bewährt und gilt als wichtigstes diagnostisches Verfahren zur Quantifizierung der posterioren Instabilität [24, 88]. Die Untersuchung erfolgt bei $90^{\circ}$ gebeugtem Kniegelenk im ScheubaApparat. Hierbei wird eine gehaltene Röntgenaufnahme für die hintere und vordere Schublade im Seitenvergleich durchgeführt. Es wirkt eine Kraft von jeweils $15 \mathrm{kp}$ ein (Abb. 51). Da sich auch Kinder HKB-Läsionen zuziehen können, wird auch hier die posteriore Instabilität mit gehaltenen Röntgenaufnahmen erfasst (Abb. 52).

\section{Messverfahren}

Die Ausmessung der gehaltenen Röntgenaufnahme erfolgt in der Technik nach Jacobsen $[37,38]$. Hierbei wird durch das mediale Tibiaplateau eine Linie angelegt. Diese sog. Tibiaplateaulinie verläuft durch die anteriorsten und posteriorsten Punkte des Tibiaplateaus. Dann werden die posterioren Begrenzungen des medialen und lateralen Femurkondylus und des medialen und lateralen Tibiaplateaus markiert. Durch den Mittelpunkt zwischen den femoralen Kondylen wird auf der Tibiaplateaulinie eine Senkrechte errichtet. Damit ergibt sich die femorale Referenzlinie. Die tibiale Referenzlinie entsteht durch Bildung einer Senkrechten auf der Tibiaplateaulinie, die durch den Mittelpunkt der dorsalen Begrenzung des medialen und lateralen Tibiaplateaus verläuft. Der Abstand der tibialen und femoralen Referenzlinie ergibt das Ausmaß der Schublade. Liegt die tibiale Referenzlinie posterior der femoralen, ergibt sich ein negativer Schubladenwert. Dies ist meist bei Aufbringen eines hinteren Schubladenstresses der Fall. Der hintere Schubladenwert wird daher als negativer Wert, z.B. - $16 \mathrm{~mm}$ angegeben. Unter einer anterioren Kraftapplikation

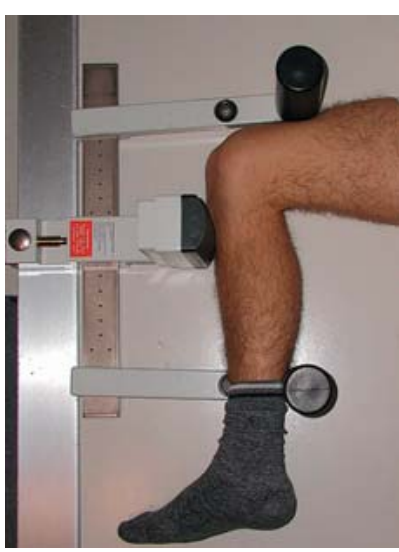

Abb. 50 Testgerät nach Scheuba zur Anfertigung gehaltener Röntgenaufnahmen der hinteren und vorderen Schublade in $90^{\circ}$ Flexion.

kann die tibiale Referenzlinie anterior der femoralen Referenzlinie liegen. Der Schubladenwert wird dann als positiver Wert (z.B. $+6 \mathrm{~mm}$ ) angegeben. Bei einer fixierten hinteren Schublade findet sich trotz des anterioren Schubladenstresses ein negativer Schubladenwert, z.B. - 4 oder -6 mm (Abb. 53).

Finden sich Schubladenwerte von mehr als - 12 bis - $13 \mathrm{~mm}$, muss mit einer peripheren Instabilität gerechnet werden. Leichenexperimentelle Untersuchungen haben gezeigt, dass eine isolierte HKBDurchtrennung zu maximalen Schubladenwerten von - 10 bis - 12 mm führt. Alle Werte darüber müssen den Verdacht auf eine periphere Instabilität aufkommen lassen. Es gilt, besonders dann nach einer posterolateralen und/oder posteromedialen Instabilität zu suchen. Wird diese bei der definitiven Versorgung nicht angegangen, besteht ein hohes Risiko, dass das HKB-Transplantat überlastet und elongiert bzw. insuffizient wird.

\section{Fixierte hintere Schublade}

Es stellt sich die berechtigte Frage, warum auch die vordere Schublade als gehaltene Aufnahme geprüft wird. Gerade bei chronischen Instabilitäten wurde sehr häufig ein Zustand beobachtet, der mit dem Begriff „fixierte“ hintere Schublade beschrieben wird. Hierbei verbleibt die Tibia in einer posterioren Position, obwohl eine nach anterior wirkende Kraft (vordere Schublade) einwirkt. Die Tibia kann nicht in die Neutralstellung gebracht werden. Eigene Untersuchungen zeigten, dass bei bis zu $44 \%$ der Patienten mit einer chronischen HKBInsuffizienz unter der vorderen Schub- 

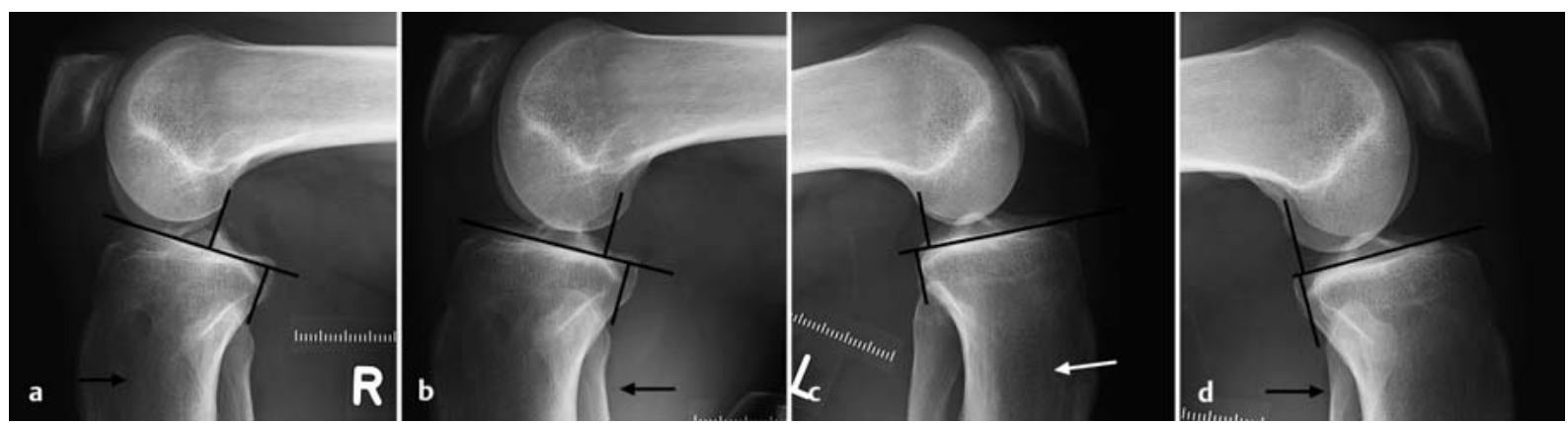

Abb. 51 a bis d American Footballspieler (28 Jahre), Knieverletzung vor 14 Monaten. Im erstbehandelnden Krankenhaus wurde eine VKB- und HKB-Ruptur diagnostiziert. Trotz bekannter HKB-Läsion erfolgte - völlig unverständlicherweise - zuerst eine VKB-Rekonstruktion. Die gehaltene Aufnahme der hinteren Schublade in $90^{\circ}$ Flexion ergibt einen Wert von - 17 mm (a). Die Aufnahme der vorderen Schublade zeigt einen Wert von $-8 \mathrm{~mm}$, somit besteht eine fixierte hintere Schublade. Es ist zudem der sehr weit posterior platziert tibiale Bohrkanal zu erkennen (b). Die Vergleichsaufnahmen der gesunden Gegenseite zeigen eine hintere Schublade von $-3 \mathrm{~mm}$ (c) und einen Tibiawert von - $1 \mathrm{~mm}$ bei der Auslösung der vorderen Schublade (d).
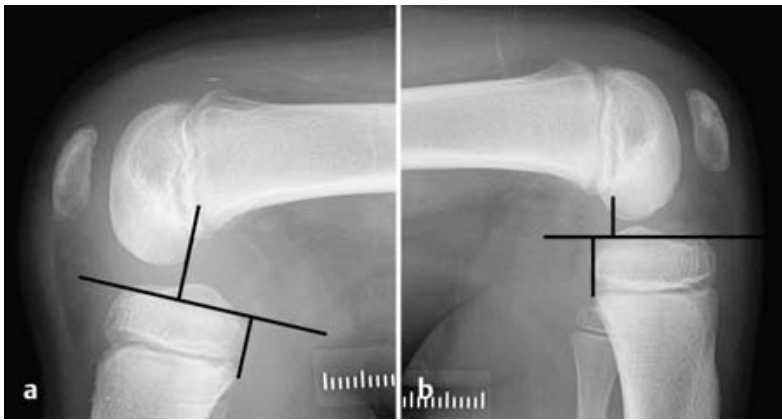

Abb. 52 a und b Massive hintere Schublade bei 8-jährigem Mädchen. Die gehaltene Aufnahme zeigt auf der verletzten - $14 \mathrm{~mm}$ (a) und auf der gesunden Seite $-5 \mathrm{~mm}$ (b).

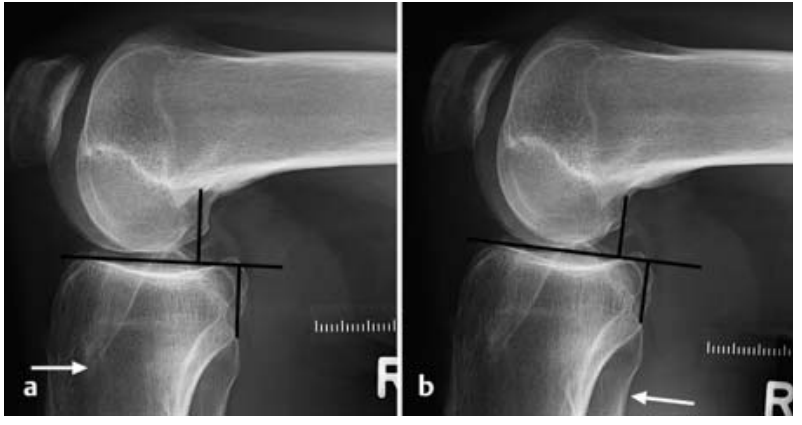

Abb.53a und b Fixierte hintere Schublade. Der Patient klagt 5 Jahre nach VKB-Rekonstruktion über rezidivierende Schmerzen und Schwellungen, insbesondere über ein massives Druckgefühl im Kniegelenk. Die gehaltene Röntgenaufnahme zeigt einen Wert von - $11 \mathrm{~mm}$ für die hintere Schublade als Ausdruck der vorliegenden HKB-Insuffizienz (a). Die vordere Schublade zeigt einen Wert von $-7 \mathrm{~mm}$. Damit liegt eine fixierte hintere Schublade vor. lade keine Neutralstellung der Tibia erreicht werden konnte [86]. Wird in einer derartigen Situation eine HKB-Rekonstruktion durchgeführt, muss das Transplantat gegen diese fixierte hintere Schubladenposition „anarbeiten“. Prädisponierende Faktoren für eine fixierte hintere Schublade sind:

1. männliches Geschlecht

2. vorbestehende Entnahme eines Lig.patellae-Anteils im Rahmen einer Kreuzbandrekonstruktion

3. vorangegangene HKB-Rekonstruktion 4. lange bestehende HKB-Insuffizienz

Findet sich eine fixierte hintere Schublade, wird diese zunächst therapiert (Abb.53). Besteht keine freie Beweglichkeit, ist zuerst eine arthroskopische Arthrolyse angezeigt. Ansonsten empfiehlt sich das Anlegen einer PTS-Schiene. In dieser Schiene wird der Unterschenkel durch einen posterior aufgebrachten tibialen Support im Vergleich zum Oberschenkel nach anterior ge- drückt. Die Schiene wird bei ausgedehnten fixierten Schubladen Tag und Nacht getragen, bei Werten von weniger als $-8 \mathrm{~mm}$ nur nachts. Tagsüber wird dann eine PCL-Orthese (z.B. DonJoy PCL 4Titude Fa. Ormed.djo, Freiburg) angelegt (s. unten).

Der Zustand des Kniegelenks wird nach 6 bis 8 Wochen kontrolliert (erneute gehaltene Röntgenaufnahme). Erst wenn die fixierte hintere Schublade beseitigt ist, erfolgt die stabilisierende Operation.

\section{MR-Tomografie (MRT)}

Bei der frischen Läsion ist die MRT ein wichtiges diagnostisches Hilfsmittel. Mit ihr wird die Rupturart (Komplettruptur, Partialruptur) und der Rupturort (proximal, intermediär, distal) bestimmt (Abb. 54). Nicht selten finden sich ausgedehnte Knochenödeme. Aus deren Lokalisation kann auf den Unfallmechanismus geschlossen werden. So finden sich z.B. nach einem Hyperextensionstrauma Knochenödeme am anterioren Tibiakopfbereich sowie im ventralen Femurbereich.

Bei chronischen Läsionen bietet die MRT keine so entscheidende diagnostische Hilfe. Es ist bekannt, dass das HKB sehr gut vaskularisiert ist und daher selbst unter unzureichender konservativer Therapie manchmal ausheilt, wenn auch unter Elongation. Dies führt dazu, dass häufig bei chronischen Läsionen das HKB vom Radiologen nicht selten als „intakt“ oder „leicht gelockert“ beurteilt wird. Es ist festzustellen, dass die MRT lediglich eine morphologische Darstellung des HKBs bietet, aber keine funktionelle Beurteilung zulässt. Oft ist man dann erstaunt, wie ausgedehnt die posteriore Tibiaverschiebung (hintere Schublade) bei den gehaltenen Röntgenaufnahmen ist. 


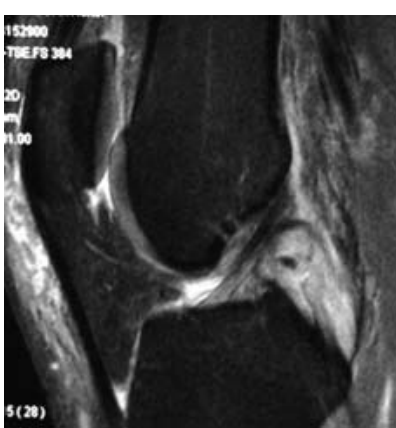

Abb. 54 HKB-Komplettruptur.

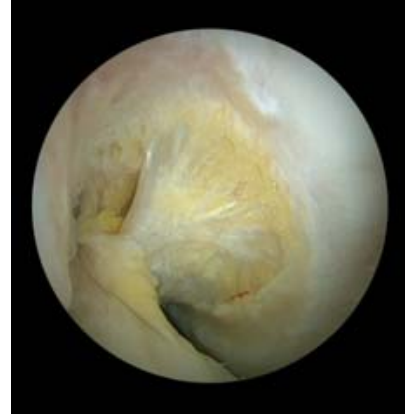

Abb.55 Das HKB erscheint inspektorisch nach Entfernung des Fettkörpers vor dem Ursprungsareal nahezu unauffällig.
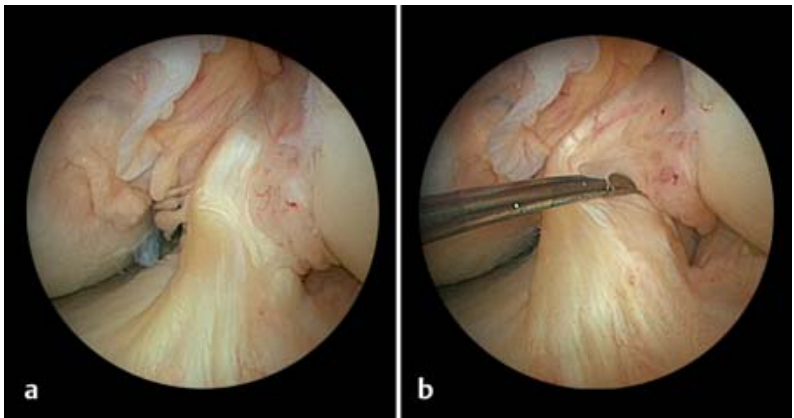

Abb.56a und b Sloppy-ACL-Sign bei chronischer HKB-Insuffizienz. Das VKB liegt komplett „entspannt“ in der Area intercondylaris anterior (a). Die Palpation mit dem Tasthaken zeigt die typische VKB-Elongation (b).
Hinsichtlich des therapeutischen Vorgehens ist es von Bedeutung, ob noch HKB-Strukturen vorhanden sind, oder ob nur ein inhomogenes Narbengewebe vorliegt. Hilfreich ist die MRT zur Beurteilung von intraossären Veränderungen (Ödeme) bedingt durch den chronisch erhöhten Druck im medialen Kompartment und/oder im Femoropatellargelenk [77].

\section{Arthroskopie}

Die Beurteilung der HKB-Läsion durch die Arthroskopie ist bei der frischen Läsion nicht einfach, äußerst schwierig jedoch bei der chronischen Läsion.

\section{Frische Läsion}

Die Indikation zur Arthroskopie ist bei einer frischen HKB-Ruptur nur sehr selten gegeben. Bei der Inspektion von anterior kann der Befund völlig unauffällig sein. Will man das HKB adäquat inspizieren, ist dies nur über einen dorsomedialen Zugang möglich. Da die posteriore Kapsel und/oder mediale oder laterale Strukturen bei einer HKB-Läsion mitverletzt sind, treten bei der Arthroskopie erhebliche Flüssigkeitsmengen durch die rupturierten Kapselanteile in die umgebenden Weichteile aus. Das weitere Vorgehen wird dadurch erschwert. Nicht zuletzt aus diesen Gründen sollte die Indikation zur Arthroskopie sehr zurückhaltend gestellt werden. Keinesfalls sollten die rupturierten HKBBandanteile großzügig entfernt werden.

\section{Chronische Läsion}

Das HKB kann inspektorisch nahezu unauffällig erscheinen (Abb.55). Erst wenn der Fettkörper vor dem HKBUrsprung entfernt wird, können sich Unregelmäßigkeiten zeigen. Wesentlich auffälliger ist dagegen die deutliche
Elongation des VKBs (Sloppy-ACL-Sign). Dies ist unvermeidbar, da sich der Unterschenkel in einer posterioren Position befindet (Abb. 56). Das palpatorisch massiv gelockerte VKB verführt den unerfahrenen Untersucher leicht dazu, den Befund als Elongation oder Insuffizienz des VKB zu beschreiben. In einem derartigen Fall wird leider häufig die Diagnose „VKB-Insuffizienz“ gestellt und von einer gedeckten VKB-Ruptur gesprochen. Das weitere therapeutische Augenmerk richtet sich dann leider auch auf das VKB, welches ersetzt wird. Bei der Analyse unseres Patientenguts (sporthopaedicum Straubing, $\mathrm{n}=2150$ ) mit gesicherten HKB-Rupturen war bei 8\% der Patienten in der Vorgeschichte eine VKB-Rekonstruktion erfolgt. Dadurch kann das Kniegelenk in einer hinteren Schubladenposition fixiert werden, mit der Folge von massiv erhöhten Anpressdrücken im medialen Kompartment und im femoropatellaren Gelenkbereich (Abb. 53). Daher gilt nach wie vor die Grundregel von Müller [52]: Es darf erst dann von einer VKB-Insuffizienz oder VKB-Ruptur gesprochen werden, wenn eine HKB-Läsion ausgeschlossen ist.

Bei der Arthroskopie wird zunächst das „lockere VKB“ palpiert und dann der Unterschenkel nach anterior gezogen. Dann wird das VKB erneut palpiert. Bei intaktem Zustand spannt es sich an. Sollte keine VKB-Anspannung zu verzeichnen sein, können hierfür 2 Gründe verantwortlich sein:

1. Fixierte hintere Schublade. Die Tibia lässt sich nicht in die Neutralposition bewegen. Damit ist eine Anspannung des VKBs nicht möglich, da der Unterschenkel in einer posterioren Position verbleibt.
2. VKB-Insuffizienz. Spannt sich das VKB trotz Erreichen der anterioren Schubladenposition (Neutralposition) nicht an, muss von einer VKB-Insuffizienz ausgegangen werden. In einem derartigen Fall muss auch ein VKBErsatz diskutiert werden.

Bei der Arthroskopie gilt es auch das mediale und laterale Kompartment hinsichtlich degenerativer Veränderungen zu beurteilen. Gleiches gilt für das Femoropatellargelenk. Eigene Untersuchungen haben gezeigt, dass je länger die HKB-Insuffizienz besteht und je ausgedehnter die hintere Schublade ist, desto höhergradige Knorpelläsionen finden sich im medialen Kompartment und im femoropatellaren Gelenkbereich [77, 87].

\section{Staging-Arthroskopie}

Mit der klinischen Untersuchung und gehaltenen Röntgenaufnahmen ist die Diagnose der HKB-Insuffizienz zwar eindeutig zu stellen, schwieriger ist aber die Beurteilung der medialen und lateralen Gelenkecke hinsichtlich Stabilität und Knorpelveränderungen. Daher empfiehlt sich eine Staging-Arthroskopie bei ausgeprägten Instabilitäten zur Beurteilung der medialen und lateralen Stabilität. Findet sich in der 4er-Position eine deutliche Aufklappung des lateralen Gelenkspaltes, muss von einer posterolateralen Instabilität ausgegangen werden. Dies ist oft bei hinteren Schubladenwerten von mehr als - $13 \mathrm{~mm}$ gegeben (gehaltene Röntgenaufnahme). Aber auch bei kleineren Schubladenwerten (z.B. - $9 \mathrm{~mm}$ ) können signifikante posterolaterale Instabilitäten beobachtet werden (Abb. 57). Klinisch sind diese Instabilitäten nicht eindeutig in ihrer Ausdehnung beurteilbar. Daher ist auch bei geringen hinteren Schubladenwer- 


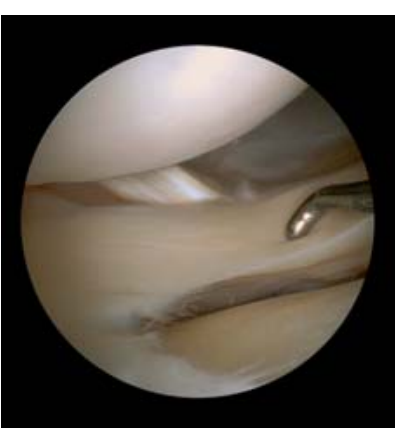

Abb. 57 Staging-Arthroskopie bei chronischer HKB-Insuffizienz. Die Erweiterung des lateralen Gelenkkompartments weist auf die posterolaterale Instabilität hin.

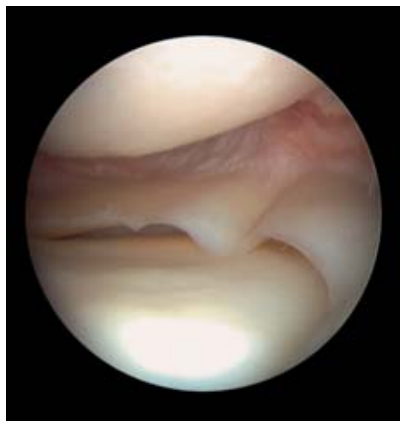

Abb. 58 Staging-Arthroskopie. Der erweiterte mediale Gelenkspalt belegt die mediale Instabilität.

ten im Zweifelsfall eine Staging-Arthroskopie angezeigt. Gleiches gilt für Instabilitäten der medialen Gelenkseite.

Findet sich eine vermehrte Aufklappung des medialen Gelenkspalts, muss von einer Insuffizienz des medialen und dorsomedialen Kapsel-Band-Apparates ausgegangen werden. Der gesamte Innenmeniskus ist in Streckstellung zu überblicken (Abb. 58). In diesem Fall sollten die Hamstringsehnen der ipsilateralen Seite nicht als Transplantat verwendet werden. Besteht eine valgische Beinachse, ist ebenfalls die Entnahme der ipsilateralen ST- und GT-Sehne zu vermeiden. Andernfalls kann eine dekompensierte mediale Instabilität entstehen, die äußerst schwer zu therapieren ist.

\section{Therapie}

In früheren Jahren wurde bei HKB-Läsionen fast ausschließlich die konservative Therapie favorisiert, da das operative Vorgehen mit einer extrem hohen Morbidität für den Patienten verbunden war. Heute kann man aber feststellen, dass bei adäquater operativer Technik die
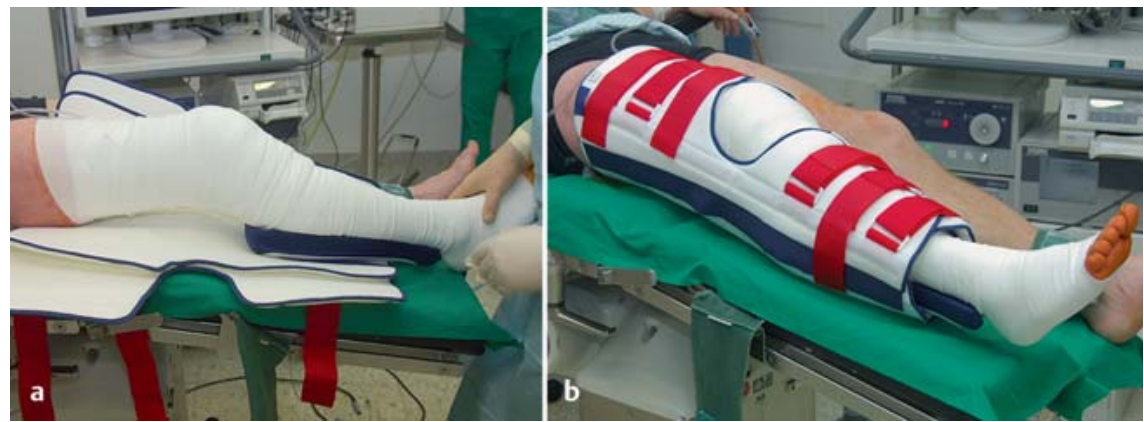

Abb.59a und b PTS-Schiene (postoperative Anwendung). Unter dem Unterschenkel findet sich ein Support (blau), der den Unterschenkel im Vergleich zum Oberschenkel nach anterior drückt (a). Zuerst wird die Schiene femoral geschlossen, danach tibial. Somit wirkt auf den Unterschenkel eine nach anterior gerichtete Kraft, um das HKB zu entlasten (b) (Fa. Medi, Bayreuth).

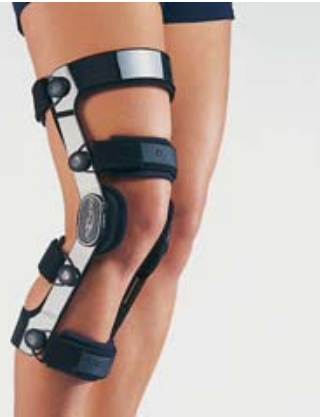

Abb. 60 PCL-Orthese (Donjoy PCL 4Titude, Fa. Ormed.djo, Freiburg).

Morbidität mit der einer VKB-Rekonstruktion identisch ist. Es gilt jedoch zu beachten, dass das präoperative Management wesentlich umfangreicher ist. In Anbetracht der guten Durchblutung des HKBs nimmt die konservative Therapie zudem einen wichtigen Stellenwert ein $[60,85,88]$.

\section{Akute Läsion}

Bei der frischen HKB-Läsion wird zunächst die akute Symptomatik therapiert. Lymphdrainagen dienen der Reduktion des Schwellungszustands. Wie bei der VKB-Therapie (s. oben) gilt es, insbesondere die Faktoren Sportart, Leistungsgrad (Freizeitsportler, Berufssportler), Begleitverletzungen und den Lokalbefund zu berücksichtigen. Darüber hinaus gilt es auch die Rupturart und den Rupturort zu evaluieren. Diese Faktoren werden mit der MRT bestimmt. Findet sich eine komplette Ruptur mit Zerstörung über den gesamten Bandverlauf und gleichzeitig eine signifikante hintere Schublade (gehaltene Röntgenaufnahme $\geq 10 \mathrm{~mm}$ ), sollte beim Hochleistungs- oder Berufssportler ein opera- tives Vorgehen diskutiert werden, wenn dieser beim Sport auf ein stabiles Kniegelenk angewiesen ist. Bei einem konservativen Therapieversuch muss bei Versagen der konsequenten konservativen Therapie ein zusätzlicher Zeitverlust von 3 bis 4 Monaten einkalkuliert werden. Daher wird bei Sportlern mit einer ausgedehnten hinteren Instabilität eher zur frühzeitigen operativen Stabilisierung geraten.

Bei geringen Schubladenwerten $(\leq 10 \mathrm{~mm})$ und geringen Beschwerden wird sofort nach der Verletzung mit einem Aufbautraining des M. quadriceps begonnen. Dieser Muskel ist der wichtigste Agonist des HKB und damit entscheidend für den konservativen Therapieerfolg. Diese Maßnahmen werden durch eine Schienenbehandlung unterstützt. Nachts erhält der Patient eine PTS-Schiene für die ersten 6 bis 8 Wochen (Abb.59). Tagsüber trägt er eine PCL-Orthese (z.B. DonJoy PCL 4Titude, Fa. Ormed.djo, Freiburg) (Abb. 60).

Der Patient kann ab der 3. bis 4 . Woche voll belasten, sofern keine Schmerzen vorhanden sind. In der Nachbehandlung und bei Beginn sportlicher Aktivitäten sind Bergabgehen oder Bergablaufen zu vermeiden, da hierbei extreme Kräfte auf das heilende HKB bzw. das Transplantat einwirken. Wichtig ist der Ausschluss von Begleitverletzungen. Sind der mediale und/oder der laterale Bandapparat verletzt, muss entschieden werden, ob eine primäre operative Stabilisierung dieser Strukturen angezeigt ist (s. unter Komplexverletzung). 


\section{Chronische Läsion}

Liegt die HKB-Läsion länger als 3 Monate zurück, wird von einer chronischen Läsion gesprochen. Die Therapieentscheidung, ob eine konservative oder operative Therapie erfolgt, hängt von der subjektiven Symptomatik und den Begleitverletzungen, aber auch dem Knorpelzustand ab. Klagt der Patient über eine Instabilität, liegt meist eine periphere Instabilität vor. Daher wird wie folgt vorgegangen:

1. Gehaltene Röntgenaufnahmen. Zuerst wird das Ausmaß der posterioren Tibiaverschiebung exakt bestimmt und eine fixierte hintere Schublade ausgeschlossen (s. oben, Abb. 51 und 52). Liegt diese vor, wird zunächst konservativ mit der PTS-Schiene behandelt (Abb.59). Bei Bewegungseinschränkungen erfolgt zuerst eine Arthrolyse und die Weiterbehandlung in der PTS-Schiene.

2. Hintere Schublade $<\mathbf{1 0} \mathbf{m m}$. Vom Schubladenausmaß her kann eine isolierte hintere HKB-Läsion vermutet werden. Es gilt jedoch eine posterolaterale Instabilität auszuschließen, wenn der Patient über ein subjektives Instabilitätsgefühl klagt. Lässt sich durch die Bestimmung der Außenrotation (Dial-Test) keine eindeutige Diagnose stellen, muss eine StagingArthroskopie diskutiert werden.

Weist der Patient keine Instabilität auf, erfolgt zunächst ein intensives Quadrizeps-Training. Gleichzeitig erhält der Patient eine PCL-Orthese (Abb.60). Häufig führen diese konservativen Maßnahmen zur Besserung der subjektiven Beschwerden.

3. Hintere Schublade $>\mathbf{1 2} \mathbf{~ m m}$. Es muss davon ausgegangen werden, dass eine schwerere Läsion vorliegt als nur eine isolierte HKB-Insuffizienz. Daher ist der periphere Kapsel-Band-Apparat sorgfältig zu untersuchen. Im Zweifelsfall wird eine Staging-Arthroskopie empfohlen, um den Knorpel und die peripheren Bandstrukturen zu beurteilen. Findet sich eine ausgeprägte periphere Instabilität, muss dem $\mathrm{Pa}$ tienten bei subjektiver Instabilität neben einer HKB-Rekonstruktion auch eine periphere Stabilisierung empfohlen werden.

Mit Patienten, die keinerlei Beschwerden trotz einer ausgedehnten hinteren Schublade aufweisen, muss differenziert diskutiert werden. Wissenschaftliche Studien belegen, dass bei einer ausgedehnten hinteren Schublade eine erhöhte Inzidenz von Knor- pelschäden im medialen Kompartment und im femoropatellaren Gelenkbereich anzutreffen sind. Diese sind umso ausgeprägter, je länger die Läsion zurückliegt und je ausgedehnter die posteriore Instabilität ist [87]. Andererseits gilt es darauf hinzuweisen, dass ein „Antiarthroseeffekt“ durch die stabilisierende Operation wissenschaftlich bisher nicht belegt werden konnte. Daher muss die Indikation zur Operation gerade beim sportlich aktiven Patienten sorgfältig gestellt werden. Die Operation ist nicht angezeigt, um einen Freizeitsportler wieder seiner sportlichen Aktivität zuzuführen. Vielmehr muss versucht werden, das Gelenk so zu stabilisieren, dass der Patient bei sämtlichen Alltagsaktivitäten beschwerdefrei ist. Anders ist die Situation beim Berufssportler. Hier ist bei subjektiver Instabilität eine stabilisierende Operation anzuraten. Es ist aber mit einem Sportausfall von ca. 1 Jahr zu rechnen. Schon aus diesen Gründen muss bei dieser Patientengruppe versucht werden, bei gravierenden oder bei frischen HKB-Läsionen, insbesondere mit Beteiligung der peripheren Bandstrukturen, eine frühzeitige Stabilisierung anzustreben.

4. Schmerzen und keine Instabilität. Bei einem Patienten, der hauptsächlich über Schmerzen und nicht über eine Instabilität klagt, bestehen nur reduzierte Erfolgsaussichten. Hat die HKB-Insuffizienz das „Schmerzstadium" erreicht, ist die Therapie äußerst schwierig. Meist gelingt es nicht, den Schmerzzustand komplett zu beseitigen. In einem derartigen Fall wird zunächst eine PCL-Orthese verordnet (Abb. 60). Kommt es hierunter zur deutlichen Schmerzreduktion, wird ein operatives Vorgehen empfohlen. Eine konservative Therapie mit Kräftigung des Quadrizeps und Dehnung der Oberschenkelmuskulatur wird ebenfalls fortgesetzt.

5. Abwägung zwischen konservativem und operativen Vorgehen. Besteht Unsicherheit, ob eine Stabilisierung zur Beschwerdebesserung führt, erfolgt ein sog. Brace-Test. Hierbei wird eine PCL-Orthese (z.B. DonJoy PCL 4Titude, Abb. 60) angelegt. Führt dies zur deutlichen Besserung der Symptomatik, ist ein HKB-Ersatz zu empfehlen, auch wenn z. B. nur eine posteriore Instabilität von - $7 \mathrm{~mm}$ vorliegt. Kommt es zu keiner Befundänderung, ist die Indikation zur Operation sorgfältig abzuwägen.
Bei chronischen Instabilitäten ist die Prognose reduziert, wenn schon eine patellofemorale und mediale Arthrose höheren Grades vorliegen. Gleichfalls muss mit reduzierten Ergebnissen gerechnet werden, wenn gleichzeitig eine signifikante mediale Instabilität besteht.

Vor jedem operativen Vorgehen sind folgende Grundregeln zu beachten:

1. Rekonstruktion bei freier oder fast freier Beweglichkeit. Ein Gelenk, das nur bis $90^{\circ}$ beugbar ist, wird zunächst physiotherapeutisch mobilisiert, gegebenenfalls wird eine arthroskopische Arthrolyse vorgenommen. Erst bei einer Beweglichkeit von mindestens $120^{\circ}$ sollte die HKB-Stabilisierung erfolgen.

2. Kombinierte Verletzung mit Beteiligung des HKB und VKB. In diesem Fall darf keinesfalls zuerst das VKB rekonstruiert werden. Dies ist streng kontraindiziert (Abb.51 und 53). Das HKB wird immer zuerst rekonstruiert. Sollte ausreichendes Transplantatmaterial vorhanden sein, kann das VKB in gleicher Sitzung behandelt werden. Liegt gleichzeitig eine mediale Instabilität vor, ist besonders vorsichtig vorzugehen, da das Gelenk sonst in einer zu weit anterioren Position fixiert werden kann.

3. Kombinierte Verletzung von HKB, VKB und peripherer Läsion. Primär wird das HKB und die periphere Läsion therapiert. Ist ausreichend Transplantatmaterial vorhanden, erfolgt auch die VKB-Stabilisierung. Daher ist es von großer Bedeutung, bereits präoperativ zu evaluieren, ob eine periphere Instabilität vorliegt oder nicht.

\section{Operationstechnik (allgemein)}

HKB-Rekonstruktionen erfordern ein differenziertes und professionelles Vorgehen, wenn suffiziente Ergebnisse erzielt werden sollen. Auch beim HKBErsatz müssen der Transplantatauswahl und der Fixationstechnik Beachtung geschenkt werden.

\section{Transplantatauswahl}

Im Prinzip gilt gleiches wie bei der VKBRekonstruktion (s. oben) beschrieben. Das mittlere Drittel der Patellasehne (BTB-Technik) wurde lange Jahre favorisiert. Es zeigten sich jedoch in Anbetracht der vorgegebenen Länge des Ligamentanteils erhebliche Fixationsprobleme. Der spitze Winkel am posterioren Austritt aus dem tibialen Kanal wurde 
für das Versagen einiger Operationen verantwortlich gemacht, sodass eine spezielle tibiale Fixationstechnik (Tibial-Inlay- bzw. Tibial-Onlay-Technik) entwickelt wurde. Studien haben aber gezeigt, dass hierdurch keine biomechanischen Vorteile zu erzielen sind. Operativ ist hierbei immer eine Umlagerung des Patienten in Bauchlage oder eine extreme Seitenlage erforderlich, um den Knochenblock von posterior auf der Tibiarückseite zu fixieren.

Auch aus diesen Gründen hat sich bei der HKB-Rekonstruktion die Verwendung der Hamstringsehnen (ST- und GT-Sehne) zunehmend durchgesetzt. Diese Sehnen können als 4er-Strang oder besser 5er-Strang präpariert werden (Abb.61). Eine Transplantatlänge von $10 \mathrm{~cm}$ wird angestrebt, bei kleineren Patienten (Körpergröße < $160 \mathrm{~cm}$ ) kann ein 8-9 cm langes Transplantat ausreichen. Mit der Entnahme der Hamstringsehnen wird zudem der wichtigste Antagonist des HKB geschwächt. Demgegenüber bleibt das wichtige agonistische System (Patellasehne, Quadrizepssehne) komplett unberührt.

Bei peripheren Instabilitäten wird auch die ST-Sehne der gesunden Gegenseite z.B. für eine posterolaterale Stabilisierung, verwendet. Auch die Quadrizepssehne wird von einigen Autoren als primärer HKB-Ersatz verwendet. Es bleibt jedoch $\mathrm{zu}$ bedenken, dass damit eine Schwächung des gesamten agonistischen Systems verbunden ist. Von uns wird die Quadrizepssehne daher nur als „Revisionstransplantat" verwendet.

\section{Fixationstechnik}

Auch bei der Fixation von HKB-Transplantaten hat sich die Hybridfixation bewährt, um die Vorteile der einzelnen Fixationstechniken zu nutzen bzw. deren Nachteile zu vermeiden (s. oben). Es wird die Fixation mit einem Fixationsbutton in Kombination mit einer unterdimensionierten bioresorbierbaren Interferenzschraube favorisiert. Interferenzschrauben aus Metall sind zu vermeiden, da sie bei einer Revision nur sehr mühsam zu entfernen sind. Dies gilt insbesondere für sehr weit in den tibialen Kanal eingedrehte Schrauben (Abb.62).

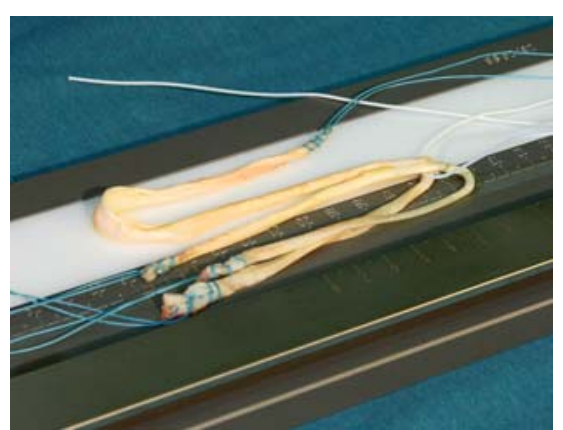

Abb. 61 Präparation des HKB-Transplantats als 5-fach-Strang aus ST- und GT-Sehne.

\section{Platzierung der Bohrkanäle}

Auch beim HKB-Ersatz kommt der Platzierung der Bohrkanäle entscheidende Bedeutung zu. Früher suchte man den isometrischen HKB-Punkt. Dieser liegt nach experimentellen Studien zwischen dem anterolateralen und posteromedialen Bündel $[64,65]$. Daher ist eine isometrische Rekonstruktion des HKB nicht sinnvoll. Vielmehr sollte der anatomische Ersatz der HKB-Struktur angestrebt werden.

Wie auch das VKB, so besteht auch das HKB aus 2 funktionellen Bündeln. Es wird ein sehr kräftiges anterolaterales $(\mathrm{AL})$ von einem weniger kräftigen posteromedialen (PM) Bündel unterschieden. Im Vergleich zum HKB-Querschnitt (100\%) sind die Insertionsareale dreimal so groß (300\%). Daher sollte keinesfalls die komplette HKB-Struktur reseziert werden. Von uns wird die isolierte Rekonstruktion des AL-Bündels bevorzugt, wobei gleichzeitig das PM-Bündel belassen wird. Dieses Vorgehen ist zwar arthroskopisch sehr anspruchsvoll, die belassenen HKB-Strukturen umscheiden und schützen aber das eingebrachte Transplantat. Ansonsten sieht selbst ein Transplantat mit einem Durchmesser von 10 oder $11 \mathrm{~mm}$ nach Entfernung der gesamten HKB-Struktur sehr „einsam“ und ungeschützt aus.

\section{Femoraler Kanal}

Der Bohrkanal für das AL-Bündel wird sehr weit anterior und oben in der Fossa intercondylaris angelegt (12.30-Uhrbzw. 11.30-Uhr-Position). Der Zentrumspunkt liegt etwa $5 \mathrm{~mm}$ von der KnorpelKnochen-Grenze entfernt (Abb. 63).

\section{Tibialer Kanal}

Der tibiale Kanal wird etwas lateral des Zentrums der tibialen Insertion lokalisiert. Manchmal muss bei unzureichender Transplantatlänge hier ein gewisser
Kompromiss eingegangen werden. Der Zentrumspunkt sollte ca. $1,5 \mathrm{~cm}$ distal des Tibiaplateaus liegen.

\section{Spezielle Operationstechnik}

Zum HKB-Ersatz werden sowohl die STals auch die GT-Sehne benötigt. Findet sich kein Genu valgus und keine ausgeprägte mediale Instabilität, werden die beiden Sehnen von der ipsilateralen Gelenkseite entnommen. Findet sich eine mediale Instabilität oder eine periphere, z.B. posterolaterale Stabilisierung, sollte auf die gesunde Gegenseite ausgewichen werden. Beide Sehnen werden von Muskelgewebe und instabilen Sehnenanteilen befreit und auf dem Sehnenboard präpariert. Beträgt die Länge der ST-Sehne $30 \mathrm{~cm}$, wird versucht, ein $10 \mathrm{~cm}$ langes 5-Strang-Transplantat zu präparieren. Die Sehnen werden mit Fäden armiert. Die ST-Sehne trägt zu diesem Transplantat einen 3er-Strang, die GTSehne einen 2er-Strang bei (Abb.61). Nach Bestimmung des Transplantatdurchmessers werden die Bohrkanäle platziert.

Der Arthroskopzugang erfolgt hoch anterolateral, um noch einen tieferen lateralen Zugang platzieren zu können, der für die Platzierung des femoralen Bohrkanals erforderlich ist. Der mediale Instrumentenzugang erfolgt ebenfalls hoch medial. Nach Entfernung des Fettkörpers vor dem HKB-Ursprung erfolgt die Platzierung des tiefen anterolateralen Zugangs zum Zentrumsbereich des femoralen Bohrkanals, der zuvor mit dem Zielgerät markiert wurde (Abb. 63). Es erfolgt das Vorbohren eines Drahts, der anschließend mit einem 4,5-mmBohrer überbohrt wird (Abb. 64). Dann wird die Länge des femoralen Kanals bestimmt. Da dieser oft wesentlich kürzer ist als beim VKB-Ersatz, muss sorgfältig vorgegangen werden, wenn der Bohrkanal auf den endgültigen Durchmesser erweitert wird. Die Perforation der medialen Femurkortikalis ist unbedingt zu vermeiden. Anschließend wird das ALBündel vorsichtig mit dem Shaver und dem HF-Messer entfernt (Abb. 65).

Dann wird unter arthroskopischer Kontrolle ein dorsomedialer Zugang platziert. Mit dem Shaver wird der distale Anteil der dorsalen Kapsel, die direkt über den tibialen HKB-Ansatz verläuft, abgelöst, um die HKB-Insertion darzustellen. Ein Schlitz wird präpariert, damit eine Verbindung zwischen anteriorem und posterioren Gelenkraum ent- 


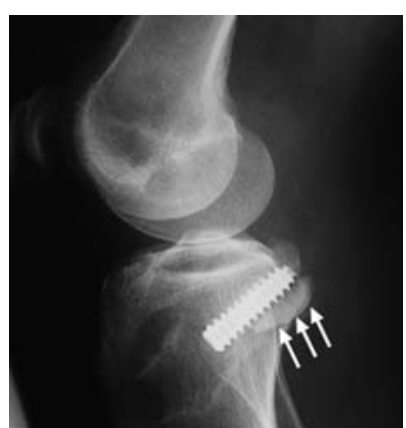

Abb. 62 Weit nach posterior eingedrehte Metallinterferenzschraube. Vor einer erneuten HKB-Rekonstruktion - es besteht eine ausgeprägte posteriore Instabilität - muss die Schraube entfernt werden. Die Revision gestaltet sich durch den nach kaudal ausgebrochenen Knochenteil (Pfeile) noch schwieriger.
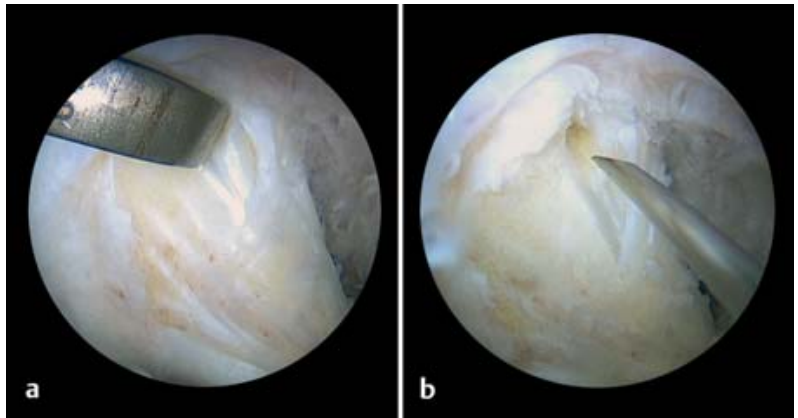

Abb.63a und b Platzierung des femoralen Bohrkanals. Bestimmung des Zentrumspunkts mit dem Zielgerät (a) und Auslotung des tiefen anterolateralen Zugangs mit der Zielkanüle (b).

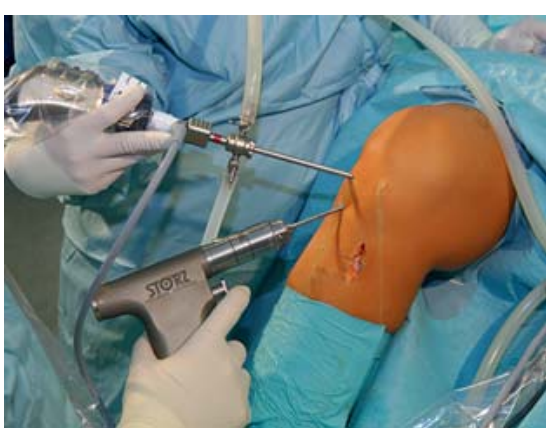

Abb. 64 Anlage des femoralen Bohrkanals über den tiefen anterolateralen Zugang.

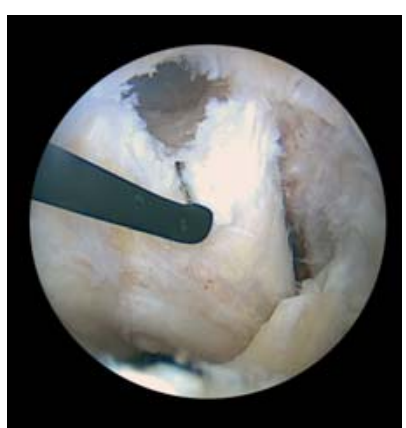

Abb. 65 Partielle Entfernung des AL-Bündels mit dem HF-Messer.

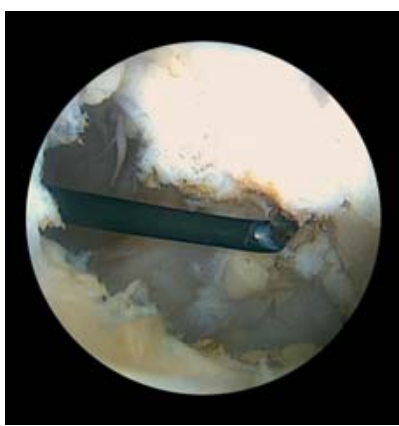

Abb. 66 Präparation der Verbindung zwischen anteriorem und posteriorem Gelenkbereich lateral des HKB-Rests.

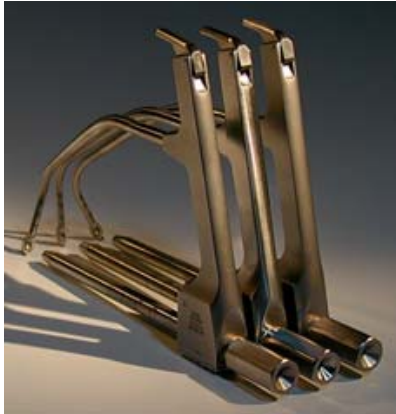

Abb. 67 Tibiale Zielgeräte (nach rechts und links gebogen sowie gerade) (Fa. Karl Storz, Tuttlingen).

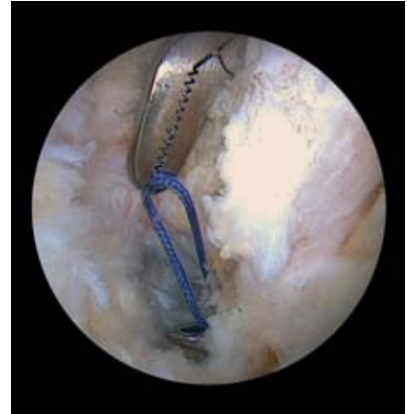

Abb. 68 Fassen des tibialen Durchzugsfadens mit einer Fasszange (Sicht über dorsomedialen Zugang).

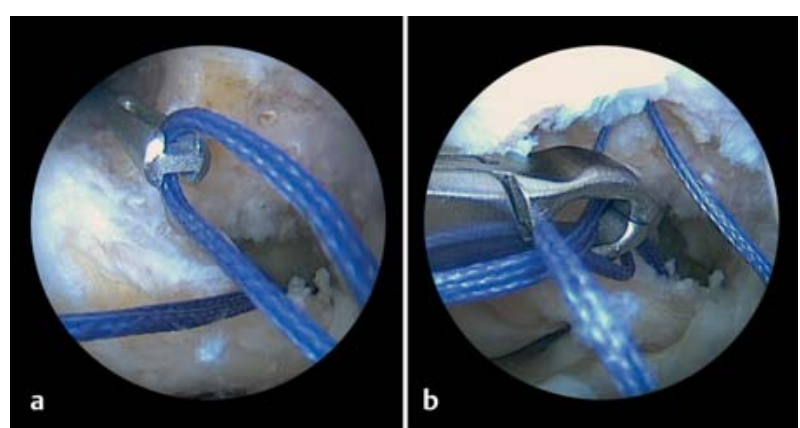

Abb. 69a und b Einführen des Bohrdrahts mit Fadenschlaufe in den femoralen Kanal (a). Durch diese Schlaufe wird der tibiale Durchzugsfaden mit einer speziellen Fadenfasszange gefasst und durch den medialen Instrumentenzugang herausgezogen (b). steht (Abb. 66). Da große Teile des HKBs belassen werden, ist dieser Operationsschritt nicht einfach. Es werden gebogene tibiale Zielgeräte verwendet, um die HKB-Insertion adäquat zu erreichen (Abb. 67). Nach Einführen des tibialen Zielgeräts wird über den dorsomedialen Zugang ein Wechselstab eingeführt und die Optik nach posterior umgesteckt. Liegt das Zielgerät im Zentrum der HKBInsertion, lässt sich die Länge des tibialen Bohrkanals am Zielgerät ablesen. Der Bohrdraht wird dann so in der Bohrmaschine eingespannt, dass er den
Zielbereich des HKB-Zielgeräts nicht nach posterior passieren kann. Unter arthroskopischer Kontrolle wird der K-Draht vorgebohrt. Anschließend wird der tibiale Kanal auf den endgültigen Transplantatdurchmesser sequenziell in 2-mm-Schritten erweitert.

Durch den tibialen Kanal wird eine Ahle mit eingelegtem Faden eingeführt, der mit einer Fasszange gefasst und über den medialen Instrumentenzugang herausgezogen wird (Abb. 68). Damit ist der tibiale Durchzugsfaden platziert.
Die Optik wird nach anterolateral zurückgesteckt und ein Bohrdraht mit eingelegter Fadenschlaufe über den tiefen anterolateralen Zugang in den femoralen Kanal eingeführt. Durch die Fadenschlaufe wird der tibiale Durchzugsfaden mit der Fadenfasszange gefasst und über den medialen Instrumentenzugang ausgezogen (Abb.69). Dann wird am femoralen Faden gezogen, um den tibialen Durchzugsfaden durch den femoralen Kanal zu ziehen. 
Nachdem das Transplantat in Abhängigkeit der femoralen Kanallänge vorbereitet ist - meist wird es $20 \mathrm{~mm}$ in den femoralen Kanal eingezogen -, erfolgt der Einzug (Abb. 70). Hilfreich ist ein spezielles HKB-Elevatorium, das über den dorsomedialen Zugang eingeführt wird, den Durchzugsfaden unterfährt und kontrolliert nach posterior hebelt. Hiermit wird das Umlenken des Transplantats am Ausgang des tibialen Bohrkanals erheblich erleichtert (Abb. 70b). Unter konstantem Zug wird das Transplantat eingezogen, bis die Flippmarkierung den Eingang des femoralen Bohrkanals erreicht. Dann wird der Fixationsbutton geflippt und das Transplantat zurückgezogen, die Flippmarkierung erscheint wieder intraartikulär. Anterior des Transplantats findet sich ein Spalt, in den eine bioresorbierbare Interferenzschraube mit einer Größe von $6 \times 19$ oder $7 \times 19$ eingedreht wird. Zunächst wird das Transplantat unter Spannung gehalten. Hat die Schraube gefasst, wird die Spannung reduziert und die Schraube dreht sich nach unten. Das Transplantat liegt danach anterior der Schraube im Bohrkanal und weist eine Konfiguration auf, die weitgehend dem nativen HKB entspricht (Abb. 71). Unter Zug spannt sich das VKB an (Abb. 71 a). Nach Umstecken der Optik in den dorsomedialen Zugang lässt sich der Transplantatverlauf auch von posterior darstellen (Abb. 71 b).

Vor der tibialen Fixation erfolgt die möglicherweise gleichzeitig erforderliche periphere Rekonstruktion. Insbesondere posterolaterale Instabilitäten sind häufig anzutreffen und müssen entsprechend therapiert werden, um eine Überlastung des HKB-Transplantats
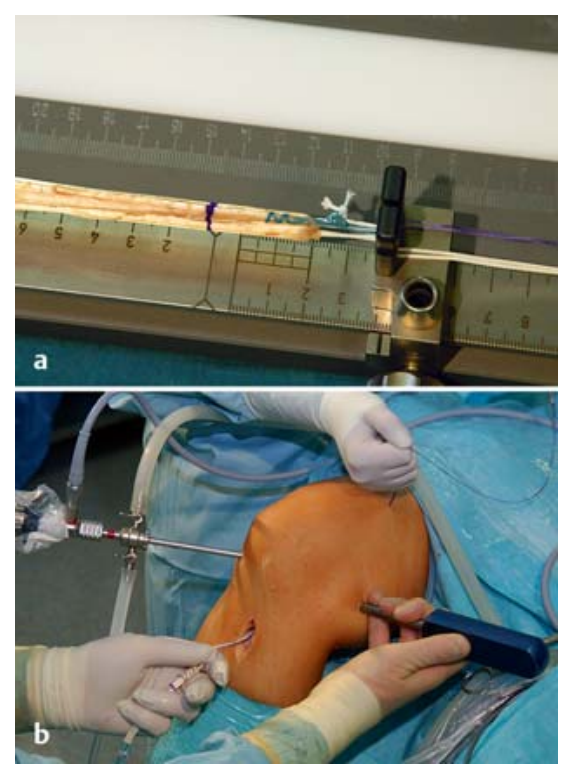

Abb. 70 a und b Transplantateinzug. Fertig vorbreitetes Transplantat auf dem Sehnenboard (a). Der Einzug wird durch ein spezielles HKB-Elevatorium, das über den dorsomedialen Zugang eingeführt wurde, erleichtert (b).

zu verhindern. In diesem Fall erfolgt zunächst die posterolaterale Stabilisierung mit einer modifizierten Larson-Technik [47]. Auf die operativen Details soll an dieser Stelle nicht eingegangen werden.

Erst nach Fixation der posterolateralen Stabilisierung erfolgt die tibiale Fixation des HKB-Transplantats in $60^{\circ}$ Beugung und unter gleichzeitigem anterioren Schubladenstress. Die bioresorbierbare Interferenzschraube wird nach Dilatation des Spalts zwischen Transplantat und Bohrkanalwand in der 3.00-UhrPosition gestartet. Es wird eine perforierte bioresorbierbare Interferenz- schraube (z.B. Megafix-P, Größe $8 \times$ 28 oder $9 \times 28$ bzw. $9 \times 35$ ) verwendet. Alternativ können auch CompositeSchrauben gleicher Größe benutzt werden.

Während des Eindrehens wandert die Schraube entsprechend der Eindrehrichtung in die 6.00-Uhr-Position, sodass sie abschließend unterhalb, d.h. kaudal des Transplantats liegt (Abb. 72). Die Eindrehtiefe wird mithilfe der Tiefenmarkierungen auf dem Schraubendreher exakt kontrolliert. Da nicht mit einer überdimensionierten Interferenzschraube fixiert wird - diese könnte auch zur Läsion des Transplantats führen -, erfolgt die tibiale Fixation ebenfalls als Hybridfixation. Nach der Schraubenfixation wird mit dem Setzgerät ein Bett wie beim VKB-Ersatz (Abb. 45) präpariert, der Endotack eingesetzt und die Armierungsfäden der Sehnenenden geknotet.

Nach dem Einlegen von Redon-Drainagen und dem Wundverschluss (Abb. 73) wird das Kniegelenk in einer PTS-Schiene gelegt (Abb. 59).

Für die detaillierte Darstellung der einzelnen Operationsschritte wird auf die Operationslehren verwiesen $[83,88]$.

\section{Nachbehandlung}

Das Bein wird für 6 Wochen Tag und Nacht in der PTS-Schiene gelagert. Aus der Schiene heraus sind passiv geführte Bewegungen aus Bauchlage, in den ersten beiden Wochen bis $20^{\circ}$, in der 3 . und 4 . Woche bis $40^{\circ}$ und in der 5 . und 6 . Woche bis $60^{\circ}$ erlaubt. Eine aktive Beugung gegen Widerstand ist in den
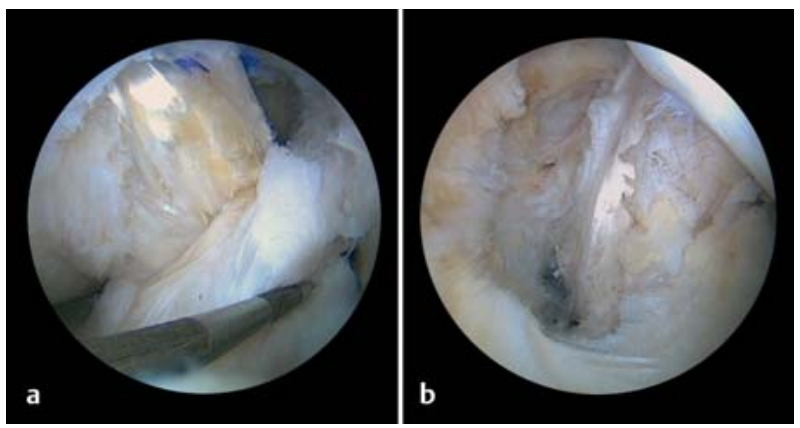

Abb. 71 HKB-Transplantat nach femoraler Fixation. Unter Zug an den Fixationsfäden des Transplantats zeigt sich eine komplette Anspannung des VKB (a). Das Transplantat wird von den erhaltenen HKB-Strukturen eingescheidet, wie die abschließende Inspektion über den dorsomedialen Zugang zeigt (b).

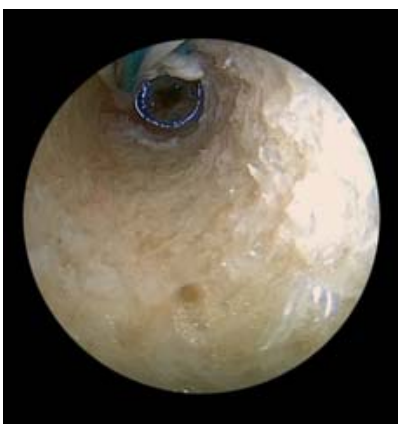

Abb. 72 Lagekontrolle der tibialen Interferenzschraube. Die Optik wird hierzu von anterior in den tibialen Kanal eingeführt. Die Schraube liegt in der 6-UhrPosition.

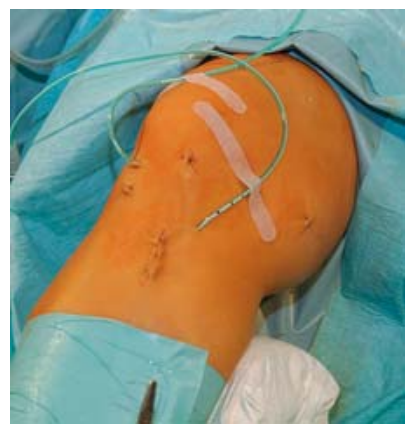

Abb. 73 Endzustand nach HKBRekonstruktion. Anhand der Narben kann nur der Erfahrene erkennen, dass eine HKB-Rekonstruktion erfolgt ist. 
ersten 12 Wochen unbedingt $\mathrm{zu}$ vermeiden. Gleichfalls beginnt der Patient bereits in den ersten postoperativen Tagen mit aktiven Quadrizepsübungen, um den wichtigsten HKB-Agonisten zu stärken.

Lymphdrainagen reduzieren den Schwellungszustand. Eine Elektrotherapie dient der zusätzlichen Kräftigung des M. vastus medialis. In den ersten beiden Wochen ist Bodenkontakt bis $10 \mathrm{~kg}$ erlaubt, in der 3. und 4. postoperativen Woche wird die Belastung auf $20 \mathrm{~kg}$ und in der 5 . und 6 . Woche auf halbes Körpergewicht gesteigert. Ab der 7. Woche erfolgt der Übergang zur Vollbelastung. Die PTS-Schiene wird danach nur noch nachts getragen, ab der 6 . postoperativen Woche wird tagsüber eine PCL-Orthese (z.B. DonJoy 4Titude PCL, Abb. 60) angelegt.

Gerade bei komplexen Rekonstruktionen mit HKB-Rekonstruktion und Stabilisierung der posterolateralen Gelenkecke ist eine stationäre Nachbehandlung ab der 10. postoperativen Woche angezeigt. Insbesondere bei Spitzensportlern und Berufssportlern ist eine optimierte Nachbehandlung in engem Kontakt zwischen Physiotherapeut und Operateur sowie dem Vereinsarzt erforderlich.

\section{Periphere Bandverletzungen (medialer - dorsomedialer Band- komplex, lateraler - posterolateraler Bandkomplex)}

Ursache

Valgustraumen mit Rotationskomponenten führen zur Läsion der medialen Gelenkecke. Die Läsionen reichen von einer Partialruptur des medialen Seitenbands bis hin zur komplexen Zerreißung des medialen und dorsomedialen Kapsel-Band-Apparates (hinteres Schrägband). Letzteres ist wesentlich für die mediale Stabilisierung in Streckstellung verantwortlich [31,52].

Bei Varustraumen ist die laterale Gelenkseite betroffen. Auch hier reicht das Verletzungsspektrum von Partialrupturen bis hin zur komplexen Zerreißung des gesamten lateralen und posterolateralen Bandkomplexes einschließlich des Tractus iliotibialis, M. biceps femoris und M. popliteus.

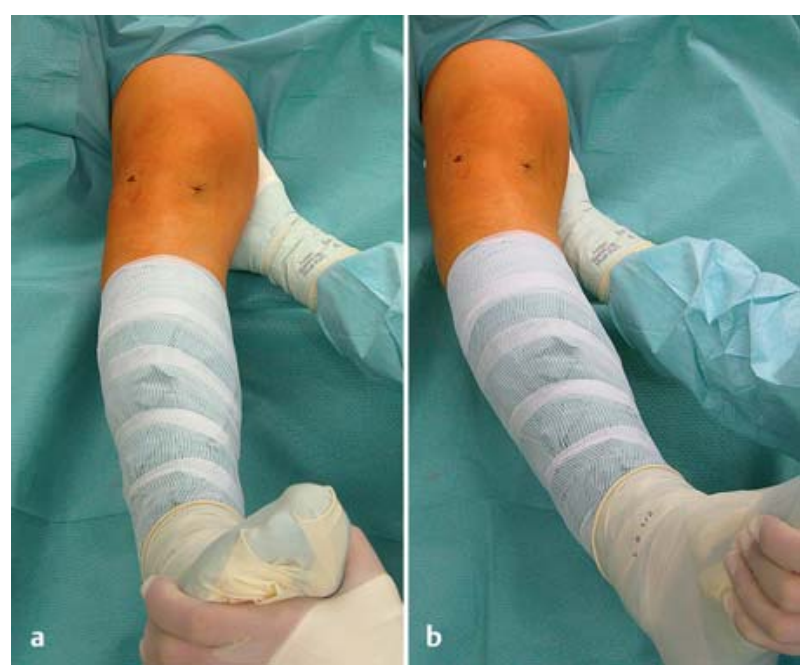

Abb. 74 a und b Komplette Ruptur der lateralen und posterolateralen Strukturen. Ausgangsbefund (a). Unter Varusstress zeigt sich die massive Aufklappung und deutliche Überstreckung (b).

\section{Symptomatik}

\section{Akute Läsion}

Bei Partialrupturen stehen häufig massive Schmerzen und Bewegungseinschränkung im Vordergrund. Vielfach lässt sich der Untersucher allzu sehr vom Schmerzbild beim diagnostischen Vorgehen beeinflussen. Werden starke Schmerzen angegeben, wird häufig von einer schwerwiegenden Verletzung ausgegangen. Beklagen die Patienten jedoch keinerlei oder nur minimale Schmerzen, so wird die Diagnostik manchmal sogar oberflächlich gehalten. Hierzu ist festzustellen, dass insbesondere das schmerzfreie oder schmerzarme Gelenk häufig sehr gravierend verletzt ist. Sind Kapsel-Band-Strukturen komplett zerrissen, sind auch die feinen Nervenfasern, die in den Bändern verlaufen, durchtrennt. Es erfolgt keine Weiterleitung der „Schmerzinformation“. Bei einer Partialruptur entstehen dagegen Dehnungen der feinen Nerven und Hämatome im Bandverlauf können Druck auf die Nervenfasern ausüben. Daher wird bei derartigen Verletzungsmustern häufig über sehr starke Schmerzen geklagt. Demnach sind $\mathrm{Pa}-$ tienten, die nur über geringe Schmerzen klagen, besonders sorgfältig zu untersuchen, oft finden sich schwerwiegende komplexe Bandverletzungen. Ein sehr starkes Schmerzbild muss dagegen noch an intakte Strukturen denken lassen, da noch eine ausreichende „Schmerzweiterleitung“ gegeben ist. Besonders häufig ist die Teilruptur des medialen Seitenbands (s. Kap. Meniskus).
Chronische Läsion

Chronische Instabilitäten des medialen bzw. lateralen Bandapparates sind oft zusammen mit Kreuzbandläsionen anzutreffen. Seltener liegen sie isoliert vor. Insbesondere bei Sportarten mit schnellen Richtungswechseln können derartige Läsionen symptomatisch werden. Die Therapie ist dann problematisch, wenn ungünstige Beinachsen (mediale Instabilität bei valgischer Beinachse bzw. laterale Instabilität bei varischer Achse) und gleichzeitig eine sportliche Aktivität mit Richtungswechseln vorliegt.

\section{Klinische Diagnostik}

\section{Akute Läsion}

Ist die mediale bzw. laterale Aufklappbarkeit sehr schmerzhaft, muss von einer Teilruptur ausgegangen werden. Die betroffene Gelenkseite wird sorgfältig palpiert. Insbesondere im Bereich des medialen Seitenbands lässt sich der Rupturverlauf schon lokalisieren. Gravierende Verletzungen liegen meist dann vor, wenn das Kniegelenk überstreckbar und deutlich nach medial bzw. lateral aufklappbar ist. Eine deutliche Aufklappbarkeit in Hyperextension spricht für eine gravierende Läsion der betroffenen Gelenkseite, oft mit Beteiligung des HKB (Abb. 74).

\section{Chronische Läsion}

Chronische Instabilitäten des medialen und/oder lateralen Kapsel-Band-Apparates müssen meist dann therapeutisch angegangen werden, wenn sie zu einer Instabilität bei gestrecktem Gelenk füh- 
ren und/oder gleichzeitig eine Kreuzbandläsion vorliegt. Leichte Instabilitäten, die zu keinerlei Symptomatik führen, jedoch klinisch nachweisbar sind, sollten in 6-monatigen Abständen kontrolliert werden, um eine Dekompensation der Instabilität rechtzeitig zu erkennen.

\section{Bildgebende Diagnostik}

\section{Röntgen}

Standardaufnahmen in 2 Ebenen zum Ausschluss von knöchernen Verletzungen sind obligat. In Abhängigkeit von evtl. vorliegenden Begleitverletzungen (v.a. auf HKB-Läsion) sind weitere Spezialaufnahmen angezeigt (s. oben).

\section{MR-Tomografie}

Hiermit lässt sich die Lokalisation der Ruptur meist eindeutig bestimmen. Insbesondere muss die komplette distale Ruptur des Innenbands ausgeschlossen werden. In diesem Fall kann das Innenband über dem Pes anserinus schlagen. In diesen seltenen Fällen ist eine konservative Therapie nicht möglich, da das Innenband nicht an seiner normalen Insertion anheilen kann. Die operative Intervention ist hier unbedingt angezeigt.

\section{Arthroskopie}

\section{Akute Läsion}

Bei akuten Komplexläsionen ist eine Arthroskopie kaum indiziert, es sei denn, es besteht der Verdacht einer intraartikulären Blockade. Vielmehr besteht die Gefahr, dass Spülflüssigkeit aus der rupturierten Kapsel austritt und in das Subkutangewebe verläuft. Wird in gleicher Sitzung eine Rekonstruktion bzw. Refixation der Bandrupturen angestrebt, kann sich das Vorgehen hierdurch äußerst schwierig gestalten. Ist eine Arthroskopie trotzdem angezeigt, sollte sie von einem sehr erfahrenen Arthroskopiker unter geringem Flüssigkeitsdruck und in möglichst kurzer Zeit durchgeführt werden.

\section{Chronische Läsion}

Bei chronischen Instabilitäten ist es nicht immer einfach, eine mediale von einer lateralen Instabilität zu differenzieren. Wenn der Eindruck besteht, das Gelenk ist sowohl nach medial als auch nach lateral instabil, sollte eine StagingArthroskopie erwogen werden (s. oben, Abb. 57 und 58). Hierbei kann die Insta- bilität eindeutig verifiziert werden. Gleichfalls gilt es die Läsionen der Kreuzbänder und Knorpelschäden zu beurteilen. In gleicher Sitzung kann gegebenenfalls auch die definitive Therapie erfolgen.

\section{Therapie}

\section{Akute Läsion}

Teilrupturen oder isolierte Rupturen des medialen Seitenbands, bei denen die komplette distale Ruptur ausgeschlossen ist, werden konservativ therapiert. Es ist hierbei aber immer wieder erstaunlich, wie lange der Schmerzzustand gerade bei Teilrupturen persistieren kann.

Die konservative Therapie erfolgt mit einer $20^{\circ}$-Mekronschiene für 3 Wochen Tag und Nacht, danach kann tagsüber auf eine Orthese übergegangen werden. Nachts sollte die $20^{\circ}$-Schiene weiter getragen werden. Gleichzeitig sind passive und aktive Bewegungsübungen möglich.

Ein länger persistierender Schmerzzustand im Bereich des Skipunkts (medialer Femurepikondylus) kann nach 6 Wochen mit einer Injektion eines Lokalanästhetikums unter Bemischung eines Kortikoids therapiert werden (Cave: Dopingproblematik beim Sportler).

Findet sich bei der klinischen Untersuchung eine deutliche mediale oder laterale Aufklappung in Streckstellung oder sogar in Überstreckung, muss mit einer gravierenden Ruptur der posteromedialen bzw. posterolateralen Kapselschale gerechnet werden. Derartige Läsionen heilen unter konservativen Bedingungen nur unzureichend aus. Zudem sind häufig noch andere Bandstrukturen, z. B. HKB und/oder VKB verletzt. Bei derart gravierenden peripheren Rupturen ist die primäre Refixation zu empfehlen.

\section{Mediale Gelenkseite}

In früheren Jahren erfolgte die Naht bzw. Refixation der rupturierten Bandanteile oft mit einer Krallenplatte oder einem oder mehrerer Staples. Auch die Naht der rupturierten Bandanteile über eine Schraube mit Unterlegscheibe ist hinlänglich bekannt. Ein großer Nachteil dieser Refixationstechniken besteht darin, dass die rupturierten Bandstrukturen hierbei auf einem Punkt fixiert wer- den. Dies ist weder biomechanisch noch anatomisch sinnvoll. Nach derartigen Refixationen sind die Kniegelenke entweder in ihrer Beweglichkeit massiv eingeschränkt oder massiv ausgelockert. Heute ist unbedingt eine anatomische Refixation anzustreben. Hier bieten sich kleine bioresorbierbare Ankersysteme, wie sie auch in der Schulterchirurgie verwendet werden, an. Diese Anker werden an den anatomischen Insertionsorten platziert und die rupturierten Bandstrukturen anatomisch exakt fixiert.

\section{Laterale Gelenkseite}

Tierexperimentelle Untersuchungen haben gezeigt, dass komplexe Läsionen der lateralen Gelenkseite unter konservativer Therapie nicht ausheilen. Persistierende laterale und posterolaterale Instabilitäten bereiten auch heute noch große therapeutische Probleme. Daher sollten komplexe laterale und posterolaterale Läsionen akut angegangen werden. Es ist hierbei immer wieder erstaunlich, wie ausgedehnt derartige Rupturen sein können. So können der Tractus iliotibialis wie auch der M. biceps femoris komplett von ihrer Insertion abgerissen sein. Häufig sind auch der M. popliteus und das laterale Seitenband mitbetroffen. Auch bei der lateralen Refixation bietet sich die Verwendung von bioresorbierbaren Ankern an, um die rupturierten Bandanteile im Bereich ihrer normalen anatomischen Insertion zu refixieren (Abb. 75). Die Refixation mit Staples oder Schrauben sollte verlassen werden, da diese Implantate oft auftragen und bei Wiedereintritt der Beweglichkeit das Gleiten der einzelnen Gewebeschichten gegeneinander behindern können. Zudem müssen derartige Implantate oft vor einer evtl. erforderlichen späteren Revision wieder entfernt werden.

Auf die Refixation der Popliteussehne und des lateralen Seitenbands, aber auch der dorsolateralen Kapsel ist besonders zu achten. Bei ausgedehnten lateralen Instabilitäten kann es hilfreich sein, die laterale Refixation mittels einer PDS-Kordel, die durch das Fibulaköpfchen gezogen wird und im Femur etwas anterior superior des Ursprungs des lateralen Seitenbands in einem zuvor angelegten femoralen Tunnel verläuft, zu schützen. Sollte eine Instabilität persistieren, kann in einer zweiten Sitzung ein autologes Sehnentransplantat verwendet werden. Alternativ besteht natürlich auch die Möglichkeit, hierfür pri- 
mär auch die ipsilaterale ST-Sehne zu verwenden. Insbesondere wenn gleichzeitig Kreuzbandverletzungen vorliegen, sollte jedoch nicht unnötig Sehnenmaterial „verschwendet“ werden.

Die Therapie derartiger komplexer Verletzungen mit Beteiligung des VKB und/ oder HKB sollte dem Spezialisten vorbehalten bleiben. Nach einer insuffizienten Primärversorgung können persistierende laterale und mediale Instabilitäten auftreten, die im Nachhinein nur äußerst schwierig zu behandeln sind. Auf die detaillierte Darstellung der Therapie der chronischen medialen und lateralen Instabilitäten wird an dieser Stelle verzichtet, es wird auf die speziellen Operationslehren verwiesen.

\section{Knorpel}

Knorpelläsionen sind häufig zu finden. Sie können traumatischer, aber auch degenerativer Genese sein. Im Folgenden wird hauptsächlich auf die traumatischen Knorpelläsionen eingegangen. Der arkadenförmig aufgebaute hyaline Gelenkknorpel ist prädestiniert, den auftretenden Druck bei Belastungen adäquat aufzunehmen und zu verteilen. Bei Knorpelschäden ist dieser Abfederungsmechanismus gestört und der Druck wird direkt auf den Knochen übertragen. Es ist wichtig festzustellen, dass der Knorpel keine Nervenfasern aufweist. Ansonsten würden auch beim Gesunden Schmerzen auftreten, wenn er z.B. mehrere Treppenstufen herunterspringt oder die Treppe hinabsteigt.

\section{Ursache}

Eine vermehrte axiale Kompression, aber auch repetitive Traumen können Ursache von Knorpelläsionen sein. Gleichfalls können sie bei Kapsel-BandVerletzungen auftreten. Auch nach Patellaluxationen finden sich Knorpelläsionen im Bereich der medialen $\mathrm{Pa}-$ tellafacette und der lateralen Femurkondylenwange.

\section{Symptomatik}

Bei osteochondralen Läsionen ist ein Hämarthros anzutreffen. Bei isolierten Knorpelverletzungen ist dies eher selten. Die Symptomatik kann von nahezu Beschwerdefreiheit bis zu massiven Schmerzen reichen. Sind größere Knorpelfragmente ausgebrochen, können Gelenkblockaden auftreten. Typische Symptome sind nicht bekannt.

\section{Diagnostik}

Ein typisches diagnostisches Zeichen für Knorpelläsionen ist nicht bekannt. Lokale Druckschmerzen oder unklare Schmerzen im Gelenk, manchmal mit einem Krepitieren verbunden, können auf Knorpelläsionen hinweisen, müssen es aber nicht. Durch die alleinige klinische Diagnostik lässt sich eine Knorpelläsion weder bestätigen noch widerlegen.

Es gilt aber immer, den Kapsel-Band-Apparat und das Femoropatellargelenk zu untersuchen. Insbesondere ein positiver Apprehension-Test mit druckschmerzhaftem medialen Retinakulum kann auf eine Patellaluxation oder Patellasubluxation hinweisen.

\section{Bildgebende Diagnostik}

Röntgen

Radiologisch finden sich nur dann Hinweise auf Knorpelläsionen, wenn fortgeschrittene degenerative Veränderungen mit Gelenkspaltverschmälerung und Osteophyten vorliegen. Ansonsten sind die Röntgenbilder meist unauffällig, sofern es sich nicht um den Ausbruch eines osteochondralen Fragments handelt. In diesem Fall ist eine kleine ossäre Schuppe zu erkennen.

\section{MR-Tomografie}

Die MRT ist bei Knorpelläsionen sehr hilfreich. Nicht nur die Ausdehnung der Knorpelläsion, sondern auch ossäre Begleitödeme (Bone Bruises) sind zu diagnostizieren. Diese sind meist größer als vermutet (Abb. 76). Gleichfalls können Begleitläsionen am Meniskus bzw. am Kapsel-Band-Apparat evaluiert werden.

\section{Arthroskopie}

Arthroskopisch lässt sich eine Knorpelläsion eindeutig hinsichtlich Ausdehnung und Ort verifizieren. Gleichfalls sind Begleitverletzungen zu evaluieren.

Bei der Arthroskopie sollte jedoch nicht nur die Diagnostik, sondern auch die Therapie erfolgen. Ein abgelöstes Knorpelstück, dass nicht refixierbar ist, wird entfernt. Der entstandene Defekt wird in Abhängigkeit der Größe behandelt (s. unten).

\section{Therapie}

Die Therapie der Knorpelläsion hängt im Wesentlichen von der Art, der Lokalisation und der Größe des Knorpelschadens ab. Ebenfalls sind möglicherweise vorliegende Begleitverletzungen (Kreuzband, Seitenband, Meniskusläsion) zu berücksichtigen.

\section{Konservative Therapie}

Finden sich lediglich Knorpelerweichungen (Chondromalazie I), werden diese nicht operativ angegangen. $\mathrm{Zu} \mathrm{Be}$ ginn der arthroskopischen Ära war es oft üblich, erweichte Knorpelareale zu entfernen, insbesondere wenn über femoropatellare Schmerzen geklagt wurde. Der erweichte Knorpel (Chondromalazie Grad I) wird immer funktionell ohne operative Knorpelmaßnahmen behandelt. Insbesondere gilt es den Patienten zu beruhigen. Sind Kinder und jugendliche Sportler betroffen, gilt dies auch für die Eltern.

Therapeutisch stehen bei verkürzter Muskulatur Dehnungsübungen im Vordergrund der Therapie. Bei beidseitigem Auftreten von femoropatellaren Schmerzen müssen auch Haltungsinsuffizienzen im Bereich Wirbelsäule und Fußfehlstellungen diagnostisch ausgeschlossen oder bestätigt werden.

Findet sich im MRT ein ausgedehntes Knochenödem (Bone Bruises), sollte eine mehrwöchige Schonung des Gelenks erfolgen. Bei ausgedehnten Befunden empfiehlt sich eine Teilbelastung (10-20 kg), damit sich das Knochenödem zurückbilden kann. Unterstützend können eine Sauerstofftherapie und Kalzitoninpräparate sein.

Beim Spitzensportler sollte nach 8-12 Wochen eine erneute MR-Tomografie erfolgen, um die Rückbildung des Knochenödems zu verifizieren. Entscheidend ist jedoch das klinische Bild. Hat sich das Knochenödem nur minimal zurückgebildet, der subjektive Befund jedoch deutlich gebessert, kann vorsichtig wieder mit der sportlichen Aktivität begonnen werden. Dieser Sportler ist jedoch sehr sorgfältig zu kontrollieren und $\mathrm{zu}$ instruieren (Gelenkschonung). Kommt es zu einer Schmerzzunahme, ist eine erneute MRT angezeigt, um eine Zunahme des Knochenödems auszuschließen. 

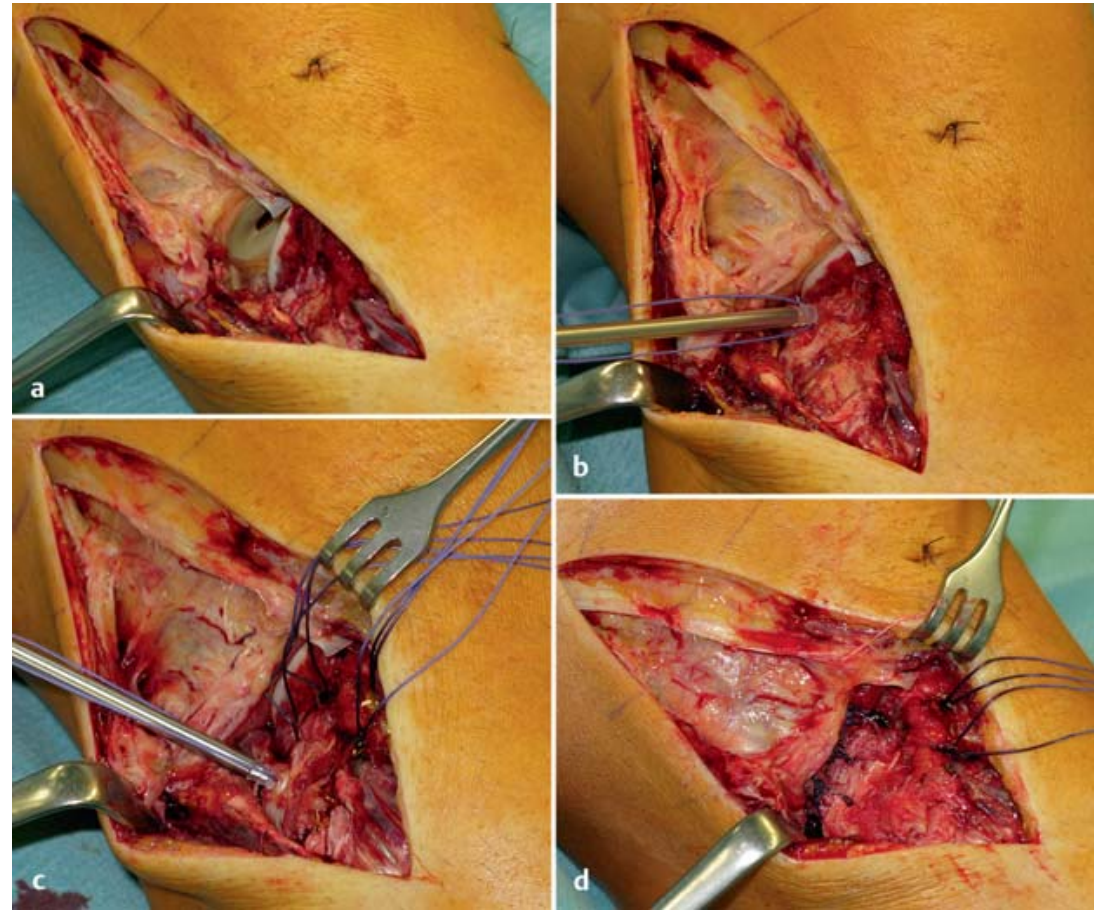

Abb. 75a bis d Anatomische Refixation einer komplexen lateralen und posterolateralen Instabilität (Abb. 74). Es findet sich eine komplette Ruptur des lateralen und posterolateralen Kapsel-Band-Apparates. Der Außenmeniskus ist komplett von der tibialen Insertion abgelöst (a). Setzen des ersten bioresorbierbaren Bioplugs (Fa. Karl Storz, Tuttlingen) zur Refixation des Meniskus und der lateralen Kapsel (b). Nachdem die Nähte gelegt sind, erfolgt das Setzen eines weiteren Bioplugs im Fibulaköpfchen zur Refixation des lateralen Seitenbands (c). Zustand nach Refixation der lateralen Kapsel und des Seitenbands. Der ruturierte Tractus iliotiialis wird noch mit den 2 in der anterolateralen Tibia platzierten Bioplugs anatomisch refixiert $(\mathbf{d})$.
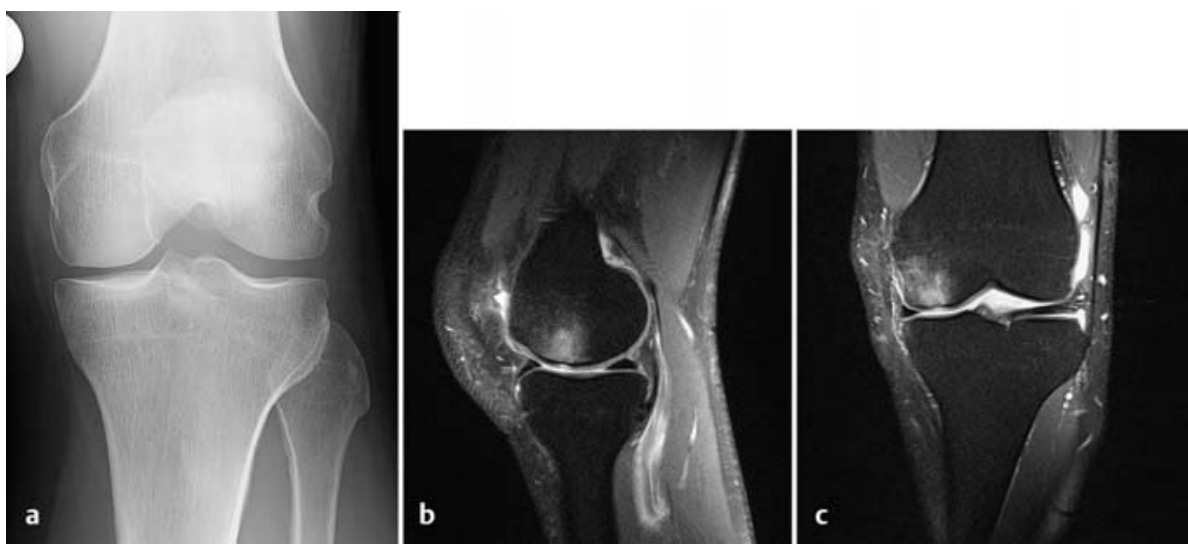

Abb. 76a bis c Knochenödem be 41-jährigem Läufer, der nach einem Trainingslauf über massive Schmerzen klagte. Unauffälliger Röntgenbefund (a). Im MRT zeigt sich ein Knochenödem im medialen Femurkondylus (b), das vorwiegend in der medialen Hälfte des Kondylus lokalisiert ist (c).
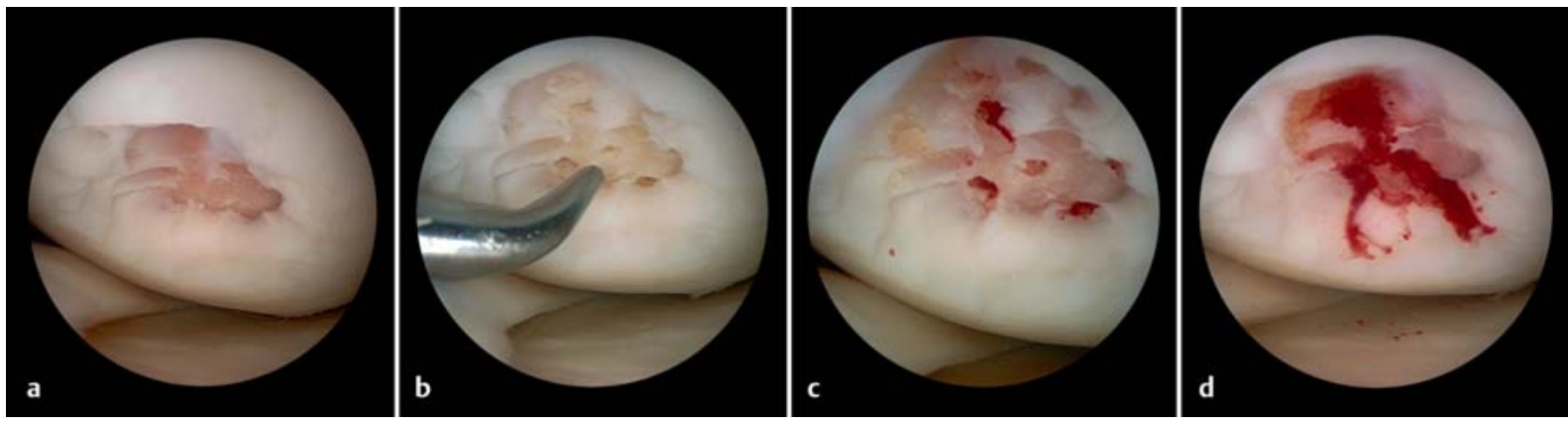

Abb. 77a bis d Microfracture-Technik bei Knorpelschaden am medialen Femurkondylus (a). Mit dem Mikrofrakturmeißel wird die Defektzone angemeißelt (b). Es treten zuerst kleine Fetttropfen, dann auch Blut aus (c). Wird der intraartikuläre Flüssigkeitsdruck reduziert, kommt es zur großflächigen Einblutung aus dem Knochen (d). 


\section{Operative Therapie}

Herausgebrochene Knorpelstücke und abstehende Knorpellappen werden entfernt, sofern eine Refixation nicht möglich oder sinnvoll ist. Im Allgemeinen ist die Refixation eines ausgebrochenen Knorpelstückes sinnvoll, wenn der umgebende Knorpel intakt ist, und das Ausmaß $10 \times 10 \mathrm{~mm}$ übersteigt. In Abhängigkeit der Lokalisation ist eine arthroskopische Refixation mit Fixationsstiften (z.B. Ethipins oder Polypins) möglich. Dies ist dann erfolgversprechend, wenn es sich um junge Patienten handelt ( $<20$ Jahre). Ist eine Refixation nicht möglich, wird das freiliegende Knochenareal mit einer Mikrofrakturierung zur Induktion von Ersatzknorpel behandelt (Abb. 77). Da in der Nachfolgezeit eine mehrwöchige Entlastung (6 - 8 Wochen) erforderlich ist, muss mit einer entsprechend langen Sportpause gerechnet werden.

Bei ausgedehnten Knorpelschäden $(>2 \times 1 \mathrm{~cm})$ muss abgewogen werden, ob eine autologe Chondrozytentransplantation angezeigt ist. Hier bieten sich verschiedene matrixgestützte Techniken an. Bezüglich der detaillierten Operationstechnik wird auf die verschiedenen Operationslehren verwiesen.

\section{Literatur}

${ }^{1}$ Adachi N, Ochi M, Uchio Y, Iwasa J, Ryoke K, Kuriwaka M. Reconstruction of the anterior cruciate ligament: single versus double bundle multistranded hamstring tendons. J Bone Joint Surg [Br] 2004; 86: 515-520

2 Aglietti P, Buzzi R, D'Andria S, Zaccherotti G. Patellofemoral problems after intraarticular anterior cruciate ligament reconstruction. Clin Orthop 1993; 288: 195-204

3 Aglietti P, Giron F, Cuomo P, Losco M, Mondanelli $N$. Single- and double-incision doublebundle ACL reconstruction. Clin Orthop Relat Res 2007; 454: 108-113

4 Aichroth P. Patel D, Zorilla P. The natural history and treatment of rupture of the anterior cruciate ligament in children and adolecents. J Bone Joint Surg [Br] 2002; 84: 38 - 41

${ }^{5}$ Arnold MP, Kooloos J, van Kampen A. Singleincision technique misses the anatomical femoral anterior cruciate ligament insertion: a cadaver study. Knee Surg Sports Traumatol Arthrosc 2001; 9: 194 - 199

${ }^{6}$ Barrack R, Bruckner J, Kneisl J, Inman W, Alexander $A$. The outcome of nonoperatively treated complete tears of the anterior cruciate ligament in active young adults. Clin Orthop 1990; 259: 192 - 199

7 Barrack R, Skinner H, Buckley S. Proprioception in the anterior cruciate insufficient knee. Am J Sports Med 1989; 17: 1-6

8 Bartlett R, Clatworthy M, Nguyen T. Graft selection in reconstruction of the anterior cruciate ligament. J Bone Joint Surg $[\mathrm{Br}] 2001$; 83: $625-634$

9 Buzzi R, Zaccherotti G, Giron F, Aglietti P. The relationship between the intercondylar roof and the tibial plateau with the knee in extension: relevance for tibial tunnel placement in anterior cruciate ligament reconstruction. Arthroscopy 1999; 15: 625-631

10 Casteleyn P, Handelberg F. Non-operative management of anterior cruciate ligament injuries in the general population. J Bone Joint Surg [Br] 1996; 78: 446-451

11 Castoldi F, Bonasia DE, Marmorotti A, Dettoni $F$, Rossi $R$. ACL reconstruction using Rigidfix femoral device via the anteromedial portal: a cadaver study to evaluate chondral lesions. Knee Surg Sports Traumatol Arthrosc 2008; 16: $275-278$

12 Cha PS, Brucker PU, West RV, Zelle BA, Yagi M, Kurosaka M, Fu FH. Arthroscopic doublebundle anterior cruciate ligament reconstruction: an anatomic approach. Arthroscopy 2005; 21: 1275

13 Chhabra A, Kline AJ, Nilles KM, Harner CD. Tunnel expansion after anterior cruciate ligament reconstruction with autogenous hamstrings: a comparison of the medial portal and transtibial techniques. Arthroscopy 2006; 22: 1107-1112

14 Corry I, Webb J, Clingeleffer A, Pinczewski L. Arthroscopic reconstruction of the anterior cruciate ligament: a comparison of patellar tendon autograft and four-strand hamstring tendon autograft. Am J Sports Med 1999; 27: $444-454$

${ }^{15}$ Daniel D, Malcom L, Losse G, Stone M, Sachs R, Burks $R$. Instrumented measurement of anterior laxity of the knee. J Bone Joint Surg [Am] 1985; 67: 720-725

16 Daniel DM, Stone M. Diagnosis of knee ligament injuries: tests and measurements of joint laxity. In Feagin JA, ed. The cruciate ligaments. Diagnosis and treatment of ligamentous injuries about the knee. Oxford: Churchill Livingstone; 1988: 287-300

17 Edwards T, Greene C, Baratta R, Zieske A, Willis $R$. The effect of placing a tensioned graft across open growth plates. A gross and histologic analysis. J Bone Joint Surg [Am] 2001; 83: $725-734$

18 Fanelli G, Edson C. Posterior cruciate ligament injurie in trauma patients - Part II. Arthroscopy 1995; 11: 526-529

19 Fauno $P$, Kaalund $S$. Tunnel widening after hamstring anterior cruciate ligament reconstruction is influenced by the type of graft fixation used: a prospective randomized study. Arthroscopy 2005; 21: $1337-1341$

20 Freedman K, D'Amato M, Nedeff D, Katz A, Bach B. Arthroscopic anterior cruciate ligament reconstruction: a metaanalysis comparing patellar tendon and hamstring tendon autografts. Am J Sports Med 2003; 31: $2-11$

21 Gilchrist J, Mandelbaum B, Meelacon H, Ryan GW, Silvers HJ, Griffin LY, Watanabe DS, Dick $R W$, Dvorak J. A randomized controlled trial to prevent noncontact anterior cruciate ligament injury in female collegiate soccer players. Am J Sports Med 2008; 36: 1476-1483

22 Hamner D, Brown C, Steiner $M$, Hecker A, Hayes $W$. Hamstring tendon grafts for reconstruction of the anterior cruciate ligament: biomechanical evaluation of the use of multiple strands and tensioning techniques. J Bone Joint Surg [Am] 1999; 81: 549-557

23 Harner C, Höher J. Evaluation and treatment of posterior cruciate ligament injuries. Am J Sports Med 1998; 26: 471-482

24 Hewett T, Noyes F, Lee M. Diagnosis of complete and partial posterior cruciate ligament ruptures: stress radiography compared with KT-1000 arthrometer and posterior drawer testing. Am J Sports Med 1997; 25: 648 - 655
${ }^{25}$ Höher J, Moller HD, Fu FH. Bone tunnel enlargement after anterior cruciate ligament reconstruction: fact or fiction? Knee Surg Sports Traumatol Arthrosc 1998; 6: 231 - 240

${ }^{26}$ Howell S, Taylors M. Failure of reconstruction of the anterior cruciate ligament due to impingement by the intercondylar roof. J Bone Joint Surg [Am] 1993; 75: 1044-1055

${ }^{27}$ Howell SM, Clark JA, Farley TE. A rationale for predicting anterior cruciate graft impingement by the intercondylar roof. A magnetic resonance imaging study. Am J Sports Med 1991; 19: 276 - 282

${ }^{28}$ Howell SM, Clark JA. Tibial tunnel placement in anterior cruciate ligament reconstructions and graft impingement. Clin Orthop Relat Res 1992; 283: 187- 195

${ }^{29}$ Howell SM. Principles for placing the tibial tunnel and avoiding roof impingement during reconstruction of a torn anterior cruciate ligament. Knee Surg Sports Traumatol Arthrosc 1998; 6: 49-55

${ }^{30}$ Hughston J, Andrews J, Cross M, Moschi A. Classification of knee ligament instabilities. Part I: the medial compartment and cruciate ligaments. J Bone Joint Surg [Am] 1976; 58: $159-172$

${ }^{31}$ Hughston J, Eilers A. The role of the posterior oblique ligament in repairs of acute medial collateral ligament tears of the knee. J Bone Joint Surg [Am] 1973; 55: 923 - 940

32 Hunt $P$, Rehm $O$, Weiler A. Soft tissue graft interference fit fixation: observations on graft insertion site healing and tunnel remodelling 2 years after ACL reconstruction in sheep. Knee Surg Sports Traumatol Arthrosc 2006; 14: 1245-1251

${ }^{33}$ Hurd WJ, Axe MJ, Snyder-Mackler L. A 10 years prospective trial of a patient management algorithm and screening examination for highly active individuals with anterior cruciate ligament injury. Part 1 - outcomes. Am J Sports Med 2008; 36: 40-47

34 Hurd WJ, Axe MJ, Snyder-Mackler L. A 10 years prospective trial of a patient management algorithm and screening examination for highly active individuals with anterior cruciate ligament injury. Part 2 determinations of dynamic knee stability. Am J Sports Med 2008: 36: $48-56$

35 Iorio R, Vadala A, Argento G, Di Sanzo V, Ferretti $A$. Bone tunnel enlargement after ACL reconstruction using autologous hamstring tendons: a CT study. Int Orthop 2007; 31: 49-55

${ }^{36}$ Jackson DW, Gasser SI. Tibial tunnel placement in ACL reconstruction. Arthroscopy 1994; 10: 124-131

37 Jacobsen K. Gonylaxometry: stress radiographic measurement of passive stability in the knee joints of normal subjects and patients with ligament injuries. Accuracy and range of application. Acta Orthop Scand (Suppl) 1981; 194: 1-263

38 Jacobsen K. Stress radiographical measurement of anterior-posterior, medial and lateral stability of the knee joint. Acta Orthop Scand 1976; 47: 335 - 341

39 Jarvela T. Double-bundle versus single-bundle anterior cruciate ligament reconstruction: a prospective, randomize clinical study. Knee Surg Sports Traumatol Arthrosc 2007; 15: $500-507$

40 Johnson D, Laboureau J. Cruciate ligament reconstruction with synthetics. 2001; 14: 189 214

${ }^{41}$ Kannus P. Long-term results of conservatively treated medial collateral ligament injuries of the knee. Clin Orthop 1988; 226 : $103-112$ 
42 Kartus J, Magnusson L, Stener S, Brandsson, Eriksson B, Karlsson J. Complications following arthroscopic anterior cruciate ligament reconstruction - a 2-5 year follow-up of 604 patients with special emphasis on anterior knee pain. Knee Surg Sports Traumatol Arthrosc 1999; 7: 2-8

${ }^{43}$ Kartus J, Stener S, Lindahl S, Engstrom B, Eriksson B, Karlsson J. Factors affecting donor-site morbidity after anterior cruciate ligament reconstruction using bone-patellar tendonbone autografts. Knee Surg Sports Traumatol Arthrosc 1997; 5: $222-228$

${ }^{44}$ Kennedy J, Fowler P. Medial and anterior instability of the knee. J Bone Joint Surg [Am] 1971; 53: $1257-1270$

45 Kessler MA, Behrend H, Hnez S, Stutz G, Rukavina A, Kuster MS. Function, osteoarthritis and activity after ACL rupture: 11 years follow-up results of conservative versus reconstructive treatment. Knee Surg Sports Traumatol Arthrosc 2008; 16: 442 - 448

${ }^{46}$ Kobayashi M, Nakagawa Y, Suzuki T, Okudaira $S$, Nakamura T. A retrospective review of bone tunnel enlargement after anterior cruciate ligament reconstruction with hamstring tendons fixed with a metal round cannulated interference screw in the femur. Arthroscopy 2006; 22: $1093-1099$

47 Larson $R$, Metcalf $M$. Posterior cruciate ligament reconstruction: associated extra-articular procedures medial and lateral. Tech $\mathrm{Or}$ thop 2001; 16: 148 - 156

${ }^{48}$ Loh J, Fukuda Y, Tsuda E, Steadman J, Fu F, Woo $S$. Knee stability and graft function following anterior cruciate ligament reconstruction: comparison between 11 o'clock and 10 o'clock femoral tunnel placement. Arthroscopy 2003; 19: 297-304

${ }^{49}$ Malek MM, DeLuca JV, Verch DL, Kunkle KL. Arthroscopically assisted ACL reconstruction using central third patellar tendon autograft with press fit femoral fixation. Instr Course Lect 1996; 45: 287-295

${ }^{50}$ Markolf K, Slauterbeck J, Armstrong K, Shapiro $M$, Finerman G. Effects of combined knee loadings on posterior cruciate ligament force generation. J Orthop Res 1996; 14: 633-637

51 Mohtadi NGH, Webster-Bogaert S, Fowler PJ. Limitation of motion following anterior cruciate ligament reconstruction. Am J Sports Med 1991; 19: 620-625

52 Müller W. Das Knie. Form, Funktion und ligamentäre Wiederherstellungschirurgie. Berlin: Springer; 1982

53 Muneta T, Yamamoto H, Ishibashi T, Asahina S Murakami S, Furuya K. The effects of tibial tunnel placement and roofplasty on reconstructed anterior cruciate ligament knees. Arthroscopy 1995; 11: 57-62

${ }^{54}$ Myer GD, Ford KR, Paterno MV Nick TG, Hewett TE. The effects of gerneralized joint laxity on the risk of anterior cruciate ligament injury in young female athletes. Am J Sports Med 2008; 36: 1073-1080

${ }^{55}$ Neyret P, Donell S, DeJour D, DeJour H. Partial meniscectomy and anterior cruciate ligament rupture in soccer players. A study with a minimum 20-year follow-up. Am J Sports Med 1993; 21: 455-460

${ }^{56}$ Nottage W, Matsuura P. Management of complete traumatic anterior cruciate ligament tears in the skeletaly immature patient: current concepts and review of the literature. Arthroscopy 1994; 10: 569-573

57 Noyes F, Mangine R, Grood E. Biomechanical analysis of human ligament grafts used in knee-ligament repairs and reconstruction. J Bone Joint Surg [Am] 1984; 66: 344-352
58 Pässler H, März S. The radological Lachman test - a simple and sure method for detection of damage to the cruciate ligament. Unfallchirurgie 1986; 12: 295 - 300

59 Paul J, Spindler K, Andrish J, Parker R, Secic M, Bergfeld J. Jumping versus nonjumping anterior cruciate ligament injuries - a comparison of pathology. Clin J Sport Med 2003; 13: $1-5$

60 Petersen W, Tillmann B. Structure and vascularization of the cruciate ligaments of the human knee joint. Anat Embryol (Berl) 1999; 200: 325-334

${ }^{61}$ Petersen W, Tillmann B. Anatomy and function of the anterior cruciate ligament. Orthopäde 2002; 31: 710-718

62 Petersen W, Tretow H, Weimann A, Herbort M, Fu FH, Raschke M, Zantop T. Biomechanical evaluation of two techniques for doublebundle anterior cruciate ligament reconstruction: one tibial tunnel versus two tibial tunnels. Am J Sports Med 2007; 35: 228 234

63 Petersen $W$, Zantop T. Technik der Doppelbündelrekonstruktion. Arthroskopie 2007; 20: $132-138$

64 Race A, Amis A. The mechanical properties of the two bundles of the human posterior cruciate ligament. J Biomech 1994; 27: 13-24

65 Race A, Amis A. PCL reconstruction: in vitro biomechanical evaluation of isometric versus single and double bundle anatomic grafts. J Bone Joint Surg [Br] 1998; 80: 173 179

${ }^{66}$ Richmond JC, Manseau CJ, Patz R, McConville $O$. Anterior cruciate reconstruction using a Dacron ligament prosthesis. A long-term study. Am J Sports Med 1992; 20: 24-28

67 Romano VM, Graf BK, Keene JS, Lange RH. Anterior cruciate ligament reconstruction. The effect of tibial tunnel placement on the range of motion. Am J Sports Med 1993; 21: $415-$ 418

68 Rosenberg T, Paulos L, Parker R, Coward D, Scott $S$. The fourty-five-degree posteroanterior flexion weight-bearing radiograph of the knee. J Bone Joint Surg [Am] 1988; 70: $1479-1483$

${ }^{69}$ Schulz M, Russe K, Weiler A, Eichhorn H, Strobel $M$. Epidemiology of posterior cruciate ligament injuries. Arch Orthop Trauma Surg 2003; 123: 186-191

70 Segawa H, Omori G, Koga Y, Kameo T, Iida S, Tanaka M. Rotational muscle strength of the limb after anterior cruciate ligament reconstruction using semitendinosus and gracilis tendon. Arthroscopy 2002; 18: 177 - 182

71 Shelbourne KD, Nitz P. Accelerated rehabilitation after anterior cruciate ligament reconstruction. Am J Sports Med 1990; 18: $292-$ 299

72 Shelbourne KD, Rettig AC, McCarrol JR, Bisesi MA, Vogel A, Kuhn D. Functional ability in athletes with an anterior cruciate ligament deficiency. Am J Sports Med 1987; 15: 391 392

73 Shelbourne KD, Wilckens JH. Intraarticular anterior cruciate ligament reconstruction in the symptomatic arthritic knee. Am J Sports Med 1993; 21: 685-689

74 Shelbourne KD, Wilckens JH, Mollabashy A, DeCarlo M. Arthrofibrosis in acute anterior cruciate ligament reconstruction. The effect of timing of reconstruction and rehabilitation. Am J Sports Med 1991; 19: 332-336

75 Shoemaker B, Markolf K. In vivo rotatory knee stability. J Bone Joint Surg [Am] 1982; 64: 208-216
${ }^{76}$ Singhatat W, Lawhorn KW, Howell SM, Hull $M L$. How four weeks of implantation affect the strength ans stiffness of a tendon graft in a bone tunnel. Am J Sports Med 2002; 30: 506-513

77 Skyhar M, Warren R, Ortiz G, Schwartz E, Otis $J$. The effects of the posterior cruciate ligament and posterolateral complex on the articular contact pressures within the knee. J Bone Joint Surg [Am] 1993; 75: 694 -699

78 Sommer C, Friederich NF, Muller W. Improperly placed anterior cruciate ligament grafts: correlation between radiological parameters and clinical results. Knee Surg Sports Traumatol Arthrosc 2000; 8: 207-213

${ }^{79}$ Stähelin A, Weiler A. All-inside anterior cruciate ligament reconstruction using semitendinosus tendon and soft threaded biodegradable interference screw fixation. Arthroscopy 1997; 13: 773-779

${ }^{80}$ Streich NA, Friedrich K, Gotterbarn T, Schmitt $H$. Reconstruction of the ACL with a semitendinosus tendon graft: a prospective randomized single blinded comparison of double-bundle versus single bundle techniques in male athletes. Knee Surg Sports Traumatol Arthrosc 2008; 16: 232 -238

${ }^{81}$ Strobel M, Stedtfeld $H$. Gehaltene Röntgenaufnahmen des Kniegelenkes. Unfallchirurg 1986; 89: 272 - 279

82 Strobel M. Diagnostik des Kniegelenkes. 3. Aufl. Berlin, Heidelberg, New York: Springer Verlag; 1995

83 Strobel M. Arthroskopische Chirurgie. Berlin, Heidelberg, New York, Tokyo: Springer; 1998

${ }^{84}$ Strobel M, Weiler A, Eichhorn H. Diagnosis and treatment of acute and chronic posterior cruciate ligament insufficiency. Chirurg 2000; 71: 1066 - 1081

85 Strobel M, Weiler A. Pitfalls and pearls for the management of the posterior cruciate ligament deficient knee. Tech Orthop 2001; 16: 167-194

${ }^{86}$ Strobel M, Weiler A, Schulz M, Russe K, Eichhorn $H$. Fixed posterior cruciate subluxation in posterior cruciate ligament deficient knees. Am J Sports Med 2002; 30: 32 - 38

87 Strobel M, Weiler A, Schulz M, Russe K, Eichhorn $H$. Arthroscopic evaluation of articular cartilage lesions in posterior cruciate ligament deficient knees. Arthroscopy 2003; 19: $262-268$

88 Strobel M, Weiler A. Hinteres Kreuzband Anatomie - Diagnostik - Operationstechnik. Tuttlingen: Endopress-Verlag; 2008

89 Tashman S, Collon D, Anderson K, Kolowich $P$, Anderst $W$. Abnormal rotational knee motion during running after anterior cruciate ligament reconstruction. Am J Sports Med 2004; 32: 975-983

90 Tomihara T, Ohashi H, Yo H. Comparison of direct and indirect interference screw fixation for tendon grafts in rabbits. Knee Surg Sports Traumatol Arthrosc 2007; 15: 26-30

${ }^{91}$ Tsuda E, Fukuda Y, Loh J, Debski R, Fu F, Woo S. The effect of soft-tissue graft fixation in anterior cruciate ligament reconstruction on graft-tunnel motion under anterior tibial loading. Arthroscopy 2002; 18: 960-967

92 Viola R, Sterett W, Newfield D, Steadman J, Torry $M$. Internal and external tibial rotation strength after anterior cruciate ligament reconstruction using ipsilateral semidentinosus and gracilis tendon autograft. Am J Sports Med 2000; 28: 552-555

93 Weiler A, Hoffmann R, Bail H, Rehm O, Südkamp NP. Tendon healing in a bone tunnel Part II. Histological analysis after biodegradable interference fit fixation in a model of anterior cruciate ligament reconstruction in sheep. Arthroscopy 2002; 18: 124-135 
94 Weiler A, Scheffler S, Höher J. Transplantatauswahl für den primären Ersatz des vorderen Kreuzbandes. Orthopäde 2002; 31 : $731-740$

95 Weimann A, Zantop T, Herbort M, Strobel M, Petersen $W$. Initial fixation strength of a hybrid technique for femoral ACL graft fixation. Knee Surg Sports Traumatol Arthrosc 2006; 14: $1122-1129$

96 Wilson T, Kantaras A, Atay A, Johnson DL. Tunnel enlargement after anterior cruciate ligament surgery. Am J Sports Med 2004; 32 $543-549$

97 Yagi M, Kuroda R, Nagamune K, Yoshiya S, Kurosaka M. Double-bundle ACL reconstruction can improve rotational stability. Clin Orthop Relat Res 2007; 454: 100-107

98 Yamamoto Y, Hsu WH, Woo SL, Van Scyoc AH, Takakura Y, Debski RE. Knee stability and graft function after anterior cruciate liga- ment reconstruction: a comparison of a lateral an anatomical femoral tunnel placement. Am J Sports Med 2004; 32: 1825 1832

99 Yasuda K, Kondo E, Ichiyama H, Kitamura N, Tanabe Y, Tohyama H, Minami A. Anatomic reconstruction of the anteromedial and posterolateral bundles of the anterior cruciate ligament using hamstring tendon grafts. Arthroscopy 2004; 20: 1015 - 1025

${ }^{100}$ Yasuda K, Kondo E, Ichiyama H, Tanabe Y, Tohyama $H$. Clinical evaluation of anatomic double-bundle anterior cruciate ligament reconstruction procedure using hamstring tendon grafts: comparisons among 3 different procedures. Arthroscopy 2006; 22: 240-251

${ }^{101}$ Yasuda K, Tsujino J, Ohkoshi Y, Tanabe Y, Kaneda K. Graft site morbidity with autogenous semitendinosus and gracilis tendons. Am J Sports Med 1995; 23: $706-714$
${ }^{102}$ Zantop T, Herbort M, Raschke MJ, Fu FH, Petersen $W$. The role of the anteromedial and posterolateral bundles of the anterior cruciate ligament in anterior tibial translation and internal rotation. Am J Sports Med 2007; 35: $223-227$

\section{Prof. Dr. med. Michael J. Strobel}

sporthopaedicum Straubing Orthopädisch-Chirurgische Gemeinschaftspraxis

Bahnhofplatz 8

94315 Straubing 